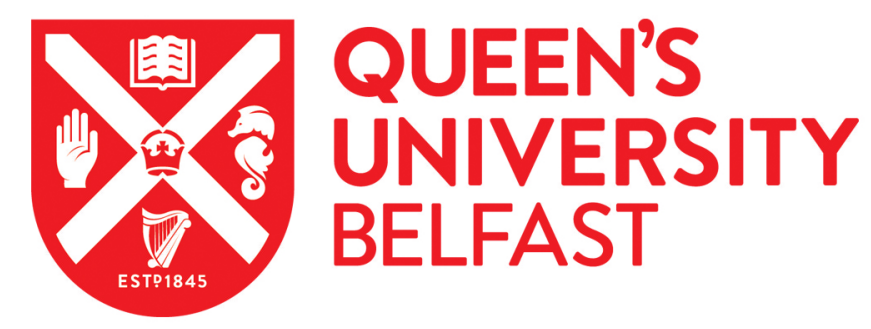

\title{
Heterogeneity in Early Life Investments: A Longitudinal Analysis of Children's Time Use
}

Rokicki, S., \& McGovern, M. (2019). Heterogeneity in Early Life Investments: A Longitudinal Analysis of Children's Time Use. Review of income and wealth.

\section{Published in:}

Review of income and wealth

\section{Document Version:}

Peer reviewed version

Queen's University Belfast - Research Portal:

Link to publication record in Queen's University Belfast Research Portal

\section{Publisher rights}

(c) 2019 International Association for Research in Income and Wealth.

This work is made available online in accordance with the publisher's policies. Please refer to any applicable terms of use of the publisher.

\section{General rights}

Copyright for the publications made accessible via the Queen's University Belfast Research Portal is retained by the author(s) and / or other copyright owners and it is a condition of accessing these publications that users recognise and abide by the legal requirements associated with these rights.

Take down policy

The Research Portal is Queen's institutional repository that provides access to Queen's research output. Every effort has been made to ensure that content in the Research Portal does not infringe any person's rights, or applicable UK laws. If you discover content in the Research Portal that you believe breaches copyright or violates any law, please contact openaccess@qub.ac.uk. 
Heterogeneity in Early Life Investments: A Longitudinal Analysis of Children's Time Use*

Slawa Rokicki† and Mark E. McGovern

June 2019

\begin{abstract}
We examine socioeconomic heterogeneity in children's time use using diary data from two waves of a nationally representative longitudinal cohort study in Ireland. Children from disadvantaged households spend significantly less time reading, doing homework, and engaging in sport than their counterparts, and more time engaging in unstructured play. Though most gaps are relatively small at age 9, they widen considerably by age 13 . This pattern is similar for girls and boys. Parental education appears to be a more important factor in family investment decisions about children's time use than household income. Given the important role of extra-curricular activities in promoting cognitive and non-cognitive skill development, the systematic differences in children's time use we document in this paper may contribute to cumulative disadvantage and widening skill gaps through adolescence and into adulthood.
\end{abstract}

Keywords: Time Use, Inequality, Socioeconomic Status, Early Life Conditions; Skill Development

JEL Classification: I30, J10, J22

\footnotetext{
* Forthcoming in Review of Income and Wealth.

${ }^{+}$Geary Institute for Public Policy, University College Dublin.

‡ Queen's University Belfast.
} 


\section{Introduction}

How children spend their time has important implications for their cognitive and non-cognitive development. Activities pursued in one context, such as participating in extra-curricular engagements, contribute to the development of competencies in another, such as in school or the labour market. Activities during after-school hours in particular are an important contributor to children's developmental trajectories (Posner and Vandell, 1999). Sports and other prosocial activities promote growth and positive development by creating opportunities for belonging, cooperating with others, and skill building (Fredricks and Eccles, 2006). Activities such as reading and doing homework directly contribute to learning (Hofferth and Sandberg, 2001), as well as provide indirect learning opportunities such as development of attention, self-regulation, and self-esteem (Posner and Vandell, 1999). Free play activities may also contribute to developing initiative and problem solving skills (Hofferth and Sandberg, 2001; Burdette and Whitaker, 2005).

Moreover, there is broad evidence linking involvement in extra-curricular activities to a wide range of health, social, and labour outcomes in adulthood (Heckman, 2008). For example, participation in sports is associated with higher educational attainment, labor force participation, and wages (Barron, Ewing and Waddell, 2000; Lipscomb, 2007; Pfeifer and Cornelißen, 2010; Stevenson, 2010). Educational activities at young ages, such as reading and computer time, are associated with increased test scores at later ages (Fiorini, 2010; Fiorini and Keane, 2014). Participation in health-promoting activities and physical exercise in childhood may impact on adult health directly through reducing the risk of overweight and obesity (DeMattia, Lemont and Meurer, 2007) and indirectly by promoting transmission of these healthy behaviours and routines into later life (Perkins et al., 2004; Telama et al., 2005). Poor health in childhood, including overweight and obesity, has been found to predict poor health in adulthood as well as affect education and labour outcomes (Gortmaker et al., 1993; Black, Devereux and Salvanes, 2007; Smith, 2009; Delaney, McGovern and Smith, 2011).

If time use differences are systematically associated with family background, differences in children's time use, particularly during non-school hours, are a potential contributor to the emergence and persistence of inequalities in cognitive and non-cognitive skills. This hypothesis is supported by evidence from a wide range of contexts showing inequalities in non-cognitive skills by socioeconomic status (SES) (Heckman, 2008). Moreover, in the US there are diverging trends in children's time spent in skill-promoting activities by parental education (Ramey and Ramey, 2010; Altintas, 2016; Putnam, 2016). Given that half of the inequality in lifetime earnings has been argued to be due to factors determined by age 18 (Heckman, 2008), the policy implications of inequality in children's time use are potentially important.

Though there exists a wide range of literature describing heterogeneity in parental time spent with children across family background (see, for example, Gustafsson and Kjulin 1994; Hallberg and Klevmarken 2003; Sayer et al. 2004; Guryan et al. 2008; Kalil et al. 2012; Fiorini and Keane 2014), there is very little research describing heterogeneity in children's own time use. While parental time spent with children is an important input in its own right, it does not capture an important component of children's activities, that is, what children do outside of time spent with parents.

In this paper, we examine socioeconomic differences in children's time use in a longitudinal cohort in Ireland. We aim to answer the following research questions: (1) what is the extent of inequality in children's time use by family socioeconomic background, and (2) how do these socioeconomic differences in time use change over the period from middle childhood to early adolescence? To guide our analysis, we apply a theoretical framework based on a human capital production, detailed below, that classifies time use into investment activities (those activities which the literature has demonstrated are important for human capital acquisition and are associated with adult outcomes, i.e. reading, sports, and homework), and leisure activities (other activities for which the literature is less clear about their long run beneficial impacts). 
We make a number of contributions to the literature. First, we use detailed time diary data from a nationally representative longitudinal panel of school children. Time diary data is more accurate than data derived from stylized survey questions on overall time spent in a particular activity (Hofferth and Sandberg, 2001; Kan and Pudney, 2008). These data also capture all of a child's activities during a day, instead of focusing on a few particular categories. Thus, we are able to examine not only socioeconomic differences in time spent in specific activities, but also which activities are substituted for in their place as children age. Second, we examine the association between socioeconomic background and time use on both the extensive margin (participation in activities) and the intensive margin (length of participation). Third, we apply concentration curves to quantify the extent of income-related inequality in time use. Fourth, we use the longitudinal nature of our data to analyze trajectories in time use as children age. Finally, we use fixed effects models to account for time-invariant omitted factors common to children and families. To the best of our knowledge, this is the first paper to analyze longitudinal changes in time use among children in this way.

The rest of the paper is as follows: Section 2 provides a theoretical framework for understanding average differences in children's time use and why they are expected to grow as children age. Section 3 discusses the data, descriptive statistics, and our estimation strategy. Section 4 presents our results and Section 5 discusses the findings.

\section{Theoretical Background}

From an economics perspective, children's time use allocation can be viewed as the realisation of parents' and children's decisions about when and where to invest in human capital acquisition (Leibowitz, 1974; Haveman and Wolfe, 1995). Under a production function framework for human capital, investment decisions regarding the allocation of resources to inputs and children's time use will be based on parents aiming to maximize life cycle returns to them and their children (Becker and Tomes, 1986). Given these investments, children then also make choices to maximize their own welfare as they gain agency (Haveman and Wolfe, 1995). This framework can provide an insight into potential explanations for heterogeneous patterns of children's time use across families and across time as children age. In what follows we discuss the main features of the model, while a more formal representation is presented in Section A1 in the Appendix.

\subsection{Previous Evidence on Socioeconomic Differences in Average Time Use}

The first main feature of the framework we consider is that a variety of factors may contribute to differences in children's time use across levels of SES. We expect to see differences in children's time use by household income if some families cannot afford the costs associated with various activities and they are credit-constrained, thereby limiting parental investment decisions. Children from financially disadvantaged families are less likely to have access to material and cultural resources from infancy to adolescence (Bradley and Corwyn, 2002). Evidence suggests that disparity in parental resources by family background has been widening since the second demographic transition in the 1960s (McLanahan, 2004). For example, the direct costs of extra-curricular activity participation are estimated at $10 \%$ of annual income for a family of two children in the bottom income quintile in the US (Putnam, 2016). Parental time spent with children has been found to be positively associated with income (SousaPoza et al. 2001, Kalenkoski et al. 2009), suggesting that at least some of the difference in children's time use are due to resource constraints.

Costs associated with activities may not necessarily be monetary and may also depend on geographic location, proximity to amenities, and the opportunity cost. For example, high-income mothers are more likely to have flexible work schedules and spouses who are more involved in child rearing (Heckman, 2008; Kalil et al., 2012), while low-income parents are more likely to have inflexible and atypical (late, rotating, or weekend) work schedules; in the US this disparity has been increasing since the 1970s (Hamermesh, 2002). Evidence shows that for mothers without a college degree, maternal employment significantly reduces total time spent with children, while for highly educated mothers there is no correlation, suggesting that less-educated women have increased barriers to balancing work and family 
life (Hsin and Felfe, 2014). Other research has found that while number of hours worked generally exerts a negative effect on parental time with children, the negative impact of hours worked in the evening (between 6pm and 10pm) is twice as large as daytime work hours (Rapoport and Le Bourdais, 2008). Other potential costs include mother's marital status, as single mothers may have less available time to organize and manage their children's activities (Hofferth and Sandberg, 2001; Burton and Phipps, 2007; Fox et al., 2013). A study using the American Time Use Survey found that among teenagers aged 15-17, those in households with single mothers spend more time in unsupervised activities (Wight et al., 2009).

Neighbourhood characteristics may also influence parental decisions about children's time investments (Doyle, McEntee and McNamara, 2012; Kling, Liebman and Katz, 2007). For example, in a study of SES disparities in physical activity and screen time, Tandon et al. (2012) found that household rules around outdoor play are more restrictive in lower SES households while children's average screen time is higher. Their findings suggested that low SES parents have greater concerns about their neighbourhood's safety and have less access to alternative activities, which makes indoor screen-based diversions more appealing.

In addition, if initial endowments vary by SES as the literature supports (Currie, 2011), and the productivity of investments depends on these initial endowments, then we would also expect SES differences in children's time use to emerge because investment activities are more productive for higher SES families. Factors such as birth weight are strongly patterned by SES (Boardman et al., 2002; Hsin, 2012; McGovern, 2013). Although it can be difficult to separate out initial ability from parental responses as children age, it has been well established that gaps in cognitive ability appear very early in life (Heckman and Masterov, 2007). As a result, families may form differing beliefs about later life returns to investments informed by initial endowments (Kalil et al., 2012). This can manifest itself in the form of theories of parenting and may differ across SES. Highly educated parents may tend to "concertedly cultivate" their children's development due to beliefs that this will maximize their children's future opportunities. For example, they may organize and monitor children's after-school and weekend activities, use cognitively stimulating parenting practices more frequently, and leverage their social capital to advocate for their children in school (Harding, Morris and Hughes, 2015; Kalil et al., 2012; Lareau, 2003). Conversely, lower educated parents may tend to follow the parenting model of "accomplishment of natural growth", which encourages children to be independent and learn to make their own decisions in an effort to build their resilience and resourcefulness (Lareau, 2003). Kalil et al. (2012) show that compared to less educated mothers, more educated mothers invest more time in basic care and play with children under 6 years of age, and more time in management of activities (such as scheduling and monitoring extracurricular activities) for children aged 6 to 13. A wide range of evidence from the US and Europe finds that highly educated parents spend more time engaging with their children and monitoring their activities than do less educated parents (Sayer et al., 2004; Bianchi, Robinson and Milke, 2006; Guryan et al., 2008; Gimenez-Nadal and Molina, 2013). The fact that this trend is similar across countries despite substantial cross-national variation in levels of economic support and services for families suggests that better educated parents may have different parental values and behaviours than less educated parents (Sayer et al., 2004). Finally, in the UK, Delaney and Doyle (2012) present evidence that time preferences differ across SES, as measured by traits such as hyperactivity, impulsivity and persistence, and that they are transmitted through parents' non-cognitive skills such as self-esteem and attachment, as well as through parental time investments such as time spent reading to and teaching the child.

\subsection{Previous Evidence on Widening Differences in Time Use}

The second main feature of the framework we consider is that gaps in children's time use can be expected to widen over time for certain activities. From an economics perspective, increasing SES gaps in time use as children age may be explained by the presence of self-productivity, whereby skills gained at earlier ages promote skill acquisition in the future, and cross-complementarities, whereby prior time investments raise the returns to later investments. Due to these features, older children who received time inputs from parents when they were younger benefit more from investments in later periods. In 
addition, children who have greater skills at younger ages will be better able to develop new skills at older ages.

These insights from the economics literature can be combined with evidence from other disciplines. For example, one feature which is absent from a framework in which parents are the main decision-makers is the agency of the child and their capacity to decide about the activities on which to spend their time (Haveman and Wolfe, 1995). Children have increasing input into decisions about their time use as they age. One way to consider the implications of this is in terms of the self-productivity of skills. Children are likely to choose activities for which they feel they have an aptitude, or in other words, activities for which the entry costs are not too high. Additionally, social networks and peer groups can facilitate engagement in activities such as sports and we can view a lack of a peer group in terms of increasing the cost of engaging in the activity. These may also widen over time as children transition from familybased activities to school or friend-group based activities which rely on cooperation and support within peer groups. Finally, we can consider the literature on cumulative disadvantage. In sociology it is recognised that initial disparities can widen over time as different types of disadvantages combine to amplify initial inequalities in skill outcomes (DiPrete and Eirich, 2006). In terms of the model we describe above, this can be viewed as a virtuous cycle of parental and child investments whereby initial investments raise the return to later inputs.

\subsection{Research Hypotheses}

We apply this framework to consider two predictions about the association between socioeconomic background and children's time use, which we can test using our data.

Hypothesis 1: There are socioeconomic differences in children's average time use.

Based on the theoretical framework and existing evidence outlined in section 2.1, we hypothesize that there are differences in the types of activities in which children engage and the length of time spent in these activities across socioeconomic background.

Hypothesis 2: Socioeconomic differences in children's time use widen as children age.

Based on the theoretical framework and existing evidence outlined in section 2.2, we hypothesize that SES differences in time spent in investment activities (such as reading, sports, and homework) will grow wider as children age. As we describe above, self-productivity and cross-complementarities imply widening gaps in these activities as children age because they are path-dependent, relying on the development of skills in previous periods. To account for any relative increased time spent in these investment activities, children would need to decrease relative time spent in other domains, in particular, leisure activities. Therefore, in groups for whom relative time spent in investment activities increases over time, we expect a relative decrease in at least one leisure activity, and vice versa.

For both hypotheses, a variety of factors may contribute to explain observed differences in time use, including resources, initial endowments, and alternative beliefs about the return on investments. In this paper, we use mother's education and household income as measures of SES in order to examine possible mechanisms - in particular to establish the extent to which time use differences are due to resource constraints as opposed to differing beliefs about investments.

\section{Data and Methods}

\subsection{Data}

We use data from the Growing Up in Ireland (GUI) survey, a nationally representative longitudinal study of two cohorts (one of infants and one of children). We use the children's cohort, which first recruited and interviewed 8,568 nine-year-olds and their families in 2007/2008. A two-stage design was 
adopted that initially sampled primary schools and, subsequently, children within those schools. The second round of interviews occurred 4 years later, when 7,535 participants were successfully contacted at age 13 .

Both waves of the GUI child cohort included a Time Use Diary (TUD), which recorded details on the activities of participants over a 24-hour period, dividing the day and night into 15-minute intervals. In the first wave, parents were asked to complete the diary with their children (if possible); at the second wave, the 13-year-old children were asked to complete the diary with the help of their parents (if necessary).

There were 22 pre-coded activities in wave 1 and up to five activities could be recorded concurrently. In wave 2, there were 21 pre-coded activities (and 4 spaces for specifying 'other' activities), and up to 3 activities could be recorded concurrently. Respondents were not asked to prioritize concurrent activities. However, only $1 \%$ of time slots had concurrent activities (3\% of the after-school hours of $2 \mathrm{pm}$ to $9 \mathrm{pm}$ ); therefore, we only use data on the first activity recorded. The lists of possible activities were not the same across the two waves, therefore in order to compare time use at ages 9 and 13 we consolidate the activities into 12 categories: sleeping, personal care (which includes eating, traveling, and personal care), school, homework, sport/exercise, playing/unstructured time, hobbies, media (which includes watching TV and videos, using the computer/internet, using phones, using social media, and listening to music), reading for pleasure, housework, family time (which includes shopping trips and outings), and other. A summary of these categories is shown in Table A1 in the Appendix.

Not all respondents completed the TUD: in wave 1, 6,228 (72.6\%) returned usable diaries and in wave 2 the corresponding number was 5,023 (67\% of whom had also completed the TUD in wave 1). Households were more likely to reply to the TUD survey if the primary caregiver (usually the mother) was older, not employed, more educated, and owned their home. To adjust for this, survey weights were provided to ensure that the sub-sample of TUD respondents remained nationally representative (Quail and Williams, 2013; Quail and Williams, 2015). Table 1 demonstrates that the weighted characteristics of the TUD respondents matches those of the main sample at each wave. We use these weights as part of all our descriptive analyses. Throughout the paper, we define a child's mother as a female parent or step-parent living in the household, regardless of marital or biological status. We also drop children whose mothers are not found in the household (1.5\% of all observations).

In the Irish education system children undertake 6 years of primary school. Currently all children must then complete at least 3 years of junior cycle secondary education (after which there is a national state exam), followed by either 2 or 3 additional years of senior cycle secondary education (depending on whether they undertake a "transition year" which is optional in some schools), at the end of which there is a second state state examination (the leaving certificate or related vocational qualifications) which forms the basis for university admission. In wave 1 of the GUI survey, cohort members were attending primary school, while in wave 2 , cohort members had transitioned to the junior cycle of secondary school.

As with the main family-based survey interviews, TUD data was collected throughout the year from August 2007 to July 2008 for wave 1, and from August 2011 to April 2012 for wave 2. Respondents were instructed as to what day of the week they were to complete the diary so as to distribute respondent days across the week. Respondents indicated if they completed the diary at the end of the diary day, the day after the diary day, or another day. They also indicated whether the diary day was during the school term or out of term, and what "type" of day it was (ordinary/school day, holiday, etc). Information on when and how the TUD was completed is provided in Table A2 in the appendix. We adjust for these diary variables in our analyses. In order to compare participants' activities on similar days, we limit our data to only those participants who completed a questionnaire on a weekday, during the term time, and on an ordinary/school day, although we also consider the full sample as a robustness check. Appendix Figure A1 shows the construction of the analysis dataset. 
We consider two measures of SES in our analysis: mother's education (highest level of education attained by the mother) and family income. Maternal education has been strongly linked to parental investment behaviours and children's development in the economics literature (Haveman and Wolfe, 1995; Guryan et al., 2008; Gimenez-Nadal and Molina, 2013; Prickett and Augustine, 2016). In the Irish context, maternal education is commonly used as a measure of parental SES (e.g. Nolan and Layte 2014; Madden 2017). Finally, maternal education stays relatively constant across waves, providing a stable measure of underlying SES. Unlike income, education is not subject to random or temporary shocks, meaning that a gain in education is more likely to indicate a real growth in status. Mother's education is categorized into 3 groups: less than secondary (mothers who do not have the leaving certificate), secondary (mothers who have completed the leaving certificate), and more than secondary (mothers who have some third level qualification). The proportion in each category is shown in Table 1 .

Our second measure of SES is household equivalized income, which provides information on whether the financial circumstances of families change across waves. In our data, changing income is especially interesting to examine because the two waves of the GUI coincided with the recession, with the first wave occurring just before any effects of the recession were felt and the second wave occurring at the height of the recession's impact (McGovern and Rokicki, 2018). Table 1 shows evidence of the recession, with a decrease in average father's employment and household income from wave 1 to wave 2. For our measure, we use annual disposable household income, which is recorded as total gross household income, including social benefits and less statutory deductions of income tax and social insurance contributions, obtained via a series of questions to the primary caretaker. This income is then equivalized to take household size and structure into account. The equivalence scales assign a weight of 1 to the first adult in the household, 0.66 to each subsequent adult (aged 14 and above living in the household) and 0.33 to each child (aged less than 14). Household equivalized income is finally calculated as disposable household income divided by the sum of the weights. This calculated measure is provided by the GUI in the data. Prices at the time of interview in wave 1 were not much changed by wave 2, so unsurprisingly, results involving income are virtually identical whether we adjust for inflation or not. In what follows we present results using the unadjusted income data. Income quintiles are defined relative to the sample, which is representative of the Irish population of families with 9-year olds in $2007 / 8$. 
Table 1. Sample characteristics and comparison to population

\begin{tabular}{|c|c|c|c|c|}
\hline & \multicolumn{2}{|c|}{ Wave 1} & \multicolumn{2}{|c|}{ Wave 2} \\
\hline & $\begin{array}{c}\text { Full survey } \\
\text { sample }^{\mathrm{a}} \\
\mathrm{N}=8,568\end{array}$ & $\begin{array}{c}\text { Analysis } \\
\text { sample }^{b} \\
\mathrm{~N}=3,923\end{array}$ & $\begin{array}{c}\text { Full survey } \\
\text { sample }^{\mathrm{a}} \\
\mathrm{N}=7,525\end{array}$ & $\begin{array}{c}\text { Analysis } \\
\text { sample }^{\text {b }} \\
\mathrm{N}=3,257\end{array}$ \\
\hline \multicolumn{5}{|l|}{ Mother's education (\%) } \\
\hline Less than Secondary & 30 & 31 & 20 & 20 \\
\hline Secondary & 36 & 37 & 38 & 40 \\
\hline More than secondary & 32 & 32 & 40 & 40 \\
\hline Not in household & 1 & 0 & 2 & 0 \\
\hline \multicolumn{5}{|l|}{ Father's education $(\%)$} \\
\hline Less than Secondary & 26 & 25 & 17 & 16 \\
\hline Secondary & 22 & 24 & 23 & 24 \\
\hline More than secondary & 28 & 29 & 31 & 31 \\
\hline Not in household & 17 & 16 & 18 & 18 \\
\hline Missing/No response & 7 & 5 & 12 & 10 \\
\hline \multicolumn{5}{|l|}{ Mother employment (\%) } \\
\hline Employed & 53 & 54 & 57 & 57 \\
\hline Not employed & 46 & 46 & 41 & 43 \\
\hline Not in household & 1 & 0 & 2 & 0 \\
\hline \multicolumn{5}{|l|}{ Father employment (\%) } \\
\hline Employed & 73 & 75 & 66 & 67 \\
\hline Not employed & 10 & 9 & 16 & 15 \\
\hline Not in household & 17 & 16 & 18 & 18 \\
\hline Mother's age <=39 (\%) & 50 & 48 & 24 & 25 \\
\hline Mother married (\%) & 82 & 84 & 81 & 82 \\
\hline Urban $(\%)$ & 45 & 42 & 45 & 43 \\
\hline Household income (€) [mean (SD)] & $\begin{array}{c}18994 \\
(12437)\end{array}$ & $\begin{array}{c}19182 \\
(12362)\end{array}$ & $\begin{array}{l}15974 \\
(9098)\end{array}$ & $\begin{array}{l}16179 \\
(8772)\end{array}$ \\
\hline Household size [mean (SD)] & $\begin{array}{c}4.7 \\
(1.2)\end{array}$ & $\begin{array}{c}4.7 \\
(1.2)\end{array}$ & $\begin{array}{c}4.7 \\
(1.2)\end{array}$ & $\begin{array}{c}4.6 \\
(1.2)\end{array}$ \\
\hline
\end{tabular}

Notes: "Married" indicates married or living together. Household income is equivalized by household size. ${ }^{a}$ Weighted with wave-specific GUI sample weights. ${ }^{b}$ Weighted with wave-specific GUI time use sample weights. 


\subsection{Descriptive Statistics}

Before applying more formal analysis, we begin by describing the basic patterns underlying how children spend their time. Figure 1 shows the proportion of children that engage in activities from $2 \mathrm{pm}$ to $10 \mathrm{pm}$ at waves 1 and 2 . We present data for boys and girls combined as the gender-stratified patterns are similar.

At ages 9 and 13 the school day ends at about 2:30pm and 4pm, respectively, for the majority of students. The immediate after-school period is often spent doing homework. The increase in the proportion of all children engaging in personal care at $6 \mathrm{pm}$ is most likely due to the consumption of an evening meal. From about $6 \mathrm{pm}$ onwards, time is spent using media, doing sport, playing, with family, and doing personal care related activities. Compared to 9-year-olds, 13-year-olds go to sleep later in the evening and are less likely to engage in sport and reading. In contrast, 13-year-olds are more likely to engage in homework, media, and family activities.

Next we establish whether there are any differences by family background in the raw data on activity participation. Figure 2 shows the difference in the proportion of girls (top) and boys (bottom) engaging in after-school activities by maternal education at ages 9 and 13. The y-axis is the proportion of girls/boys engaging in an activity whose mothers had completed more than secondary education minus the proportion of girls/boys engaging in an activity whose mothers had completed less than secondary education. Therefore, a positive value indicates that an activity is relatively more common at the specified time for children with more highly educated mothers. While few differences exist for girls at age 9 , gaps are present by age 13; girls in households with low maternal education are much more likely participate in media and in play - unstructured activities such as "hanging out with friends" - and less likely to participate in reading during the after-school hours of between $4 \mathrm{pm}$ and $10 \mathrm{pm}$. For sports, the pattern reverses from age 9 to 13: more girls from low SES backgrounds participate in sports at age 9 for most of the after school period, while at age 13 the opposite is true. Boys exhibit a broadly similar pattern.

We examine differences in minutes spent by maternal education and household income quintile for all activities in Appendix Tables A3 and A4. 

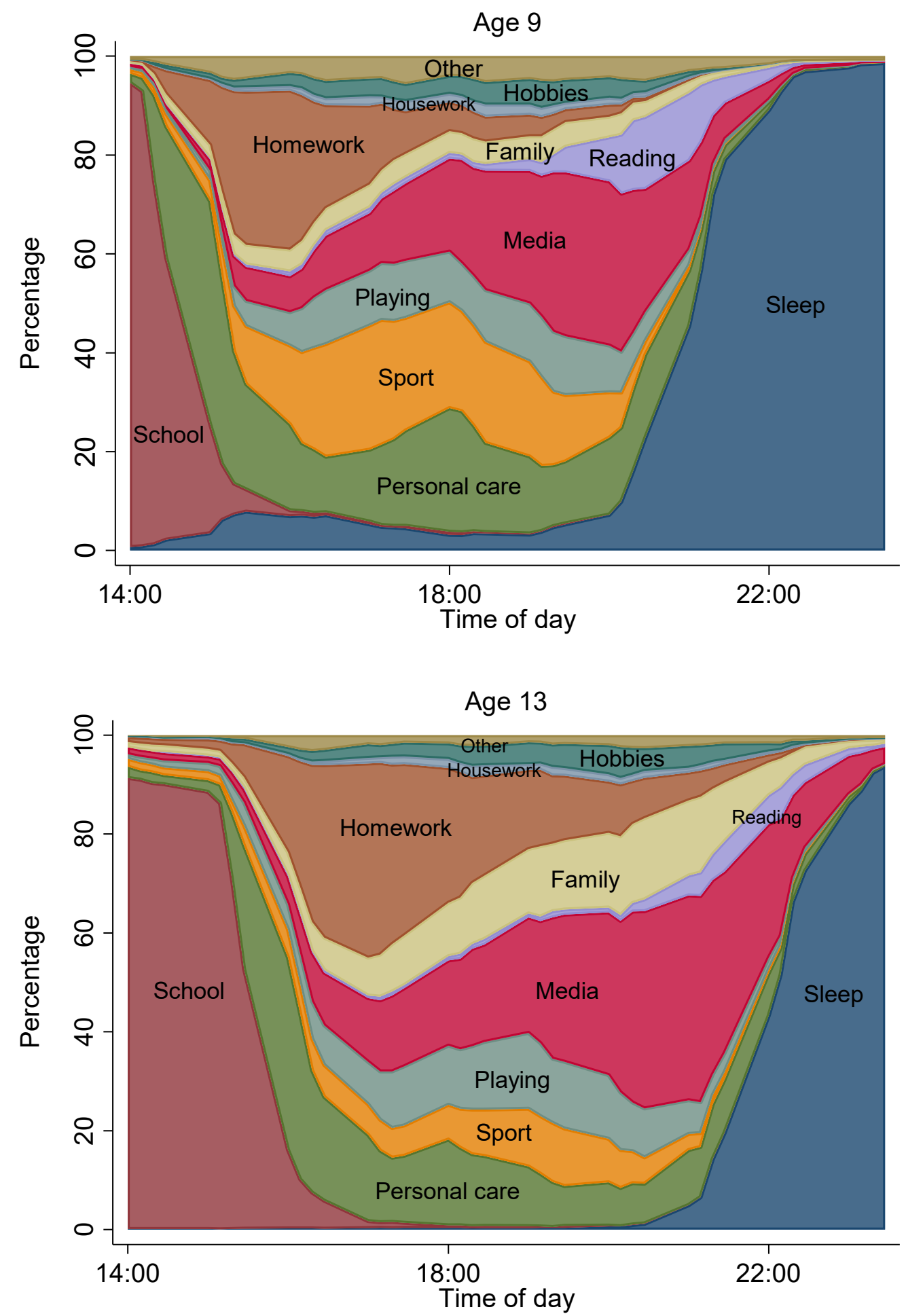

Fig. 1 Percentage of children participating in after-school activities at ages 9 (top) and 13 (bottom) 

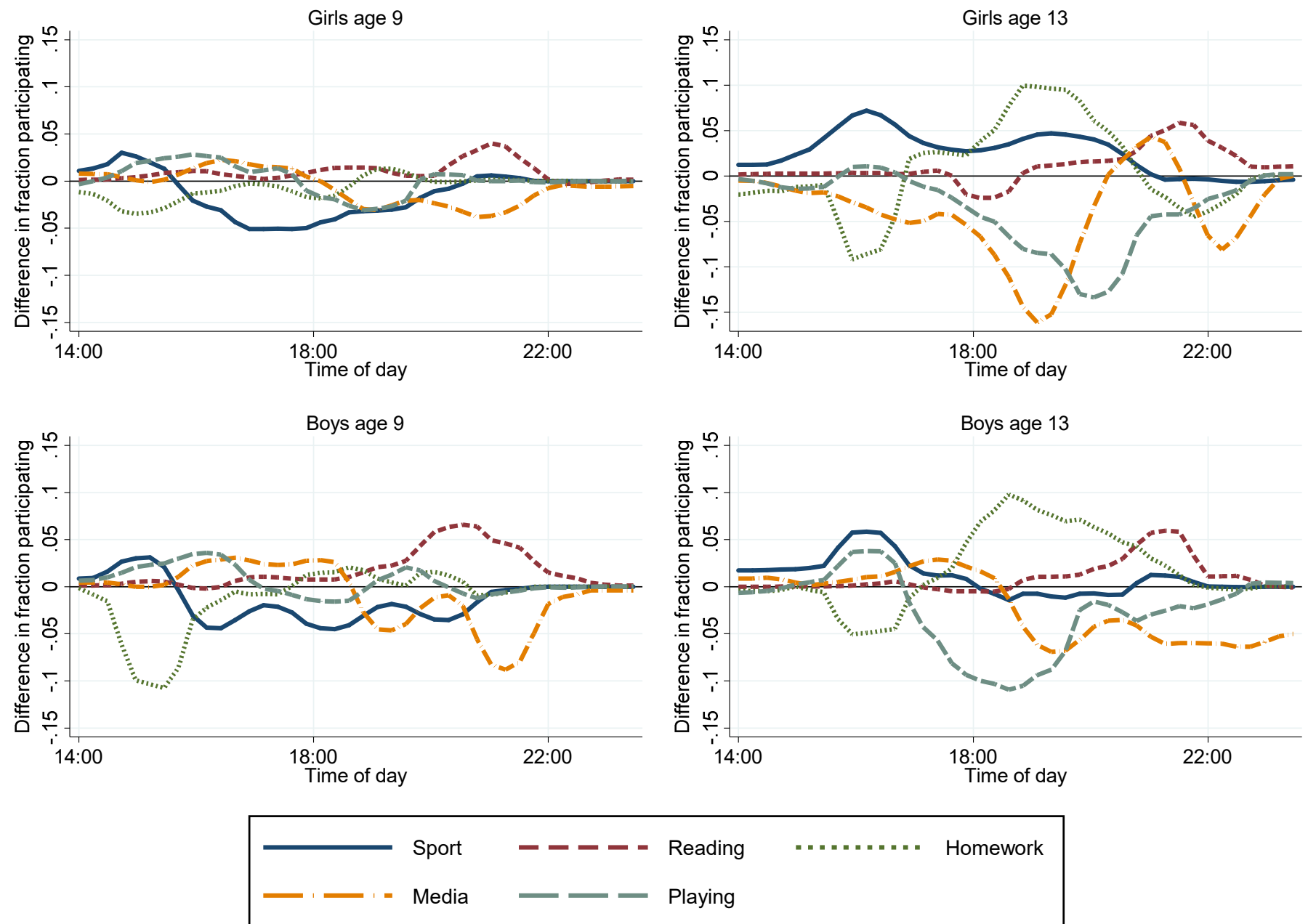

Fig. 2 Differences in children's participation at ages 9 and 13 in after-school activities for children in households with high maternal education compared to households with low maternal education

Notes: Difference calculated as fraction of children with mothers with more than secondary education participating in activity minus fraction of children with mothers with less than secondary education participating. Positive values indicate the activity is relatively more common for children with more highly educated mothers. 


\subsection{Describing SES Differences using Concentration Indices}

We use concentration indices to assess the degree of income-related inequality in the distribution of children's time use. The concentration index $(C)$ is calculated as twice the area between the concentration curve and the 45 degree line of equality, where the concentration curve describes the relationship between the cumulative share of equivalized household income and the cumulative share of time spent in a particular activity This is the standard approach in the literature on assessing the extent of inequality in a continuous outcome and has been used previously in a number of different contexts, including obesity (Walsh and Cullinan, 2015), vaccination (Doherty, Walsh and O'Neill, 2014), health in older populations (McGovern, 2014), economic insecurity (Rohde, Tang and Rao, 2014), and child height-for-age (Wagstaff, van Doorslaer and Watanabe, 2003).

Eq. 1 defines the concentration index as,

$$
C=1-2 \int_{0}^{1} L_{h}(p) d p
$$

where $L_{h}(p)$ is the concentration curve at $p$ percent of the population. The concentration index is bounded between $[-1,+1]$, with zero indicating perfect equality. A positive value indicates that lower income households receive less than their expected share of the minutes in each activity, while a negative value indicates they receive more than their expected share.

As described above, we focus on the three investment activities (reading, sport and homework) and compare these to the four leisure activities (media, playing, hobbies, and family). Appendix Figures A2-A8 show the concentration curves for each activity for girls and boys at ages 9 and 13. Table 2 presents the concentration indices for these, stratified by wave and gender. The largest concentration index is that for boys' sports time at 0.15 at age 13 . As an intuitive interpretation, concentration indices indicate the proportion of the outcome that would need to be redistributed from the richest half of households to the poorest half of households in order to achieve an equal distribution (concentration index of 0) (Koolman and Doorslaer, 2004). For example, if $15 \%$ of sports time was transferred from the richest half of households to the poorest half of households, the concentration index for boys would then be 0 . A concentration index of this magnitude for boys' sport is comparable to that for children's obesity in Ireland (Walsh and Cullinan, 2015), but is smaller than that for birth weight (Madden, 2014).

Overall, we find a high degree of income-related inequality for sports and reading at age 13 for both girls and boys. We find small to negligible inequality for all other activities across wave and gender, with the exception of hobbies for boys at age 9. These results differ from those in Figure 2, which found large time use differences for media and playing time by maternal education, indicating that maternal education may be a more important factor determining children's time use than income. 
Table 2. Concentration indices for girls and boys at ages 9 and 13

\begin{tabular}{cccccccc}
\hline & Sport & Reading & Homework & Media & Playing & Hobbies & Family \\
\hline $\begin{array}{c}\text { Panel A: Girls } \\
\text { Age 9 }\end{array}$ & 0.03 & 0.0004 & -0.03 & -0.01 & 0.002 & 0.03 & 0.07 \\
& $(0.02)$ & $(0.02)$ & $(0.01)$ & $(0.01)$ & $(0.02)$ & $(0.03)$ & $(0.04)$ \\
& & & & & & & \\
Age 13 & 0.11 & 0.07 & 0.01 & -0.002 & -0.06 & 0.06 & -0.01 \\
& $(0.03)$ & $(0.04)$ & $(0.01)$ & $(0.01)$ & $(0.02)$ & $(0.03)$ & $(0.02)$ \\
& & & & & & & \\
Panel B: Boys & & & & & & & \\
Age 9 & 0.01 & 0.04 & -0.01 & 0.01 & -0.04 & 0.13 & -0.10 \\
& $(0.01)$ & 0.02 & $(0.01)$ & $(0.01)$ & $(0.02)$ & $(0.03)$ & $(0.04)$ \\
& & & & & & & \\
Age 13 & 0.15 & 0.09 & 0.01 & -0.03 & -0.05 & -0.02 & -0.001 \\
& $(0.02)$ & $(0.04)$ & $(0.01)$ & $(0.01)$ & $(0.02)$ & $(0.04)$ & $(0.02)$ \\
\hline
\end{tabular}

Notes: Standard errors in parentheses.

\subsection{Visualizing changes in time use as children age}

As we have described in the introduction, a novel feature of the data is that we are able to track children's time use longitudinally as they age. For example, we can establish for every child whether they are participating in the same activity at each point in the day at age 13 as they were at age 9 . To describe these patterns, we construct a summary measure of substitutions, which we define as the average number of minutes that are transferred from an activity in wave 1 to another activity in the same time slot in wave 2 . These substitutions refer exclusively to how the same child changed their time use as they aged, as opposed to differences in average cohort time use (therefore information on children who were only present in a single wave is not used). To summarize this information, we sum over each child $(c)$, time slot $(t)$, and activity $(a)$, the number of minutes per day transferred from one activity to each of the other activities (Eq. 2).

$$
S u b_{a_{i j}}=\frac{15}{N} *\left(\sum_{c=1}^{N} \sum_{t=1}^{T} \sum_{i=1}^{12} \sum_{j=1}^{11} I\left(a_{c, t, i, \text { wave }=1}=a_{c, t, j, \text { wave }=2}\right)\right) \forall i \neq j
$$

Here, $a_{c, t, i, \text { wave }=1}$ is activity $i$ for child $c$ in timeslot $t$ in wave $1, a_{c, t, j, \text { wave }=2}$ is activity $j$ in the corresponding timeslot for the same child in wave 2 , and $I($.$) is the indicator function, evaluating$ whether these activities are the same. We multiply by 15 because the time slots are in 15 minute intervals. $N=1,585$, the total number of children present in both wave 1 and wave 2 , and $T=98$, the total number of 15-minute timeslots in a day. We report the net figures between activities, e.g. 10 minutes transferred from homework to reading and 5 minutes transferred from reading to homework results in an overall 5 minutes transferred from homework to reading. We aggregate some categories to simplify the presentation, so that all leisure activities (hobbies, media, family, playing) are categorized as "leisure". This measure is shown for all activities in matrix form as Table A5 in the Appendix and summarized in Figure 3 as a chord diagram. In this graph, the arrows indicate the direction of substitution from one activity to another, with the size of the arrows proportional to the average number of minutes transferred per day. The numbers around the outside give the total inflow and outflow of 
minutes for each activity. Note that most activities have both inflows and outflows. An exception is sleep, for which all of the total change (100 minutes per day) is an outflow. About 35 minutes of sleep are substituted with time in the 'other' category, while about the same amount of time is substituted away from 'other' to school, indicating that children are ultimately substituting sleep time with school. Another 35 minutes of sleep time are substituted with leisure time. In addition, we find that reading minutes flow mainly to leisure. For sport, the largest outflow is to homework, followed by leisure, school, and other.

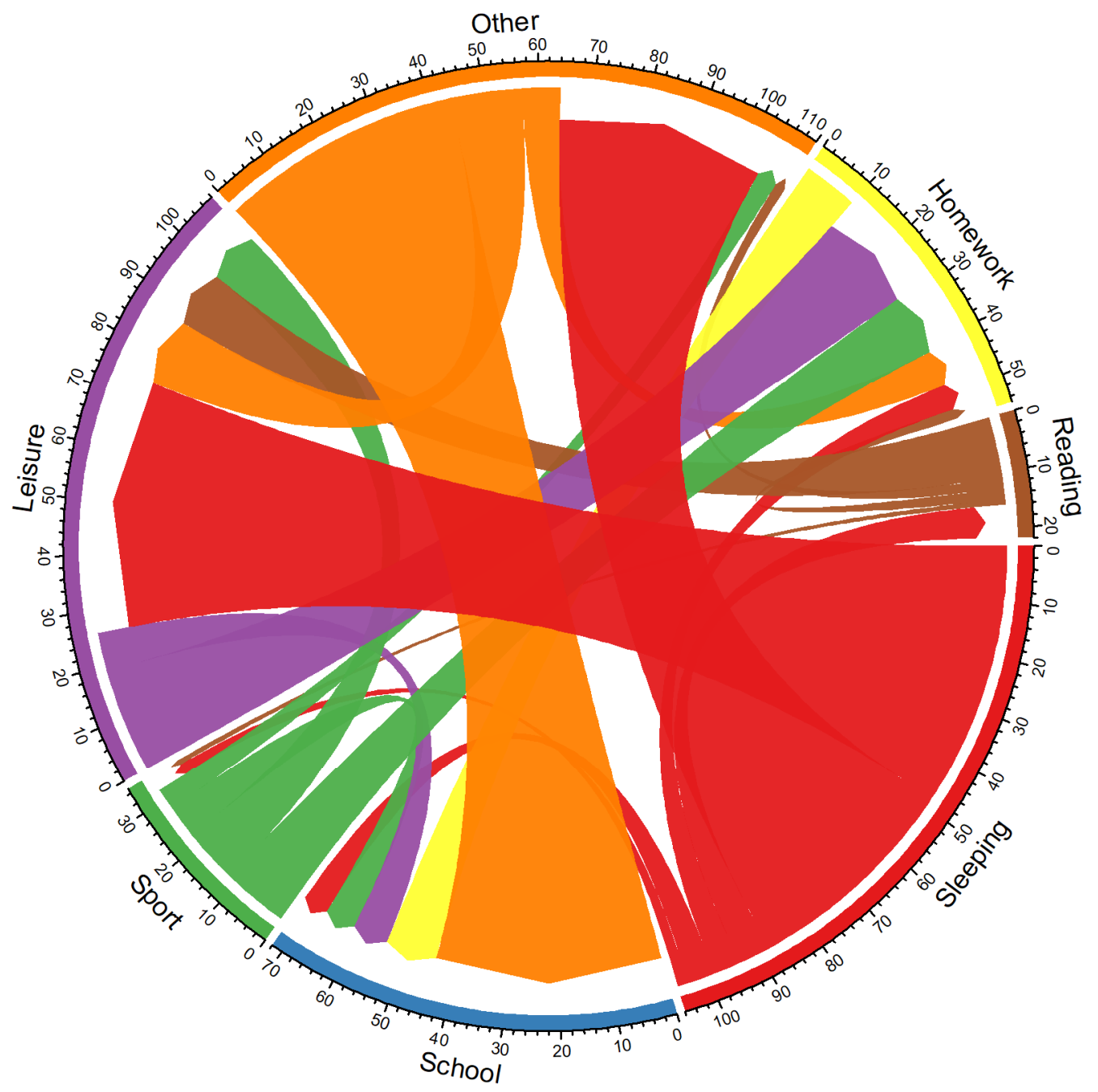

Fig. 3 Chord diagram of time use substitution patterns from age 9 to age 13.

Notes: Leisure includes playing (unstructured), media, family, and hobbies categories. Other includes personal care, housework, and "other" categories.

\subsection{Modelling Heterogeneity in Children's Time Use: Empirical strategy}

To test our two research hypotheses (whether SES differences are present in children's time use, and if so, whether they widen with age), we implement regression models which allow us to examine heterogeneity in children's time use at ages 9 and 13. The data allow us to consider both extensive and 
intensive margins of time use. We begin by examining heterogeneity in time use on the extensive margin, focusing on maternal education. We apply both pooled linear regression models and logistic regression models, with standard errors clustered at the child level and weighted with wave-specific GUI time use sample weights. In this setup, the outcome, $Y$, is a binary indicator for any time spent in a particular activity $a$ for child $c$ in period $t$. We include an interaction between mother's education for child $c$ in period $t$ and an indicator for wave, and also control for TUD characteristics (day of week, month of year, who completed diary, and when it was completed) in $Z_{c t}$. The specification for the linear model is shown below (Eq. 3):

$$
Y_{c t}^{a}=\alpha_{1}+\gamma_{1} \text { Wave }_{t}+\beta_{1} \text { MotherEd }_{c t}+\delta_{1} \text { Wave }_{t} * \text { MotherEd }_{c t}+Z_{c t} \mu_{1}^{\prime}+\varepsilon_{c t}
$$

In this model, the coefficients $\beta_{1}$ and $\delta_{1}$ allow us to test our hypotheses. If there are no SES differences in children's participation in activities at wave $1, \beta_{1}$ will be 0 . The coefficient $\delta_{1}$ estimates the additional change from wave 1 to wave 2. Therefore, if SES gaps in children's participation do not widen over time, $\delta_{1}$ will be 0 . We extend this model by examining alternative specifications, but in each case we will test the corresponding coefficients $\left(\beta_{2}\right.$ and $\delta_{2}$ in equation 4$)$ to assess whether the data support our two research hypotheses.

We next examine socioeconomic heterogeneity in time use on the intensive margin, again focusing on maternal education. We apply linear regression models with clustered standard errors at the child level. The outcome is the number of minutes spent in a particular activity and the right-hand side of the model is the same as above. The linear specification is shown in Eq. (4):

$$
\text { TimeUse }_{c t}^{a}=\alpha_{2}+\gamma_{2} \text { Wave }_{t}+\beta_{2} \text { MotherEd }_{c t}+\delta_{2} \text { Wave }_{t} * \text { MotherEd }_{c t}+Z_{c t} \mu_{2}^{\prime}+\varepsilon_{c t}
$$

where TimeUse $e_{c t}^{a}$ is the amount of minutes spent on a particular activity $a$ for child $c$ at time $t$. As a robustness check, we also apply generalized linear negative binomial models with a log-link with the same covariate specification. We use generalized linear modelling (GLM) instead of a two-part model because the negative binomial accounts for over-dispersion whilst allowing for more straightforward interpretation of coefficients without the requirement of splitting the sample. Previous research has found very little difference in performance between GLM and two-part models (Buntin and Zaslavsky, 2004). Moreover, we present separate results for participation. In both base-case extensive and intensive margin analyses, we do not control for covariates such as maternal employment, household income, or maternal marital status as these variables are likely to be on the causal pathway between maternal education and time use.

Next, we extend our model to explore possible mechanisms by which SES affects children's time use by including both mother's education and household income as measures of SES in the model, focusing on the intensive margin. The linear specification is shown in Eq. (5):

$$
\begin{aligned}
\text { TimeUse }_{c t}^{a}= & \alpha_{3}+\gamma_{3} \text { Wave }_{t}+\beta_{3} \text { MotherEd }_{c t}+\delta_{3} \text { Wave }_{t} * \text { MotherEd }_{c t} \\
& +\psi_{3} \text { LogIncome }_{c t}+\varphi_{3} \text { Wave }_{t} * \text { LogIncome }_{c t}+Z_{c t} \mu_{3}^{\prime}+X_{c t} v_{3}^{\prime}+\varepsilon_{c t}
\end{aligned}
$$

where LogIncome $c t$ is the log of equivalized household income for child $c$ at time $t$. In this model, we also additionally control for mother's and father's employment, mother's marital status, and household size in $X_{c t}$. We show results without these additional controls in the Appendix. We also show results of this model controlling for an even wider range of variables including mother's age, father's education, mother's and father's employment, mother's marital status, number of children in the household, and child health status in the Appendix. To assess the role of income as a mechanism, we examine whether the coefficients on maternal education $\left(\beta_{3}\right.$ and $\left.\delta_{3}\right)$ are attenuated with the addition of this additional covariate compared to the magnitude of the coefficients in equation 4 . We also test whether income plays an independent role in predicting differences in time use $\left(\psi_{3}\right)$, and again whether these differences grow as children age $\left(\varphi_{3}\right)$. 
Finally, we exploit the longitudinal nature of our data to investigate the impact of changes in SES on time use in the short-term by applying individual fixed effects (FE) models. The FE models control for unobserved time-invariant characteristics of children and families, such as initial conditions like birth weight and parental background. By eliminating potential omitted variable bias from unobserved factors that do not vary and that may confound the relationship between income and time use, we can better assess whether changes in income are likely to result in changes in time use. Because maternal education changes for less than $10 \%$ of families across waves, we focus instead on household income as our measure of SES. The fixed effects model is shown in Eq. 6:

$$
\text { TimeUse }_{c t}^{a}=\alpha_{c}+\delta_{t}+\beta \text { LnIncome }_{c t}+Z_{c t} \mu_{4}^{\prime}+X_{c t} v_{4}^{\prime}+\epsilon_{c t}
$$

where LnIncome $e_{c t}$ is the log household equivalized income for child $c$ at time t, $X_{c t}$ is the same matrix of time-varying variables as above, $\delta_{t}$ is the time fixed effect, and $\alpha_{c}$ is the child fixed effect. We show results with and without controlling for $X_{c t}$. We cluster standard errors at the child level.

Because we have a large number of outcomes, we adjust for multiple hypothesis testing. For all models, we adjust critical $\alpha$-levels using a family-wise Bonferroni method that adjusts for correlation in the outcomes, referred to as the Dubey/Armitage-Parmar (D/AP) procedure (Sankoh, Huque and Dubey, 1997). The "family" refers to all outcomes for a given gender in each model specification.

\section{Results}

\subsection{Extensive Margin}

Table 3 shows the results of the pooled linear regression models for girls and boys for participation in after-school activities as a function of maternal education. Logit and linear regression models reach similar conclusions (Appendix Tables A6 and A7 show the full set of coefficients for both models for girls and boys, respectively). Of note is that we find no difference in sports participation by mother's education for girls at age 9 , at which time about $46 \%$ of girls participate. While girls across all levels of SES decrease their sports participation from age 9 to 13, this trend is differential by mother's education: there is a 37 percentage point drop in participation among girls whose mothers have less than secondary education, while only a 22 percentage point drop for girls whose mothers have more than secondary education, a difference that appears substantial given the baseline participation rate.

Results for reading describe a different pattern. By age 9, a gap in reading participation by mother's education has already emerged: girls whose mothers have more than secondary education are 17 percentage points more likely to read for pleasure than girls whose mothers have less than secondary education. The corresponding odds ratio from the logit model indicates that girls whose mothers have more than secondary education are twice as likely to read in wave 1 as girls whose mothers have less than secondary education (Appendix Table A6). This reading gap persists at wave 2, but does not widen (the coefficient on the interaction between maternal education and wave is not statistically significant).

After adjusting for multiple hypothesis testing, there is no evidence of statistically significant differences in playing, media, family time, or homework participation across levels of maternal education in either wave. Girls whose mothers have more than secondary education are 14 percentage points more likely to participate in hobbies at age 9, though this gap narrows at age 13 .

Overall, the participation results for boys are similar to girls across activities, except for boys' sports participation, where there is no evidence of a gap by maternal education in either wave.

\subsection{Intensive Margin}

Table 4 shows the results of the linear regression models for girls and boys separately for the time spent in each activity as a function of maternal education. Coefficients from negative binomial models, which 
produce the same conclusions, and the full set of coefficients for linear regression models are shown in Appendix Tables A8 and A9 for girls and boys, respectively.

The gradients in time spent in activities by maternal education are similar to those in participation. In wave 1, girls whose mothers have more than secondary education spend 8 fewer minutes in sport. However, by wave 2 the gradient has reversed and girls whose mothers have more than secondary education spend $25-8=17$ additional minutes on sport per day. In the negative binomial model, compared with girls with mothers with less than secondary education, girls with mothers with completed secondary education spend twice as much time in sports while girls with mothers with more than secondary education spend roughly 3 times as much time daily doing sports at wave 2 (Appendix Table A8). As with participation, the maternal education reading gap is present at age 9 (7 minutes greater for girls with mothers with more than secondary education compared to less than secondary education). While no gap exists at age 9 for playing time, it is large at age 13 (20 fewer minutes for girls with mothers with more than secondary education compared to less than secondary education, a nearly $40 \%$ reduction). In fact, girls with mothers with secondary education and more than secondary education reduce their playing time from wave 1 to wave 2, while girls with mothers with less than secondary education increase their daily playing time over the waves.

For boys, results are similar, though there are a few noticeable differences. Though the coefficients on sports time and playing for mother's education show similar trends as for girls, the standard errors are larger. Additionally, we see substantial gaps in homework time which was not present for girls, with an additional $16-7=9$ minutes in wave 2 for boys whose mothers have more than secondary education compared to less than secondary education, an increase of $33 \%$.

Next, to examine possible mechanisms, we show the results of models that include both mother's education and household income as measures of SES (Table 5). These models also include further adjustment for mother's and father's employment, mother's marital status, and family size. Results for models without these additional controls were nearly identical and are shown in Appendix Table A10. Results with an even wider set of controls are shown in Appendix Table A11 and produce the same conclusions. We find that even after controlling for household income, gaps in time use by mother's education widen for sports time for girls and for homework and playing time for boys. We also find evidence of large reading gaps present at age 9 for both girls and boys by maternal education. With the addition of income into the model, we find that for boys, higher income is associated with more sports time at ages 9 and 13. However, income is not associated with time spent in any other activities for either girls or boys. 
Table 3. Linear regression results for participation in activities for girls and boys

\begin{tabular}{|c|c|c|c|c|c|c|c|c|c|c|c|c|c|c|}
\hline \multirow[b]{2}{*}{ Variables } & \multicolumn{7}{|c|}{ Girls } & \multicolumn{7}{|c|}{ Boys } \\
\hline & $\begin{array}{l}\text { Any } \\
\text { Sport }\end{array}$ & $\begin{array}{c}\text { Any } \\
\text { Reading }\end{array}$ & $\begin{array}{c}\text { Any } \\
\text { Homework }\end{array}$ & $\begin{array}{c}\text { Any } \\
\text { Playing }\end{array}$ & $\begin{array}{l}\text { Any } \\
\text { Media }\end{array}$ & $\begin{array}{c}\text { Any } \\
\text { Hobbies }\end{array}$ & $\begin{array}{c}\text { Any } \\
\text { Family }\end{array}$ & $\begin{array}{l}\text { Any } \\
\text { Sport }\end{array}$ & $\begin{array}{c}\text { Any } \\
\text { Reading }\end{array}$ & $\begin{array}{c}\text { Any } \\
\text { Homework }\end{array}$ & $\begin{array}{c}\text { Any } \\
\text { Playing }\end{array}$ & $\begin{array}{l}\text { Any } \\
\text { Media }\end{array}$ & $\begin{array}{c}\text { Any } \\
\text { Hobbies }\end{array}$ & $\begin{array}{l}\text { Any } \\
\text { Family }\end{array}$ \\
\hline Wave $=2$ & $\begin{array}{c}-0.37^{* * *} \\
(0.05)\end{array}$ & $\begin{array}{c}-0.26^{* * *} \\
(0.05)\end{array}$ & $\begin{array}{c}0.07 \\
(0.04)\end{array}$ & $\begin{array}{c}0.01 \\
(0.06)\end{array}$ & $\begin{array}{c}0.03 \\
(0.05)\end{array}$ & $\begin{array}{l}-0.01 \\
(0.05)\end{array}$ & $\begin{array}{c}0.34^{* * *} \\
(0.06)\end{array}$ & $\begin{array}{c}-0.32^{* * *} \\
(0.06)\end{array}$ & $\begin{array}{c}-0.20 * * * \\
(0.04)\end{array}$ & $\begin{array}{c}0.01 \\
(0.05)\end{array}$ & $\begin{array}{c}0.07 \\
(0.06)\end{array}$ & $\begin{array}{c}0.06 \\
(0.04)\end{array}$ & $\begin{array}{l}-0.02 \\
(0.03)\end{array}$ & $\begin{array}{c}0.38^{* * *} \\
(0.06)\end{array}$ \\
\hline $\begin{array}{l}\text { Mother's } \\
\text { Education }\end{array}$ & & & & & & & & & & & & & & \\
\hline Secondary & $\begin{array}{l}-0.01 \\
(0.04)\end{array}$ & $\begin{array}{l}0.11^{* *} \\
(0.04)\end{array}$ & $\begin{array}{l}-0.00 \\
(0.03)\end{array}$ & $\begin{array}{c}0.07 \\
(0.04)\end{array}$ & $\begin{array}{c}0.04 \\
(0.03)\end{array}$ & $\begin{array}{c}0.05 \\
(0.04)\end{array}$ & $\begin{array}{l}0.06^{*} \\
(0.03)\end{array}$ & $\begin{array}{c}0.03 \\
(0.04)\end{array}$ & $\begin{array}{l}0.11^{* *} \\
(0.04)\end{array}$ & $\begin{array}{l}-0.03 \\
(0.03)\end{array}$ & $\begin{array}{c}0.04 \\
(0.04)\end{array}$ & $\begin{array}{c}0.02 \\
(0.03)\end{array}$ & $\begin{array}{c}0.05 \\
(0.03)\end{array}$ & $\begin{array}{c}0.00 \\
(0.04)\end{array}$ \\
\hline $\begin{array}{l}\text { More than } \\
\text { Secondary }\end{array}$ & -0.03 & $0.17 * * *$ & -0.01 & 0.04 & 0.01 & $0.14 * * *$ & 0.04 & 0.05 & $0.25 * * *$ & $-0.07 * *$ & $0.09 * *$ & 0.02 & $0.12 * * *$ & -0.01 \\
\hline & $(0.04)$ & $(0.04)$ & $(0.02)$ & $(0.04)$ & $(0.03)$ & $(0.04)$ & $(0.03)$ & $(0.03)$ & $(0.04)$ & $(0.03)$ & $(0.04)$ & $(0.03)$ & $(0.03)$ & $(0.04)$ \\
\hline Secondary & & & & & & & & & & & & & & \\
\hline Wave 2 & 0.04 & -0.04 & -0.03 & -0.15 & -0.03 & -0.06 & 0.02 & 0.00 & -0.08 & 0.04 & -0.06 & -0.08 & 0.04 & -0.06 \\
\hline & $(0.06)$ & $(0.06)$ & $(0.05)$ & $(0.07)$ & $(0.05)$ & $(0.06)$ & $(0.07)$ & $(0.06)$ & $(0.05)$ & $(0.05)$ & $(0.07)$ & (0.05) & $(0.04)$ & $(0.06)$ \\
\hline $\begin{array}{l}\text { More than } \\
\text { Secondary }\end{array}$ & & & & & & & & & & & & & & \\
\hline Wave 2 & $0.15^{*}$ & -0.01 & -0.03 & -0.13 & -0.03 & -0.06 & -0.02 & -0.00 & -0.12 & 0.06 & -0.13 & -0.07 & 0.00 & -0.07 \\
\hline & $(0.06)$ & $(0.06)$ & $(0.04)$ & $(0.07)$ & $(0.05)$ & $(0.06)$ & $(0.06)$ & $(0.06)$ & $(0.05)$ & $(0.05)$ & $(0.07)$ & $(0.04)$ & $(0.04)$ & $(0.06)$ \\
\hline Constant & $\begin{array}{c}0.46^{* * *} \\
(0.06)\end{array}$ & $\begin{array}{c}0.47^{* * *} \\
(0.06)\end{array}$ & $\begin{array}{c}0.88^{* * *} \\
(0.04)\end{array}$ & $\begin{array}{c}0.51^{* * *} \\
(0.06)\end{array}$ & $\begin{array}{c}0.87^{* * *} \\
(0.04)\end{array}$ & $\begin{array}{c}0.27^{* * *} \\
(0.05)\end{array}$ & $\begin{array}{c}0.16^{* * *} \\
(0.05)\end{array}$ & $\begin{array}{c}0.61^{* * *} \\
(0.05)\end{array}$ & $\begin{array}{c}0.34^{* * *} \\
(0.05)\end{array}$ & $\begin{array}{c}0.92^{* * *} \\
(0.04)\end{array}$ & $\begin{array}{c}0.50^{* * *} \\
(0.06)\end{array}$ & $\begin{array}{c}0.85^{* * *} \\
(0.04)\end{array}$ & $\begin{array}{c}0.16^{* * *} \\
(0.04)\end{array}$ & $\begin{array}{c}0.21^{* * *} \\
(0.05)\end{array}$ \\
\hline $\mathrm{N}$ & 3,606 & 3,606 & 3,606 & 3,606 & 3,606 & 3,606 & 3,606 & 3,574 & 3,574 & 3,574 & 3,574 & 3,574 & 3,574 & 3,574 \\
\hline R-squared & 0.13 & 0.10 & 0.18 & 0.02 & 0.01 & 0.03 & 0.15 & 0.15 & 0.12 & 0.19 & 0.02 & 0.01 & 0.03 & 0.14 \\
\hline
\end{tabular}

Notes: Omitted category for mother's education is less than secondary. Clustered errors in parentheses. P-value alpha values of 0.01 , 0.05 , and 0.1 adjusted for multiple

hypothesis testing using D/AP procedure with correlation 0.05 resulting in $\mathrm{p}$-value cut-offs: $* * * \mathrm{p}<0.0016, * * \mathrm{p}<0.008, * \mathrm{p}<0.016$ 
Table 4. Linear regression results for time spent in activities for girls and boys

\begin{tabular}{|c|c|c|c|c|c|c|c|c|c|c|c|c|c|c|}
\hline \multirow[b]{2}{*}{ Variables } & \multicolumn{7}{|c|}{ Girls } & \multicolumn{7}{|c|}{ Boys } \\
\hline & $\begin{array}{l}\text { Sport } \\
\text { (Min) }\end{array}$ & $\begin{array}{l}\text { Reading } \\
\text { (Min) }\end{array}$ & $\begin{array}{l}\text { Homework } \\
\text { (Min) }\end{array}$ & $\begin{array}{l}\text { Playing } \\
\text { (Min) }\end{array}$ & $\begin{array}{l}\text { Media } \\
\text { (Min) }\end{array}$ & $\begin{array}{l}\text { Hobbies } \\
\text { (Min) }\end{array}$ & $\begin{array}{l}\text { Family } \\
\text { (Min) }\end{array}$ & $\begin{array}{l}\text { Sport } \\
\text { (Min) }\end{array}$ & $\begin{array}{c}\text { Reading } \\
\text { (Min) }\end{array}$ & $\begin{array}{l}\text { Homework } \\
\text { (Min) }\end{array}$ & $\begin{array}{l}\text { Playing } \\
\text { (Min) }\end{array}$ & $\begin{array}{c}\text { Media } \\
\text { (Min) }\end{array}$ & $\begin{array}{l}\text { Hobbies } \\
\text { (Min) }\end{array}$ & $\begin{array}{l}\text { Family } \\
\text { (Min) }\end{array}$ \\
\hline Wave $=2$ & $\begin{array}{c}-40.80 * * * \\
(4.13)\end{array}$ & $\begin{array}{c}-9.02 * * \\
(2.93)\end{array}$ & $\begin{array}{c}31.88^{* * *} \\
(6.81)\end{array}$ & $\begin{array}{l}14.15 \\
(7.35)\end{array}$ & $\begin{array}{c}41.68^{* * *} \\
(11.00)\end{array}$ & $\begin{array}{c}6.91 \\
(5.35)\end{array}$ & $\begin{array}{c}28.56^{* * *} \\
(6.19)\end{array}$ & $\begin{array}{c}-38.76 * * * \\
(6.44)\end{array}$ & $\begin{array}{c}-7.65^{* * *} \\
(2.16)\end{array}$ & $\begin{array}{c}15.52 * * \\
(5.50)\end{array}$ & $\begin{array}{l}19.27 \\
(8.38)\end{array}$ & $\begin{array}{c}38.16^{* * *} \\
(8.52)\end{array}$ & $\begin{array}{l}-1.12 \\
(2.60)\end{array}$ & $\begin{array}{c}37.41 * * * \\
(8.62)\end{array}$ \\
\hline $\begin{array}{l}\text { Mother's } \\
\text { Education }\end{array}$ & & & & & & & & & & & & & & \\
\hline Secondary & $\begin{array}{l}-5.28 \\
(4.38)\end{array}$ & $\begin{array}{c}3.97 \\
(2.17)\end{array}$ & $\begin{array}{l}-0.16 \\
(2.54)\end{array}$ & $\begin{array}{c}0.95 \\
(4.36)\end{array}$ & $\begin{array}{c}2.77 \\
(4.21)\end{array}$ & $\begin{array}{c}3.52 \\
(2.67)\end{array}$ & $\begin{array}{c}3.66 \\
(2.87)\end{array}$ & $\begin{array}{l}-1.67 \\
(5.54)\end{array}$ & $\begin{array}{c}4.09 \\
(1.87)\end{array}$ & $\begin{array}{l}-4.09 \\
(2.66)\end{array}$ & $\begin{array}{c}2.34 \\
(3.86)\end{array}$ & $\begin{array}{l}-2.21 \\
(4.95)\end{array}$ & $\begin{array}{c}0.77 \\
(2.05)\end{array}$ & $\begin{array}{l}-1.92 \\
(4.96)\end{array}$ \\
\hline $\begin{array}{l}\text { More than } \\
\text { Secondary }\end{array}$ & $\begin{array}{l}-8.37 \\
(4.25)\end{array}$ & $\begin{array}{c}6.82 * * * \\
(1.95)\end{array}$ & $\begin{array}{l}-3.39 \\
(2.36)\end{array}$ & $\begin{array}{l}-0.25 \\
(4.13)\end{array}$ & $\begin{array}{l}-2.08 \\
(4.01)\end{array}$ & $\begin{array}{c}7.87^{* * *} \\
(2.44)\end{array}$ & $\begin{array}{c}1.61 \\
(2.96)\end{array}$ & $\begin{array}{l}-3.42 \\
(5.10)\end{array}$ & $\begin{array}{c}11.40 * * * \\
(2.04)\end{array}$ & $\begin{array}{c}-6.94 * * \\
(2.45)\end{array}$ & $\begin{array}{c}3.34 \\
(3.52)\end{array}$ & $\begin{array}{l}-4.22 \\
(4.84)\end{array}$ & $\begin{array}{c}4.47 \\
(2.00)\end{array}$ & $\begin{array}{l}-4.04 \\
(4.77)\end{array}$ \\
\hline $\begin{array}{l}\text { Secondary * } \\
\text { Wave } 2\end{array}$ & $\begin{array}{c}14.15^{* *} \\
(5.37)\end{array}$ & $\begin{array}{l}-2.17 \\
(3.23)\end{array}$ & $\begin{array}{c}2.36 \\
(7.25)\end{array}$ & $\begin{array}{l}-16.98 \\
(8.31)\end{array}$ & $\begin{array}{l}-12.90 \\
(11.98)\end{array}$ & $\begin{array}{l}-9.06 * \\
(5.48)\end{array}$ & $\begin{array}{c}4.73 \\
(7.04)\end{array}$ & $\begin{array}{c}20.00 \\
(11.44)\end{array}$ & $\begin{array}{l}-2.81 \\
(2.67)\end{array}$ & $\begin{array}{l}13.35 \\
(6.27)\end{array}$ & $\begin{array}{l}-8.21 \\
(9.51)\end{array}$ & $\begin{array}{r}-10.53 \\
(9.99)\end{array}$ & $\begin{array}{c}5.89 \\
(3.49)\end{array}$ & $\begin{array}{l}-8.96 \\
(9.54)\end{array}$ \\
\hline $\begin{array}{l}\text { More than } \\
\text { Secondary * } \\
\text { Wave } 2\end{array}$ & $\begin{array}{c}25.38 * * * \\
(5.15)\end{array}$ & $\begin{array}{l}0.09 \\
(3.36)\end{array}$ & $\begin{array}{c}3.36 \\
(7.19)\end{array}$ & $\begin{array}{r}-20.26 * \\
(8.03)\end{array}$ & $\begin{array}{l}-20.13 \\
(11.56)\end{array}$ & $\begin{array}{l}-9.09 \\
(5.29)\end{array}$ & $\begin{array}{l}-0.73 \\
(6.84)\end{array}$ & $\begin{array}{l}11.71 \\
(7.04)\end{array}$ & $\begin{array}{l}-5.19 \\
(2.79)\end{array}$ & $\begin{array}{c}16.10 * * \\
(5.96)\end{array}$ & $\begin{array}{r}-18.50 \\
(8.78)\end{array}$ & $\begin{array}{l}-11.92 \\
(9.56)\end{array}$ & $\begin{array}{c}2.37 \\
(3.00)\end{array}$ & $\begin{array}{l}-9.45 \\
(9.14)\end{array}$ \\
\hline Constant & $\begin{array}{c}35.11^{* * *} \\
(5.51)\end{array}$ & $\begin{array}{c}22.25^{* * *} \\
(3.90)\end{array}$ & $\begin{array}{c}52.17^{* * *} \\
(4.45)\end{array}$ & $\begin{array}{c}40.92^{* * *} \\
(7.47)\end{array}$ & $\begin{array}{c}84.91^{* * *} \\
(8.50)\end{array}$ & $\begin{array}{c}17.94^{* * *} \\
(4.68)\end{array}$ & $\begin{array}{l}11.08 \\
(5.23)\end{array}$ & $\begin{array}{c}46.15^{* * *} \\
(7.82)\end{array}$ & $\begin{array}{c}15.31 * * * \\
(2.63)\end{array}$ & $\begin{array}{c}52.83^{* * *} \\
(4.41)\end{array}$ & $\begin{array}{c}31.36 * * * \\
(5.56)\end{array}$ & $\begin{array}{c}84.99 * * * \\
(7.47)\end{array}$ & $\begin{array}{c}9.79 * * * \\
(2.85)\end{array}$ & $\begin{array}{l}15.67 \\
(6.74)\end{array}$ \\
\hline$N$ & 3,606 & 3,606 & 3,606 & 3,606 & 3,606 & 3,606 & 3,606 & 3,574 & 3,574 & 3,574 & 3,574 & 3,574 & 3,574 & 3,574 \\
\hline R-squared & 0.14 & 0.05 & 0.17 & 0.04 & 0.07 & 0.02 & 0.11 & 0.12 & 0.07 & 0.15 & 0.03 & 0.06 & 0.02 & 0.11 \\
\hline
\end{tabular}

Notes: Omitted category for mother's education is less than secondary. Clustered errors in parentheses. P-value alpha values of $0.01,0.05$, and 0.1 adjusted for multiple hypothesis testing using D/AP procedure according with correlation=0.15 resulting in $\mathrm{p}$-value cut-offs: $* * \mathrm{p}<0.0019, * * \mathrm{p}<0.0096, * \mathrm{p}<0.02$ 
Table 5. Linear regression results for time spent in activities for girls and boys including both maternal education and income

\begin{tabular}{|c|c|c|c|c|c|c|c|c|c|c|c|c|c|c|}
\hline \multirow[b]{2}{*}{ Variables } & \multicolumn{7}{|c|}{ Girls } & \multicolumn{7}{|c|}{ Boys } \\
\hline & $\begin{array}{l}\text { Sport } \\
\text { (Min) }\end{array}$ & $\begin{array}{l}\text { Reading } \\
\text { (Min) }\end{array}$ & $\begin{array}{l}\text { Homework } \\
\text { (Min) }\end{array}$ & $\begin{array}{c}\text { Playing } \\
\text { (Min) }\end{array}$ & $\begin{array}{l}\text { Media } \\
\text { (Min) }\end{array}$ & $\begin{array}{l}\text { Hobbies } \\
\text { (Min) }\end{array}$ & $\begin{array}{l}\text { Family } \\
\text { (Min) }\end{array}$ & $\begin{array}{l}\text { Sport } \\
\text { (Min) }\end{array}$ & $\begin{array}{l}\text { Reading } \\
\text { (Min) }\end{array}$ & $\begin{array}{l}\text { Homework } \\
\text { (Min) }\end{array}$ & $\begin{array}{c}\text { Playing } \\
\text { (Min) }\end{array}$ & $\begin{array}{l}\text { Media } \\
\text { (Min) }\end{array}$ & $\begin{array}{l}\text { Hobbies } \\
\text { (Min) }\end{array}$ & $\begin{array}{l}\text { Family } \\
\text { (Min) }\end{array}$ \\
\hline Wave $=2$ & $\begin{array}{c}-36.88 * * * \\
(4.67)\end{array}$ & $\begin{array}{c}-8.47^{* *} \\
(3.00)\end{array}$ & $\begin{array}{c}34.85 * * * \\
(7.69)\end{array}$ & $\begin{array}{l}11.90 \\
(8.43)\end{array}$ & $\begin{array}{c}36.51 * * * \\
(10.74)\end{array}$ & $\begin{array}{c}5.84 \\
(5.79)\end{array}$ & $\begin{array}{c}28.98^{* * *} \\
(6.65)\end{array}$ & $\begin{array}{c}-30.84 * * * \\
(7.12)\end{array}$ & $\begin{array}{l}-5.65^{*} \\
(2.34)\end{array}$ & $\begin{array}{l}11.13 \\
(6.12)\end{array}$ & $\begin{array}{c}22.27 * \\
(8.98)\end{array}$ & $\begin{array}{c}36.12^{* * *} \\
(9.49)\end{array}$ & $\begin{array}{l}-4.62 \\
(2.65)\end{array}$ & $\begin{array}{c}37.25^{* * *} \\
(8.14)\end{array}$ \\
\hline Mother's Education & & & & & & & & & & & & & & \\
\hline Secondary & $\begin{array}{l}-2.83 \\
(4.36)\end{array}$ & $\begin{array}{c}5.02 \\
(2.41)\end{array}$ & $\begin{array}{l}-0.77 \\
(2.67)\end{array}$ & $\begin{array}{c}0.00 \\
(4.52)\end{array}$ & $\begin{array}{c}4.63 \\
(4.45)\end{array}$ & $\begin{array}{c}2.72 \\
(2.72)\end{array}$ & $\begin{array}{c}3.50 \\
(3.14)\end{array}$ & $\begin{array}{l}-5.51 \\
(5.88)\end{array}$ & $\begin{array}{l}4.91^{*} \\
(1.90)\end{array}$ & $\begin{array}{l}-4.10 \\
(2.81)\end{array}$ & $\begin{array}{c}4.55 \\
(3.89)\end{array}$ & $\begin{array}{l}-3.56 \\
(5.26)\end{array}$ & $\begin{array}{l}-0.28 \\
(2.12)\end{array}$ & $\begin{array}{c}0.03 \\
(5.08)\end{array}$ \\
\hline More than secondary & $\begin{array}{l}-7.48 \\
(4.56)\end{array}$ & $\begin{array}{c}7.45 * * * \\
(2.25)\end{array}$ & $\begin{array}{l}-2.20 \\
(2.59)\end{array}$ & $\begin{array}{l}-0.57 \\
(4.38)\end{array}$ & $\begin{array}{l}-0.53 \\
(4.48)\end{array}$ & $\begin{array}{c}5.96 \\
(2.66)\end{array}$ & $\begin{array}{c}1.04 \\
(3.02)\end{array}$ & $\begin{array}{l}-9.19 \\
(5.71)\end{array}$ & $\begin{array}{c}11.84^{* * *} \\
(2.20)\end{array}$ & $\begin{array}{c}-7.04 * * \\
(2.70)\end{array}$ & $\begin{array}{c}6.63 \\
(3.60)\end{array}$ & $\begin{array}{l}-5.85 \\
(5.39)\end{array}$ & $\begin{array}{c}2.71 \\
(2.11)\end{array}$ & $\begin{array}{l}-1.89 \\
(4.98)\end{array}$ \\
\hline Secondary * Wave 2 & $\begin{array}{c}9.73 \\
(5.62)\end{array}$ & $\begin{array}{l}-2.65 \\
(3.27)\end{array}$ & $\begin{array}{c}1.41 \\
(7.82)\end{array}$ & $\begin{array}{r}-15.20 \\
(8.81)\end{array}$ & $\begin{array}{c}-9.76 \\
(11.31)\end{array}$ & $\begin{array}{l}-7.48 \\
(5.72)\end{array}$ & $\begin{array}{c}3.59 \\
(7.31)\end{array}$ & $\begin{array}{l}10.42 \\
(8.04)\end{array}$ & $\begin{array}{l}-3.76 \\
(2.85)\end{array}$ & $\begin{array}{c}17.32^{* *} \\
(6.58)\end{array}$ & $\begin{array}{l}-12.32 \\
(9.93)\end{array}$ & $\begin{array}{c}-9.63 \\
(10.71)\end{array}$ & $\begin{array}{c}9.52^{* *} \\
(3.62)\end{array}$ & $\begin{array}{l}-10.69 \\
(8.95)\end{array}$ \\
\hline $\begin{array}{l}\text { More than Secondary } \\
\text { * Wave } 2\end{array}$ & $\begin{array}{c}21.94 * * * \\
(5.78)\end{array}$ & $\begin{array}{l}-0.08 \\
(3.40)\end{array}$ & $\begin{array}{l}-0.08 \\
(8.02)\end{array}$ & $\begin{array}{r}-17.87 \\
(9.07)\end{array}$ & $\begin{array}{l}-14.70 \\
(11.51)\end{array}$ & $\begin{array}{l}-8.34 \\
(6.01)\end{array}$ & $\begin{array}{l}-2.32 \\
(7.11)\end{array}$ & $\begin{array}{c}7.07 \\
(7.63)\end{array}$ & $\begin{array}{l}-6.41 \\
(2.95)\end{array}$ & $\begin{array}{c}18.41^{* *} \\
(6.66)\end{array}$ & $\begin{array}{c}-25.08 * * \\
(9.39)\end{array}$ & $\begin{array}{c}-9.40 \\
(10.70)\end{array}$ & $\begin{array}{c}6.62 \\
(3.10)\end{array}$ & $\begin{array}{l}-9.58 \\
(8.83)\end{array}$ \\
\hline Log income & $\begin{array}{c}0.67 \\
(6.25)\end{array}$ & $\begin{array}{l}-0.00 \\
(1.88)\end{array}$ & $\begin{array}{l}-2.30 \\
(2.11)\end{array}$ & $\begin{array}{c}0.52 \\
(3.47)\end{array}$ & $\begin{array}{l}-0.65 \\
(3.75)\end{array}$ & $\begin{array}{c}0.08 \\
(2.18)\end{array}$ & $\begin{array}{l}-2.04 \\
(2.73)\end{array}$ & $\begin{array}{c}10.41 * \\
(4.02)\end{array}$ & $\begin{array}{l}-0.79 \\
(1.64)\end{array}$ & $\begin{array}{l}-0.27 \\
(2.39)\end{array}$ & $\begin{array}{l}-4.10 \\
(3.35)\end{array}$ & $\begin{array}{c}1.51 \\
(4.38)\end{array}$ & $\begin{array}{c}1.57 \\
(1.51)\end{array}$ & $\begin{array}{l}-5.14 \\
(3.28)\end{array}$ \\
\hline Log income * Wave 2 & $\begin{array}{c}0.25 \\
(6.49)\end{array}$ & $\begin{array}{c}0.63 \\
(3.01)\end{array}$ & $\begin{array}{c}4.30 \\
(5.02)\end{array}$ & $\begin{array}{c}-2.27 \\
(6.26)\end{array}$ & $\begin{array}{c}4.36 \\
(7.62)\end{array}$ & $\begin{array}{c}2.94 \\
(3.23)\end{array}$ & $\begin{array}{l}-1.75 \\
(5.15)\end{array}$ & $\begin{array}{l}16.19 \\
(7.26)\end{array}$ & $\begin{array}{c}3.01 \\
(2.37)\end{array}$ & $\begin{array}{l}-1.79 \\
(4.73)\end{array}$ & $\begin{array}{c}2.51 \\
(5.90)\end{array}$ & $\begin{array}{r}-14.35 \\
(7.53)\end{array}$ & $\begin{array}{l}-6.12 \\
(2.81)\end{array}$ & $\begin{array}{c}6.31 \\
(5.75)\end{array}$ \\
\hline Observations & 3,338 & 3,338 & 3,338 & 3,338 & 3,338 & 3,338 & 3,338 & 3,340 & 3,340 & 3,340 & 3,340 & 3,340 & 3,340 & 3,340 \\
\hline R-squared & 0.14 & 0.06 & 0.18 & 0.04 & 0.07 & 0.02 & 0.12 & 0.16 & 0.07 & 0.16 & 0.06 & 0.07 & 0.03 & 0.11 \\
\hline
\end{tabular}

Notes: Regressions adjusted for mother's and father's employment, mother's marital status, family size, and time diary characteristics. Omitted category for mother's

education is less than secondary. Clustered errors in parentheses. P-value alpha values of 0.01, 0.05, and 0.1 adjusted for multiple hypothesis testing using D/AP procedure according with correlation $=0.15$ resulting in $\mathrm{p}$-value cut-offs: $* * * \mathrm{p}<0.0019, * * \mathrm{p}<0.0096, * \mathrm{p}<0.02$ 
Table 6 shows the results of the fixed effects regressions of time use on income for girls (panel A) and boys (panel B). We show models adjusted only for time diary characteristics and models that include additional controls. The full set of coefficients for these models are shown in Appendix Tables A12 and A13.

After adjusting for multiple hypothesis testing, none of the coefficients on income for either girls or boys is significantly different from 0 for any activity in the fixed effects models, indicating that a change in income from age 9 to 13 is not associated with a change in children's time use. We return to the interpretation of these results in the discussion. 
Table 6. Results of fixed effects models evaluating changes in household income and time spent in activities for girls and boys

\begin{tabular}{|c|c|c|c|c|c|c|c|c|c|c|c|c|c|c|}
\hline & $\begin{array}{c}\text { Sports } \\
\text { (min) }\end{array}$ & $\begin{array}{c}\text { Sports } \\
\text { (min) }\end{array}$ & $\begin{array}{l}\text { Reading } \\
\text { (min) }\end{array}$ & $\begin{array}{c}\text { Reading } \\
\text { (min) }\end{array}$ & $\begin{array}{l}\text { Homework } \\
\quad(\min )\end{array}$ & $\begin{array}{l}\text { Homework } \\
\text { (min) }\end{array}$ & $\begin{array}{c}\text { Playing } \\
\text { (min) }\end{array}$ & $\begin{array}{c}\text { Playing } \\
\text { (min) }\end{array}$ & $\begin{array}{c}\text { Media } \\
(\min )\end{array}$ & $\begin{array}{c}\text { Media } \\
(\mathrm{min})\end{array}$ & $\begin{array}{c}\text { Hobbies } \\
\text { (min) }\end{array}$ & $\begin{array}{c}\text { Hobbies } \\
\quad(\min )\end{array}$ & $\begin{array}{c}\text { Family } \\
\text { (min) }\end{array}$ & $\begin{array}{c}\text { Family } \\
\text { (min) }\end{array}$ \\
\hline \multicolumn{15}{|l|}{ Panel A: Girls } \\
\hline Log income & $\begin{array}{l}-1.98 \\
(6.21)\end{array}$ & $\begin{array}{l}-5.40 \\
(6.33)\end{array}$ & $\begin{array}{c}1.83 \\
(3.14)\end{array}$ & $\begin{array}{c}2.89 \\
(3.48)\end{array}$ & $\begin{array}{c}0.20 \\
(3.94)\end{array}$ & $\begin{array}{c}1.89 \\
(4.07)\end{array}$ & $\begin{array}{c}5.38 \\
(5.80)\end{array}$ & $\begin{array}{c}5.21 \\
(6.21)\end{array}$ & $\begin{array}{c}0.06 \\
(7.11)\end{array}$ & $\begin{array}{c}0.19 \\
(7.51)\end{array}$ & $\begin{array}{l}-6.16 \\
(4.24)\end{array}$ & $\begin{array}{l}-7.67 \\
(4.52)\end{array}$ & $\begin{array}{l}-4.27 \\
(5.29)\end{array}$ & $\begin{array}{l}-4.04 \\
(5.49)\end{array}$ \\
\hline Adjusted & $\mathrm{N}$ & $\mathrm{Y}$ & $\mathrm{N}$ & $\mathrm{Y}$ & $\mathrm{N}$ & $\mathrm{Y}$ & $\mathrm{N}$ & $\mathrm{Y}$ & $\mathrm{N}$ & Y & $\mathrm{N}$ & $\mathrm{Y}$ & $\mathrm{N}$ & $\mathrm{Y}$ \\
\hline Number of ID & 1,764 & 1,764 & 1,764 & 1,764 & 1,764 & 1,764 & 1,764 & 1,764 & 1,764 & 1,764 & 1,764 & 1,764 & 1,764 & 1,764 \\
\hline \multicolumn{15}{|l|}{ Panel B: Boys } \\
\hline Log income & $\begin{array}{l}-3.88 \\
(6.85)\end{array}$ & $\begin{array}{l}-2.09 \\
(7.34)\end{array}$ & $\begin{array}{l}-3.53 \\
(2.62)\end{array}$ & $\begin{array}{l}-3.91 \\
(2.56)\end{array}$ & $\begin{array}{l}-1.34 \\
(4.89)\end{array}$ & $\begin{array}{l}-2.36 \\
(4.91)\end{array}$ & $\begin{array}{l}-1.86 \\
(6.20)\end{array}$ & $\begin{array}{l}-2.07 \\
(6.31)\end{array}$ & $\begin{array}{l}-3.17 \\
(7.74)\end{array}$ & $\begin{array}{l}-2.57 \\
(8.23)\end{array}$ & $\begin{array}{l}-1.98 \\
(4.25)\end{array}$ & $\begin{array}{l}-0.78 \\
(4.28)\end{array}$ & $\begin{array}{c}5.52 \\
(6.18)\end{array}$ & $\begin{array}{c}1.45 \\
(6.56)\end{array}$ \\
\hline Adjusted & $\mathrm{N}$ & $\mathrm{Y}$ & $\mathrm{N}$ & $\mathrm{Y}$ & $\mathrm{N}$ & $\mathrm{Y}$ & $\mathrm{N}$ & $\mathrm{Y}$ & $\mathrm{N}$ & $\mathrm{Y}$ & $\mathrm{N}$ & $\mathrm{Y}$ & $\mathrm{N}$ & $\mathrm{Y}$ \\
\hline Number of ID & 1,770 & 1,770 & 1,770 & 1,770 & 1,770 & 1,770 & 1,770 & 1,770 & 1,770 & 1,770 & 1,770 & 1,770 & 1,770 & 1,770 \\
\hline
\end{tabular}

Notes: Income is household income, equivalised for family size. Clustered errors in parentheses. Unadjusted regression models adjusted only for time diary characteristics. Adjusted models additionally adjusted for mother's and father's employment, mother's marital status, and family size. P-value alpha values of $0.01,0.05$, and 0.1 adjusted for multiple hypothesis testing using D/AP procedure with correlation=0.15 resulting in $\mathrm{p}$-value cut-offs: $* * * \mathrm{p}<0.0019, * * \mathrm{p}<0.0096, * \mathrm{p}<0.02$ 


\section{Discussion}

We conducted a longitudinal analysis of heterogeneity in children's time use to understand differences in activity participation by family background. In particular, we examined two main research hypotheses. First, we determined the extent to which socioeconomic gaps in children's time use exist. Second, we examined how these gaps change over time. We focus on comparing activities that can be most viewed as investments in human capital which have been shown in the literature to be beneficial for skill acquisition (reading, sports and homework time) with leisure activities.

Our main results highlight several key messages. First, in terms of our first research hypothesis, we identify substantial differences in sports, reading, homework, and playing time by socioeconomic status that are largely consistent with the theoretical framework outlined in Section 2. At age 9, girls from high SES backgrounds spend 7 more minutes each day reading than girls from low SES backgrounds. At age 13, girls with high SES backgrounds spend 17 more minutes each day on sports and 20 fewer minutes each day on unstructured playing time than girls from low SES backgrounds. A similar pattern exists for boys, where we also find differences in homework time. Both linear and corresponding nonlinear models for participation and time spent in activities show consistent results (Tables 2 and 3).

Second, in terms of our second research hypothesis, we find that while gaps in children's time use at age 9 are generally small, they widen as children age for sports, homework, and playing time (Tables 2 and 3). For example, girls from low SES backgrounds spend slightly more time in sports at age 9, but by age 13 the trend has reversed. Similarly, there is no difference in playing time at age 9 , but while girls with mothers with low education increase their daily playing time from age 9 to 13 , girls with mothers with average and with high education decrease this time, resulting in a large gap. We find a similar systematic widening of gaps for boys.

Our results for reading, however, do not support our second hypothesis. Gaps in reading time are already formed by age 9 and stay stable from age 9 to 13. Reading requires skills formed in early childhood (Sparks, Patton and Murdoch, 2014), and so it is not surprising that gaps in reading time are already present at age 9. However, it may be surprising that these gaps do not widen over time, as expected for an investment activity. One explanation may be due to the aggregate large drop in reading time, which is essentially halved from wave 1 to wave 2 for both girls and boys across all levels of SES. Figure 2 and Appendix Table A5 show that most of reading time at age 9 is shifted to media time at age 13, which increased 20-40 minutes per day across levels of SES. The period of 2007-2012 saw significant changes in technology, with rapid increases in broadband internet connections and smartphone ownership as well as the proliferation of social media and smartphone applications (Pew Research Center, n.d.). These large technological changes, occurring concurrently with children's growth from middle childhood into young adolescence, may have resulted in a substantial cohort shift in time use from reading to technology and media. In our data, the percentage of children who have a mobile phone increased from $45 \%$ at age 9 to $59 \%$ at age 13. Findings from the EU Kids Online survey conducted in 2010 found that while only $28 \%$ of 9-10 year olds have a social media profile, 59\% of 11-12 year olds do, suggesting that the start of secondary school may increase correspond to an increase in the use of social networking (Livingstone et al., 2010).

Third, we find that for boys, household income is significantly associated with sports' time (Table 4). For this activity, economically disadvantaged parents may be limited by the cost of equipment and fees for formal sports. The GUI data confirm that sports time is increasingly made up of formal organized activity as children grow older (as opposed to, for example, exercising through playing outside with friends at age 9). Removing or subsidising equipment or membership costs could therefore reduce income-related inequality in sports participation for boys. In the US, these costs are estimated at $10 \%$ of annual income for two-child families in the bottom income quintile (Putnam, 2016). Previous research in Ireland is consistent with this hypothesis, as a report on obesity and physical activity among the 13 -year-old cohort found that $37 \%$ of children in the lowest social group never participated in 
organized sports, compared to $17 \%$ in the highest social group (ESRI, 2012). Collecting further data on these costs could provide a basis for determining whether there is justification for policy intervention in this area.

Finally, aside from boys' sports we find no association of income with time spent in any other activities for boys or girls, while we find strong associations between maternal education and children's time use. This points to parental education being a much more important factor in family investment decisions about children's time use than income, and is in line with previous evidence on differences in parenting styles and values by parental education (Lareau, 2003; Sayer et al., 2004; Guryan et al., 2008; GimenezNadal and Molina, 2013). For example, gaps in parental time spent with children by parental education have been shown to be quite similar across countries despite substantial cross-national variation in levels of economic support and services for families (Sayer et al., 2004).

An important consideration is that while we find that income is associated with boys' sports time in the pooled linear model (Table 4), these results do not hold in the fixed effects model (Table 5). The fixed effects model captures changes in income from wave 1 to wave 2, while the pooled linear regression model captures levels of income at waves 1 and 2. During the time period under study in our data (20072012), the Great Recession resulted in a substantial income shock for many households and affected poorer households more severely than better-off households. Yet despite these shocks, we do not see an impact on children's time use. This may be because these income shocks were of a temporary nature rather than representing a fall in permanent income. If permanent income matters more than transitory income, the implication is that we would be unlikely to see short run income gains resulting in a closing of the SES gradient in time use.

Examining the patterns of socioeconomic differences in time use may be useful for forming policy recommendations. Though no socioeconomic gap exists in sports and exercise participation at age 9, large differences emerge by age 13, particularly for girls. Conversely, for girls' and boys' reading time, the gap already exists by age 9 . Therefore, for policies that seek to reduce socioeconomic inequality in girls' sports time, it may be most effective to aim to intervene before the time of puberty (normally at ages 12-14), when the fall-off in sports participation is greatest, while for policies that aim to reduce inequality in reading skills, it may be most effective to intervene before age 9 . This would be consistent with the theoretical framework we outline in section 3, which suggests there can be a high degree of path dependency in skill acquisition. Given the literature on the importance of childhood development for adult outcomes, there may be important implications of SES time use differences for social mobility.

Moreover, the SES inequalities in sports time, particularly for girls, are likely to have important implications for health. Previous research has found strong gradients in BMI and obesity by socioeconomic status within the GUI child cohort at both ages 9 and 13, with the gradient becoming steeper for girls at age 13 (Madden, 2017). Moreover, among 13-year-olds that participated in no sports or exercise activities, common reasons cited for non-participation were, "I am no good at games" and "I feel people laugh at me because of my size" (ESRI, 2012).

Our analysis has a number of limitations. First, time diary data, though more accurate than stylized questions that estimate a respondent's time spent in activities, may suffer from measurement error, for example due to reporting bias. Second, the results may be affected by attrition and selection into who completes the TUD. The families in our analyses are a subset of those in the full sample and not all of those who completed the TUD data in wave 1 also completed the TUD in wave 2. Descriptive statistics do suggest some differences in the characteristics of those in the TUD data compared to the main survey; however, weights are provided to make the TUD families representative of the population as a whole. When we compared results in our analyses with and without weights we found conclusions were similar regardless of whether we adjusted for this selection. However, these weights can only account for observed characteristics; if families are selecting into the TUD data or dropping out of the survey in wave 2 on the basis of unobserved factors which are also correlated with the relationships of interest 
(here, SES and time use), this could affect our estimates. Selection on unobserved variables is difficult to assess without an appropriate selection variable (which affects participation but not the outcome), which is not available in our data. Therefore, we cannot test this directly in our data and it is important to bear this limitation in mind. However, we do control for diary characteristics and additional robustness checks including those participants who completed diaries on weekend days give very similar results (see Appendix Table A14). Future validation exercises for the survey testing attrition, in particular by family strata, would be beneficial. Third, we implement fixed effects models as a way of accounting for all time-invariant omitted variables such as family history and child initial conditions. However, there are also drawbacks to this approach in that we are not able to control for time-varying confounders, it is less efficient, and measurement error is a much greater concern in these models than random effects or pooled OLS, potentially leading to substantial attenuation bias (Kohler, Behrman and Schnittker, 2011). Additionally, pooled models include observations from participants present in any wave, while FE models necessitate participants being present in both waves. Therefore, it is important to be cautious when interpreting results from these models and investigate causality in future research using alternative identification assumptions, for instance by using natural experiments which affect the activities available to children and their families (Castro, 2019).

An additional research and policy question that we did not pursue here is how school-level variation may affect educational opportunity and therefore time use. Schools may differ, for example, in the amount, difficulty, or expectations relating to assigned homework. The Irish school system could be considered relatively homogenous compared to other countries in that $92 \%$ of children attend churchrun primary schools (which are state supported and do not charge fees) and typically attend their local institution. Almost all secondary schools are state funded and nominally free to the public. Both primary and second level education in Ireland is overseen by a single body (the Department of Education and Skills); all institutions follow a common curriculum and participate in state-wide examinations. However, there is undoubtedly school-level variation in educational instruction, demonstrated, for example, by differences in school performance in the Junior and Leaving Certificate tests (the national state exams at the middle and end of secondary school). Although likely to be less of an issue at primary level, there is the potential for parental selection of schools at second level. Separating the influence of schools from the influence of family-level characteristics is an interesting question. However, because we are interested in documenting how gradients in time use by family background change over time in this paper, we do not wish to adjust for school characteristics as part of this analysis. Parental decisions taken about where to send children for secondary school are likely to partly represent outcomes of socioeconomic background characteristics and therefore adjusting for secondary school characteristics could potentially understate social gradients in the outcomes we examine. We therefore leave questions about the school-level influences on time use for future analysis.

Finally, more research on the consequences of these inequalities is needed to understand the impact of differences in time use on inequalities in future educational attainment and social and labour outcomes. For example, unstructured playing time may be beneficial in that it may increase independence, resilience, and social competence; however, little empirical work has examined this relationship. Future research should also further examine families' preferences and beliefs regarding which types of investments are optimal, the extent to which different types of investments are substitutes or complements (Leibowitz, 2003), how investments reinforce or reduce the effects of initial endowments (Almond and Mazumder, 2013), and the causal relationship between participation in certain activities and subsequent outcomes across the life course. Estimating the technology of skill formation is an active area of investigation (Cunha and Heckman, 2008). Such research would inform the extent to which policy interventions should target the time use differences we document and whether such policies could be used to improve children's life chances. 
References

Almond, D., and B. Mazumder, "Fetal Origins and Parental Responses," Annual Review of Economics, 5, 37-56, 2013.

Altintas, E., "Widening education-gap in developmental childcare activities in the US," Journal of Marriage and Family, 78, 26-42, 2016.

Barron, J.M., B.T. Ewing, and G.R. Waddell, "The Effects of High School Athletic Participation on Education and Labor Market Outcomes," Review of Economics and Statistics, 82, 409-421, 2000 .

Becker, G.S., and N. Tomes, "Human Capital and the Rise and Fall of Families," Journal of Labor Economics, 4, 1-47, 1986.

Bianchi, S.M., J.P. Robinson, and M.A. Milke, The changing rhythms of American family life, Russell Sage Foundation, 2006.

Black, S.E., P.J. Devereux, and K.G. Salvanes, "From the Cradle to the Labor Market? The Effect of Birth Weight on Adult Outcomes," The Quarterly Journal of Economics, 122, 409-439, 2007.

Boardman, J.D., D.A. Powers, Y.C. Padilla, and R.A. Hummer, "Low birth weight, social factors, and developmental outcomes among children in the United States," Demography, 39, 353-368, 2002.

Bradley, R.H., and R.F. Corwyn, "Socioeconomic status and child development," Annual review of psychology, 53, 371-399, 2002.

Buntin, M.B., and A.M. Zaslavsky, "Too much ado about two-part models and transformation?: Comparing methods of modeling Medicare expenditures," Journal of Health Economics, 23, 525-542, 2004.

Burdette, H.L., and R.C. Whitaker, "Resurrecting Free Play in Young Children: Looking Beyond Fitness and Fatness to Attention, Affiliation, and Affect," Archives of Pediatrics \& Adolescent Medicine, 159, 46-50, 2005.

Burton, P., and S. Phipps, "Families, Time and Money in Canada, Germany, Sweden, the United Kingdom and the United States," Review of Income and Wealth, 53, 460-483, 2007.

Castro, A.N., "Television, time use and academic achievement: Evidence from a natural experiment," Granger Centre Discussion Paper No. 19/06, 2019.

Cunha, F., and J.J. Heckman, "Formulating, Identifying and Estimating the Technology of Cognitive and Noncognitive Skill Formation," Journal of Human Resources, 43, 738-782, 2008.

Currie, J., "Inequality at Birth: Some Causes and Consequences," American Economic Review, 101, $1-22,2011$.

Delaney, L., and O. Doyle, "Socioeconomic differences in early childhood time preferences," Journal of Economic Psychology, 33, 237-247, 2012.

Delaney, L., M. McGovern, and J.P. Smith, "From Angela's ashes to the Celtic tiger: Early life conditions and adult health in Ireland," Journal of Health Economics, 30, 1-10, 2011. 
DeMattia, L., L. Lemont, and L. Meurer, "Do interventions to limit sedentary behaviours change behaviour and reduce childhood obesity? A critical review of the literature," Obesity Reviews, 8, 69-81, 2007.

DiPrete, T.A., and G.M. Eirich, "Cumulative advantage as a mechanism for inequality: A review of theoretical and empirical developments," Annual Review of Sociology, 32, 271-297, 2006.

Doherty, E., B. Walsh, and C. O'Neill, "Decomposing socioeconomic inequality in child vaccination: Results from Ireland," Vaccine, 32, 3438-3444, 2014.

Doyle, O., L. McEntee, and K.A. McNamara, "Skills, capabilities and inequalities at school entry in a disadvantaged community," European journal of psychology of education, 27, 133-154, 2012.

ESRI, “Growing Up In Ireland. Key Findings: 13-Year-Olds. No. 2 Physical Activity and Obesity Among 13-Year-Olds.," Economic and Social Research Institute, Available at: http://www.esri.ie/pubs/OPEA113.pdf [Accessed November 21, 2017], 2012.

Fiorini, M., "The effect of home computer use on children's cognitive and non-cognitive skills," Economics of Education Review, 29, 55-72, 2010.

Fiorini, M., and M.P. Keane, "How the Allocation of Children's Time Affects Cognitive and Noncognitive Development," Journal of Labor Economics, 32, 787-836, 2014.

Fox, L., W. Han, C. Ruhm, and J. Waldfogel, "Time for Children: Trends in the Employment Patterns of Parents, 1967-2009,” Demography, 50, 25-49, 2013.

Fredricks, J.A., and J.S. Eccles, "Is extracurricular participation associated with beneficial outcomes? Concurrent and longitudinal relations," Developmental Psychology, 42, 698-713, 2006.

Gimenez-Nadal, J.I., and J.A. Molina, "Parents' education as a determinant of educational childcare time," Journal of Population Economics, 26, 719-749, 2013.

Gortmaker, S.L., A. Must, J.M. Perrin, A.M. Sobol, and W.H. Dietz, "Social and economic consequences of overweight in adolescence and young adulthood," New England journal of medicine, 329, 1008-1012, 1993.

Guryan, J., E. Hurst, and M. Kearney, "Parental education and parental time with children," The Journal of Economic Perspectives, 22, 23-46, 2008.

Gustafsson, B., and U. Kjulin, "Time use in child care and housework and the total cost of children," Journal of Population Economics, 7, 287-306, 1994.

Hallberg, D., and A. Klevmarken, “Time for children: A study of parent's time allocation," Journal of Population Economics, 16, 205-226, 2003.

Hamermesh, D.S., "Timing, togetherness and time windfalls," Journal of population Economics, 15, 601-623, 2002.

Harding, J.F., P.A. Morris, and D. Hughes, "The relationship between maternal education and children's academic outcomes: A theoretical framework," Journal of Marriage and Family, 77, 60-76, 2015.

Haveman, R., and B. Wolfe, "The Determinants of Children's Attainments: A Review of Methods and Findings," Journal of Economic Literature, 33, 1829-1878, 1995. 
Heckman, J.J., “Schools, skills, and synapses,” Economic inquiry, 46, 289-324, 2008.

Heckman, J.J., and D.V. Masterov, "The productivity argument for investing in young children," Applied Economic Perspectives and Policy, 29, 446-493, 2007.

Hofferth, S.L., and J.F. Sandberg, "How American Children Spend Their Time," Journal of Marriage and Family, 63, 295-308, 2001.

Hsin, A., "Is Biology Destiny? Birth Weight and Differential Parental Treatment," Demography, 49, 1385-1405, 2012.

Hsin, A., and C. Felfe, "When Does Time Matter? Maternal Employment, Children's Time With Parents, and Child Development," Demography, 51, 1867-1894, 2014.

Kalenkoski, C.M., D.C. Ribar, and L.S. Stratton, "The influence of wages on parents' allocations of time to child care and market work in the United Kingdom," Journal of Population Economics, 22, 399-419, 2009.

Kalil, A., R. Ryan, and M. Corey, "Diverging destinies: Maternal education and the developmental gradient in time with children," Demography, 49, 1361-1383, 2012.

Kan, M.Y., and S. Pudney, "Measurement Error in Stylized and Diary Data on Time Use," Sociological Methodology, 38, 101-132, 2008.

Kling, J.R., J.B. Liebman, and L.F. Katz, "Experimental Analysis of Neighborhood Effects," Econometrica, 75, 83-119, 2007.

Kohler, H.-P., J.R. Behrman, and J. Schnittker, "Social science methods for twins data: Integrating causality, endowments, and heritability," Biodemography and Social Biology, 57, 88-141, 2011.

Koolman, X., and E. van Doorslaer, "On the Interpretation of a Concentration Index of Inequality," Health Economics, 13, 649-56, 2004.

Lareau, A., "Unequal childhoods: Race, class, and family life," Berkeley: University of California Press, 2003.

Leibowitz, A., "Home Investments in Children," Journal of Political Economy, 82, S111-S131, 1974.

Leibowitz, A.A., "In-home training and the production of children's human capital," Review of Economics of the Household, 1, 305-317, 2003.

Lipscomb, S., "Secondary school extracurricular involvement and academic achievement: a fixed effects approach," Economics of Education Review, 26, 463-472, 2007.

Livingstone, S., L. Haddon, A. Gorzig, and K. Olafsson, "Risks and safety for children on the internet: the UK report. Full findings from the EU Kids Online survey of UK 9-16 year olds and their parents.," European Union and London School of Economics and Political Science, Available at:

http://eprints.lse.ac.uk/53058/1/_lse.ac.uk_storage_LIBRARY_Secondary_libfile_shared_re pository_Content_EU\%20Kids\%20Online_EU_Kids_Online_Report_Risks_and_safety_for_ children_on_the_internet_2010.pdf [Accessed June 13, 2019], 2010.

Madden, D., "Childhood obesity and maternal education in Ireland," Economics and Human Biology, 27, 114-125, 2017. 
Madden, D., "The relationship between low birth weight and socioeconomic status in Ireland," Journal of Biosocial Science, 46, 248-265, 2014.

McGovern, M., and S. Rokicki, "The Great Recession, Household Income, and Children's Test Scores," Geary Institute Working Paper WP2018/15, 2018.

McGovern, M.E., "Comparing the Relationship Between Stature and Later Life Health in Six Low and Middle Income Countries," The Journal of the Economics of Ageing, 4, 128-148, 2014.

McGovern, M.E., "Still Unequal at Birth: Birth Weight, Socioeconomic Status and Outcomes at Age 9," Economic and Social Review, 44, 53-84, 2013.

McLanahan, S., "Diverging destinies: How children are faring under the second demographic transition," Demography, 41, 607-627, 2004.

Nolan, A., and R. Layte, "Socio-economic Inequalities in Child Health in Ireland," Economic and Social Review, 45, 25-64, 2014.

Perkins, D.F., J.E. Jacobs, B.L. Barber, and J.S. Eccles, "Childhood and adolescent sports participation as predictors of participation in sports and physical fitness activities during young adulthood," Youth \& Society, 35, 495-520, 2004.

Pew Research Center, "Three Technology Revolutions," Internet and Technology, Available at: http://www.pewinternet.org/three-technology-revolutions/ [Accessed October 11, 2018],

Pfeifer, C., and T. Cornelißen, "The impact of participation in sports on educational attainment-New evidence from Germany," Economics of Education Review, 29, 94-103, 2010.

Posner, J.K., and D.L. Vandell, "After-school activities and the development of low-income urban children: A longitudinal study,” Developmental Psychology, 35, 868-879, 1999.

Prickett, K.C., and J.M. Augustine, "Maternal Education and Investments in Children's Health," Journal of Marriage and Family; Minneapolis, 78, 7-25, 2016.

Putnam, R.D., Our kids: The American dream in crisis, New York: Simon and Schuster, 2016.

Quail, A., and J. Williams, "Data Available from the Time-Use Survey, Wave 1 of the Child Cohort in Growing Up in Ireland," Economic and Social Research Institute, 2013.

Quail, A., and J. Williams, "Data Available from the Time-Use Survey, Wave 2 of the Child Cohort in Growing Up in Ireland," Economic and Social Research Institute, 2015.

Ramey, G., and V.A. Ramey, “The Rug Rat Race,” Brookings Papers on Economic Activity, 1, 129$176,2010$.

Rapoport, B., and C. Le Bourdais, "Parental time and working schedules," Journal of Population Economics, 21, 903-932, 2008.

Rohde, N., K.K. Tang, and D.S.P. Rao, "Distributional Characteristics of Income Insecurity in the U.S., Germany, and Britain," Review of Income and Wealth, 60, S159-S176, 2014.

Sankoh, A.J., M.F. Huque, and S.D. Dubey, "Some comments on frequently used multiple endpoint adjustment methods in clinical trials," Statistics in Medicine, 16, 2529-2542, 1997. 
Sayer, L.C., S.M. Bianchi, and J.P. Robinson, "Are Parents Investing Less in Children? Trends in Mothers' and Fathers' Time with Children1," American journal of sociology, 110, 1-43, 2004.

Smith, J.P., "The Impact of Childhood Health on Adult Labor Market Outcomes," Review of Economics and Statistics, 91, 478-489, 2009.

Sousa-Poza, A., H. Schmid, and R. Widmer, "The allocation and value of time assigned to housework and child-care: An analysis for Switzerland," Journal of Population Economics, 14, 599-618, 2001 .

Sparks, R.L., J. Patton, and A. Murdoch, "Early reading success and its relationship to reading achievement and reading volume: replication of '10 years later," Reading and Writing, 27, 189-211, 2014.

Stevenson, B., "Beyond the classroom: Using Title IX to measure the return to high school sports," The Review of Economics and Statistics, 92, 284-301, 2010.

Tandon, P.S., C. Zhou, J.F. Sallis, K.L. Cain, L.D. Frank, and B.E. Saelens, "Home environment relationships with children's physical activity, sedentary time, and screen time by socioeconomic status," International Journal of Behavioral Nutrition and Physical Activity, 9, 88, 2012.

Telama, R., X. Yang, J. Viikari, I. Välimäki, O. Wanne, and O. Raitakari, "Physical activity from childhood to adulthood: a 21-year tracking study," American journal of preventive medicine, 28, 267-273, 2005.

Wagstaff, A., E. van Doorslaer, and N. Watanabe, "On decomposing the causes of health sector inequalities with an application to malnutrition inequalities in Vietnam," Journal of Econometrics, 112, 207-223, 2003.

Walsh, B., and J. Cullinan, "Decomposing socioeconomic inequalities in childhood obesity: Evidence from Ireland," Economics \& Human Biology, 16, 60-72, 2015.

Wight, V.R., J. Price, S.M. Bianchi, and B.R. Hunt, "The time use of teenagers," Social Science Research, 38, 792-809, 2009. 


\section{Appendix}

Table A1 Categorization of wave 1 and wave 2 activities

\begin{tabular}{|c|c|c|}
\hline Category & Wave 1 & Wave 2 \\
\hline Sleeping & $\begin{array}{l}\text { *Sleeping } \\
* \text { Resting/relaxing }\end{array}$ & *Sleeping/Resting \\
\hline $\begin{array}{l}\text { Personal } \\
\text { Care }\end{array}$ & $\begin{array}{l}\text { *Personal care } \\
\text { *Eating/drinking/having a meal } \\
\text { *Traveling to and from school } \\
\text { *Other traveling }\end{array}$ & $\begin{array}{l}\text { *Personal care or getting ready } \\
\text { *Eating } \\
\text { *Traveling }\end{array}$ \\
\hline School & $*$ At school & $*$ At school \\
\hline Homework & *Homework & *Doing homework or study \\
\hline Sport & *Physical play/exercise/sports & $\begin{array}{l}* \text { Playing sport or doing physical } \\
\text { exercise }\end{array}$ \\
\hline $\begin{array}{l}\text { Playing/ } \\
\text { Unstructured } \\
\text { time }\end{array}$ & $\begin{array}{l}\text { *Playing board games, cards, etc } \\
\text { *General play }\end{array}$ & $\begin{array}{l}\text { *Just hanging around with friends } \\
\text { *Playing with or exercising a pet }\end{array}$ \\
\hline Hobbies & *Hobbies and other leisure activities & $\begin{array}{l}\text { *Music Lessons (or practicing } \\
\text { music), drama, classes } \\
\text { *Hobbies and other leisure activities }\end{array}$ \\
\hline Media & $\begin{array}{l}* \text { Computer/internet/playstation/xbox } \\
* \text { Email/bebo/msn/texting/on the } \\
\text { phone } \\
* \text { Watching tv and videos/dvds etc }\end{array}$ & $\begin{array}{l}\text { *Using the internet/emailing } \\
\text { *Playing computer games } \\
\text { *Talking on the phone or texting } \\
\text { *Watching tv, films, videos, or dvds } \\
\text { *Listening to music }\end{array}$ \\
\hline Reading & *Reading books, comics, magazines & $*$ Reading for pleasure or interest \\
\hline Housework & *Household chores/housework & *Housework \\
\hline Family & $\begin{array}{l}* \text { Visits to relative's house for } \\
\text { purposes other than play } \\
* \text { On a family outing } \\
* \text { On a shopping trip }\end{array}$ & $\begin{array}{l}\text { *Spending time with family } \\
* \text { On an outing } \\
* \text { Out shopping to buy things }\end{array}$ \\
\hline Other & $\begin{array}{l}\text { *Religious activity } \\
* \text { Not sure/missing }\end{array}$ & $\begin{array}{l}* \text { Other (religious activity, medical } \\
\text { appointment, babysitting, GUI } \\
\text { activity) } \\
\text { *Don't know/missing }\end{array}$ \\
\hline
\end{tabular}


Table A2 Diary characteristics by wave, weighted

\begin{tabular}{|c|c|c|c|c|c|}
\hline \multirow[b]{2}{*}{ Variable } & \multirow[b]{2}{*}{ Category } & \multicolumn{2}{|c|}{$\begin{array}{c}\text { Wave } 1 \\
(\mathrm{~N}=3923)\end{array}$} & \multicolumn{2}{|c|}{$\begin{array}{c}\text { Wave2 } \\
(\mathrm{N}=3257)\end{array}$} \\
\hline & & No. & $\%$ & No. & $\%$ \\
\hline \multirow[t]{5}{*}{ Day completed } & Monday & 886 & 23 & 668 & 22 \\
\hline & Tuesday & 853 & 22 & 635 & 21 \\
\hline & Wednesday & 745 & 19 & 606 & 20 \\
\hline & Thursday & 731 & 19 & 560 & 18 \\
\hline & Friday & 626 & 16 & 563 & 19 \\
\hline \multirow[t]{12}{*}{ Month completed } & January & 335 & 9 & 283 & 9 \\
\hline & February & 100 & 3 & 217 & 7 \\
\hline & March & 23 & 1 & 392 & 13 \\
\hline & April & 590 & 15 & 19 & 1 \\
\hline & May & 154 & 4 & 26 & 1 \\
\hline & June & 71 & 2 & 2 & 0 \\
\hline & July & 1 & 0 & 0 & 0 \\
\hline & August & 37 & 1 & 20 & 1 \\
\hline & September & 632 & 16 & 497 & 16 \\
\hline & October & 810 & 21 & 614 & 21 \\
\hline & November & 744 & 19 & 567 & 19 \\
\hline & December & 343 & 9 & 396 & 13 \\
\hline \multirow{5}{*}{$\begin{array}{l}\text { When was diary } \\
\text { completed? }\end{array}$} & Now and then during diary day & 1,153 & 30 & 569 & 19 \\
\hline & End of diary day & 1,311 & 34 & 1,201 & 40 \\
\hline & Day after diary day & 643 & 17 & 646 & 21 \\
\hline & Later & 315 & 8 & 290 & 10 \\
\hline & Don't know & 419 & 11 & 326 & 11 \\
\hline \multirow{3}{*}{$\begin{array}{l}\text { Who completed the } \\
\text { diary? }\end{array}$} & Child (or child helped) & 2,071 & 54 & 1,375 & 45 \\
\hline & Parent only & 1,369 & 36 & 1,337 & 44 \\
\hline & Don't know & 401 & 10 & 320 & 11 \\
\hline
\end{tabular}


Table A3 Average time spent in activities (minutes) by socio-demographic group for girls

\begin{tabular}{|c|c|c|c|c|c|c|c|c|c|c|}
\hline & \multicolumn{5}{|c|}{ Income Quintile } & \multicolumn{5}{|c|}{ Mother's Education } \\
\hline & & \multicolumn{2}{|c|}{ Wave 1} & \multicolumn{2}{|c|}{ Wave 2} & & \multicolumn{2}{|c|}{ Wave 1} & \multicolumn{2}{|c|}{ Wave 2} \\
\hline & & Mean & $95 \% \mathrm{Cl}$ & Mean & $95 \% \mathrm{Cl}$ & & Mean & $95 \% \mathrm{Cl}$ & Mean & $95 \% \mathrm{Cl}$ \\
\hline \multirow[t]{5}{*}{ Sleep } & Lowest & 676 & {$[661,692]$} & 552 & {$[544,560]$} & Less than Secondary & 671 & {$[661,681]$} & 548 & {$[539,558]$} \\
\hline & $2 n d$ & 659 & {$[651,667]$} & 547 & {$[539,554]$} & Secondary & 662 & {$[656,669]$} & 546 & {$[540,551]$} \\
\hline & $3 r d$ & 670 & {$[662,678]$} & 550 & {$[541,559]$} & More than secondary & 656 & {$[651,661]$} & 546 & {$[541,550]$} \\
\hline & 4 th & 660 & {$[654,666]$} & 545 & {$[537,554]$} & & & & & \\
\hline & Highest & 653 & {$[644,662]$} & 539 & {$[532,546]$} & & & & & \\
\hline \multirow[t]{5}{*}{ Homework } & Lowest & 51 & {$[45,57]$} & 76 & {$[66,86]$} & Less than Secondary & 50 & {$[46,54]$} & 81 & {$[67,95]$} \\
\hline & 2nd & 48 & {$[44,52]$} & 79 & {$[71,87]$} & Secondary & 48 & {$[45,51]$} & 83 & {$[78,89]$} \\
\hline & $3 r d$ & 49 & {$[44,53]$} & 88 & {$[75,100]$} & More than secondary & 45 & {$[42,47]$} & 81 & {$[76,86]$} \\
\hline & 4 th & 47 & {$[43,50]$} & 84 & {$[76,91]$} & & & & & \\
\hline & Highest & 43 & {$[40,47]$} & 78 & {$[72,84]$} & & & & & \\
\hline \multirow[t]{5}{*}{ Reading } & Lowest & 21 & {$[16,27]$} & 12 & {$[7,16]$} & Less than Secondary & 17 & {$[13,20]$} & 6 & {$[3,10]$} \\
\hline & $2 n d$ & 18 & {$[15,22]$} & 7 & {$[4,9]$} & Secondary & 21 & {$[18,23]$} & 9 & {$[7,11]$} \\
\hline & $3 r d$ & 21 & {$[18,24]$} & 8 & {$[5,11]$} & More than secondary & 23 & {$[21,25]$} & 14 & {$[11,17]$} \\
\hline & 4 th & 21 & {$[19,24]$} & 13 & {$[9,16]$} & & & & & \\
\hline & Highest & 20 & {$[17,23]$} & 14 & {$[9,20]$} & & & & & \\
\hline \multirow[t]{5}{*}{ Sport } & Lowest & 40 & {$[29,50]$} & 20 & {$[12,28]$} & Less than Secondary & 54 & {$[46,62]$} & 12 & {$[8,16]$} \\
\hline & $2 n d$ & 54 & {$[46,63]$} & 16 & {$[11,21]$} & Secondary & 49 & {$[44,54]$} & 20 & {$[15,24]$} \\
\hline & $3 r d$ & 45 & {$[39,51]$} & 18 & {$[14,23]$} & More than secondary & 46 & {$[42,50]$} & 27 & {$[23,31]$} \\
\hline & 4 th & 50 & {$[44,57]$} & 23 & {$[17,28]$} & & & & & \\
\hline & Highest & 54 & {$[47,61]$} & 28 & {$[22,34]$} & & & & & \\
\hline
\end{tabular}




\begin{tabular}{|c|c|c|c|c|c|c|c|c|c|c|}
\hline \multirow[t]{5}{*}{ Housework } & Lowest & 6 & {$[4,9]$} & 5 & {$[3,7]$} & Less than Secondary & 5 & {$[3,7]$} & 4 & {$[2,6]$} \\
\hline & 2nd & 8 & {$[5,10]$} & 7 & {$[4,9]$} & Secondary & 6 & {$[5,8]$} & 6 & {$[4,7]$} \\
\hline & $3 r d$ & 7 & {$[5,8]$} & 6 & {$[4,8]$} & More than secondary & 7 & {$[5,8]$} & 8 & {$[6,9]$} \\
\hline & 4th & 6 & {$[4,8]$} & 8 & {$[5,10]$} & & & & & \\
\hline & Highest & 4 & {$[3,5]$} & 6 & {$[5,8]$} & & & & & \\
\hline \multirow[t]{5}{*}{ Media } & Lowest & 68 & {$[58,79]$} & 94 & {$[80,109]$} & Less than Secondary & 70 & {$[63,77]$} & 108 & {$[90,125]$} \\
\hline & 2nd & 74 & {$[66,82]$} & 101 & {$[89,113]$} & Secondary & 72 & {$[67,78]$} & 103 & {$[94,111]$} \\
\hline & $3 r d$ & 72 & {$[65,80]$} & 99 & {$[87,112]$} & More than secondary & 67 & {$[63,71]$} & 90 & {$[82,97]$} \\
\hline & 4th & 71 & {$[65,76]$} & 99 & {$[89,109]$} & & & & & \\
\hline & Highest & 66 & {$[60,71]$} & 95 & {$[81,110]$} & & & & & \\
\hline \multirow[t]{5}{*}{ School } & Lowest & 343 & {$[327,359]$} & 420 & {$[411,429]$} & Less than Secondary & 344 & {$[335,354]$} & 416 & {$[404,428]$} \\
\hline & $2 n d$ & 341 & {$[334,348]$} & 431 & {$[421,441]$} & Secondary & 344 & {$[340,348]$} & 423 & {$[417,429]$} \\
\hline & $3 r d$ & 343 & {$[337,349]$} & 416 & {$[406,426]$} & More than secondary & 344 & {$[340,348]$} & 424 & {$[420,428]$} \\
\hline & 4th & 347 & {$[342,351]$} & 417 & {$[410,425]$} & & & & & \\
\hline & Highest & 344 & {$[337,351]$} & 425 & {$[418,433]$} & & & & & \\
\hline \multirow[t]{5}{*}{ Playing } & Lowest & 45 & {$[34,56]$} & 44 & {$[33,55]$} & Less than Secondary & 38 & {$[31,45]$} & 57 & {$[43,70]$} \\
\hline & $2 n d$ & 37 & {$[31,43]$} & 40 & {$[30,50]$} & Secondary & 41 & {$[37,46]$} & 36 & {$[30,42]$} \\
\hline & $3 r d$ & 37 & {$[32,43]$} & 46 & {$[35,57]$} & More than secondary & 39 & {$[36,43]$} & 33 & {$[27,39]$} \\
\hline & 4th & 43 & {$[37,48]$} & 34 & {$[26,41]$} & & & & & \\
\hline & Highest & 42 & {$[37,47]$} & 32 & {$[22,42]$} & & & & & \\
\hline \multirow[t]{5}{*}{ Leisure } & Lowest & 18 & {$[12,24]$} & 12 & {$[7,17]$} & Less than Secondary & 13 & {$[9,17]$} & 19 & {$[10,28]$} \\
\hline & 2nd & 17 & {$[12,22]$} & 16 & {$[11,22]$} & Secondary & 17 & {$[13,20]$} & 14 & {$[10,18]$} \\
\hline & $3 r d$ & 13 & {$[10,17]$} & 20 & {$[11,28]$} & More than secondary & 21 & {$[18,24]$} & 19 & {$[16,22]$} \\
\hline & 4 th & 17 & {$[14,21]$} & 17 & {$[12,21]$} & & & & & \\
\hline & Highest & 20 & {$[16,24]$} & 18 & {$[14,21]$} & & & & & \\
\hline
\end{tabular}




\begin{tabular}{|c|c|c|c|c|c|c|c|c|c|c|}
\hline \multirow[t]{5}{*}{$\begin{array}{l}\text { Personal } \\
\text { Care }\end{array}$} & Lowest & 135 & {$[123,148]$} & 143 & {$[132,155]$} & Less than Secondary & 138 & {$[130,146]$} & 140 & {$[128,152]$} \\
\hline & $2 n d$ & 140 & {$[132,149]$} & 140 & {$[131,150]$} & Secondary & 137 & {$[133,142]$} & 141 & {$[135,146]$} \\
\hline & $3 r d$ & 140 & {$[134,146]$} & 138 & {$[130,145]$} & More than secondary & 157 & {$[150,164]$} & 146 & {$[140,151]$} \\
\hline & 4 th & 146 & {$[140,152]$} & 142 & {$[134,150]$} & & & & & \\
\hline & Highest & 156 & {$[144,169]$} & 150 & {$[142,159]$} & & & & & \\
\hline \multirow[t]{5}{*}{ Family } & Lowest & 13 & {$[7,19]$} & 47 & {$[37,57]$} & Less than Secondary & 14 & {$[9,18]$} & 41 & {$[32,51]$} \\
\hline & $2 n d$ & 15 & {$[10,20]$} & 45 & {$[36,54]$} & Secondary & 18 & {$[14,22]$} & 50 & {$[44,56]$} \\
\hline & $3 r d$ & 19 & {$[14,24]$} & 44 & {$[35,53]$} & More than secondary & 17 & {$[12,22]$} & 43 & {$[38,48]$} \\
\hline & 4 th & 15 & {$[11,20]$} & 48 & {$[41,56]$} & & & & & \\
\hline & Highest & 20 & {$[11,28]$} & 44 & {$[37,52]$} & & & & & \\
\hline \multirow[t]{5}{*}{ Other } & Lowest & 22 & {$[15,29]$} & 14 & {$[8,19]$} & Less than Secondary & 25 & {$[19,32]$} & 8 & {$[4,12]$} \\
\hline & 2nd & 29 & {$[20,38]$} & 11 & {$[6,15]$} & Secondary & 24 & {$[19,29]$} & 10 & {$[7,13]$} \\
\hline & $3 r d$ & 23 & {$[18,28]$} & 6 & {$[4,9]$} & More than secondary & 19 & {$[16,22]$} & 10 & {$[8,13]$} \\
\hline & 4 th & 17 & {$[13,21]$} & 11 & {$[6,17]$} & & & & & \\
\hline & Highest & 19 & {$[14,23]$} & 9 & {$[6,12]$} & & & & & \\
\hline
\end{tabular}


Table A4 Average time spent in activities (minutes) by socio-demographic group for boys

\begin{tabular}{|c|c|c|c|c|c|c|c|c|c|c|}
\hline & \multicolumn{5}{|c|}{ Income Quintile } & \multicolumn{5}{|c|}{ Mother's Education } \\
\hline & & \multicolumn{2}{|r|}{ Wave 1} & \multicolumn{2}{|c|}{ Wave 2} & & \multicolumn{2}{|c|}{ Wave 1} & \multicolumn{2}{|c|}{ Wave 2} \\
\hline & & Mean & $95 \% \mathrm{Cl}$ & Mean & $95 \% \mathrm{Cl}$ & & Mean & $95 \% \mathrm{Cl}$ & Mean & $95 \% \mathrm{Cl}$ \\
\hline \multirow[t]{5}{*}{ Sleep } & Lowest & 661 & {$[649,673]$} & 553 & {$[545,561]$} & Less than Secondary & 658 & {$[649,667]$} & 552 & {$[542,562]$} \\
\hline & 2nd & 659 & {$[652,667]$} & 550 & {$[537,562]$} & Secondary & 664 & {$[659,669]$} & 552 & {$[546,559]$} \\
\hline & $3 r d$ & 662 & {$[654,671]$} & 555 & {$[548,562]$} & More than secondary & 650 & {$[646,655]$} & 549 & {$[546,553]$} \\
\hline & 4th & 654 & {$[648,660]$} & 551 & {$[544,557]$} & & & & & \\
\hline & Highest & 651 & {$[645,657]$} & 546 & {$[540,552]$} & & & & & \\
\hline \multirow[t]{5}{*}{ Homework } & Lowest & 44 & {$[38,51]$} & 70 & {$[62,78]$} & Less than Secondary & 49 & {$[44,53]$} & 62 & {$[54,70]$} \\
\hline & 2nd & 50 & {$[45,55]$} & 65 & {$[56,73]$} & Secondary & 46 & {$[43,49]$} & 73 & {$[67,79]$} \\
\hline & $3 r d$ & 42 & {$[38,46]$} & 76 & {$[70,82]$} & More than secondary & 42 & {$[39,44]$} & 73 & {$[69,78]$} \\
\hline & 4 th & 48 & {$[44,52]$} & 69 & {$[63,76]$} & & & & & \\
\hline & Highest & 44 & {$[40,47]$} & 75 & {$[68,83]$} & & & & & \\
\hline \multirow[t]{5}{*}{ Reading } & Lowest & 12 & {$[7,16]$} & 6 & {$[4,9]$} & Less than Secondary & 12 & {$[9,15]$} & 5 & {$[3,8]$} \\
\hline & 2nd & 21 & {$[17,24]$} & 13 & {$[7,18]$} & Secondary & 17 & {$[15,19]$} & 7 & {$[5,10]$} \\
\hline & $3 r d$ & 16 & {$[13,19]$} & 6 & {$[4,9]$} & More than secondary & 24 & {$[21,27]$} & 12 & {$[10,15]$} \\
\hline & 4 th & 17 & {$[15,20]$} & 8 & {$[6,11]$} & & & & & \\
\hline & Highest & 19 & {$[16,21]$} & 12 & {$[8,16]$} & & & & & \\
\hline \multirow[t]{5}{*}{ Sport } & Lowest & 75 & {$[60,90]$} & 31 & {$[23,39]$} & Less than Secondary & 75 & {$[64,85]$} & 31 & {$[22,40]$} \\
\hline & 2nd & 65 & {$[56,75]$} & 21 & {$[15,27]$} & Secondary & 71 & {$[65,77]$} & 48 & {$[27,68]$} \\
\hline & $3 r d$ & 72 & {$[64,80]$} & 32 & {$[25,39]$} & More than secondary & 68 & {$[64,73]$} & 37 & {$[33,42]$} \\
\hline & 4 th & 73 & {$[64,82]$} & 38 & {$[31,45]$} & & & & & \\
\hline & Highest & 72 & {$[67,78]$} & 54 & {$[42,65]$} & & & & & \\
\hline
\end{tabular}




\begin{tabular}{|c|c|c|c|c|c|c|c|c|c|c|}
\hline \multirow[t]{5}{*}{ Housework } & Lowest & 5 & {$[2,7]$} & 5 & {$[3,8]$} & Less than Secondary & 5 & {$[3,7]$} & 6 & {$[2,9]$} \\
\hline & 2nd & 7 & {$[3,12]$} & 7 & {$[4,10]$} & Secondary & 7 & {$[4,9]$} & 4 & {$[3,6]$} \\
\hline & $3 r d$ & 6 & {$[4,9]$} & 5 & {$[3,7]$} & More than secondary & 6 & {$[5,7]$} & 5 & {$[3,6]$} \\
\hline & 4 th & 6 & {$[5,8]$} & 3 & {$[2,5]$} & & & & & \\
\hline & Highest & 5 & {$[4,7]$} & 4 & {$[2,5]$} & & & & & \\
\hline \multirow[t]{5}{*}{ Media } & Lowest & 80 & {$[69,92]$} & 107 & {$[93,121]$} & Less than Secondary & 83 & {$[75,91]$} & 120 & {$[104,136]$} \\
\hline & 2nd & 79 & {$[71,88]$} & 125 & {$[109,141]$} & Secondary & 80 & {$[75,85]$} & 107 & {$[98,117]$} \\
\hline & $3 r d$ & 79 & {$[72,86]$} & 105 & {$[94,117]$} & More than secondary & 79 & {$[74,84]$} & 104 & {$[96,111]$} \\
\hline & 4 th & 83 & {$[76,91]$} & 114 & {$[102,127]$} & & & & & \\
\hline & Highest & 82 & {$[76,89]$} & 99 & {$[87,110]$} & & & & & \\
\hline \multirow[t]{5}{*}{ School } & Lowest & 352 & {$[345,360]$} & 418 & {$[411,426]$} & Less than Secondary & 347 & {$[340,355]$} & 413 & {$[399,426]$} \\
\hline & 2nd & 347 & {$[342,352]$} & 414 & {$[401,428]$} & Secondary & 341 & {$[337,345]$} & 404 & {$[389,419]$} \\
\hline & $3 r d$ & 341 & {$[332,349]$} & 413 & {$[402,424]$} & More than secondary & 348 & {$[344,352]$} & 418 & {$[412,424]$} \\
\hline & 4 th & 340 & {$[333,347]$} & 409 & {$[399,419]$} & & & & & \\
\hline & Highest & 349 & {$[343,355]$} & 415 & {$[401,428]$} & & & & & \\
\hline \multirow[t]{5}{*}{ Playing } & Lowest & 33 & {$[22,44]$} & 43 & {$[33,54]$} & Less than Secondary & 27 & {$[20,35]$} & 47 & {$[35,59]$} \\
\hline & 2nd & 28 & {$[21,35]$} & 41 & {$[30,53]$} & Secondary & 28 & {$[24,33]$} & 40 & {$[32,48]$} \\
\hline & $3 r d$ & 29 & {$[23,34]$} & 38 & {$[29,48]$} & More than secondary & 30 & {$[27,33]$} & 32 & {$[27,37]$} \\
\hline & 4 th & 30 & {$[25,35]$} & 37 & {$[29,45]$} & & & & & \\
\hline & Highest & 27 & {$[23,31]$} & 31 & {$[20,43]$} & & & & & \\
\hline \multirow[t]{5}{*}{ Leisure } & Lowest & 6 & {$[3,10]$} & 13 & {$[7,19]$} & Less than Secondary & 8 & {$[5,12]$} & 9 & {$[5,14]$} \\
\hline & 2nd & 8 & {$[4,12]$} & 17 & {$[10,23]$} & Secondary & 9 & {$[7,11]$} & 15 & {$[11,18]$} \\
\hline & $3 r d$ & 10 & {$[7,14]$} & 13 & {$[8,17]$} & More than secondary & 13 & {$[11,15]$} & 15 & {$[12,17]$} \\
\hline & 4 th & 12 & {$[8,15]$} & 15 & {$[11,19]$} & & & & & \\
\hline & Highest & 13 & {$[11,16]$} & 12 & {$[9,15]$} & & & & & \\
\hline
\end{tabular}




\begin{tabular}{|c|c|c|c|c|c|c|c|c|c|c|}
\hline \multirow[t]{5}{*}{$\begin{array}{l}\text { Personal } \\
\text { Care }\end{array}$} & Lowest & 126 & {$[116,136]$} & 130 & {$[122,138]$} & Less than Secondary & 131 & {$[123,138]$} & 123 & {$[114,133]$} \\
\hline & 2nd & 138 & {$[131,145]$} & 130 & {$[120,141]$} & Secondary & 139 & {$[134,143]$} & 130 & {$[125,136]$} \\
\hline & $3 r d$ & 141 & {$[134,149]$} & 127 & {$[118,136]$} & More than secondary & 147 & {$[143,152]$} & 140 & {$[135,145]$} \\
\hline & 4th & 139 & {$[134,144]$} & 137 & {$[129,144]$} & & & & & \\
\hline & Highest & 148 & {$[142,154]$} & 143 & {$[136,149]$} & & & & & \\
\hline \multirow[t]{5}{*}{ Family } & Lowest & 17 & {$[6,27]$} & 48 & {$[37,58]$} & Less than Secondary & 17 & {$[9,26]$} & 58 & {$[45,70]$} \\
\hline & 2nd & 20 & {$[8,32]$} & 46 & {$[35,56]$} & Secondary & 16 & {$[11,20]$} & 48 & {$[41,56]$} \\
\hline & $3 r d$ & 17 & {$[12,22]$} & 57 & {$[44,69]$} & More than secondary & 14 & {$[10,18]$} & 45 & {$[39,50]$} \\
\hline & 4 th & 14 & {$[10,19]$} & 49 & {$[41,57]$} & & & & & \\
\hline & Highest & 12 & {$[9,15]$} & 41 & {$[32,50]$} & & & & & \\
\hline \multirow[t]{5}{*}{ Other } & Lowest & 28 & {$[19,38]$} & 15 & {$[8,22]$} & Less than Secondary & 28 & {$[21,35]$} & 15 & {$[8,22]$} \\
\hline & 2nd & 18 & {$[13,22]$} & 11 & {$[4,18]$} & Secondary & 23 & {$[18,27]$} & 11 & {$[8,14]$} \\
\hline & $3 r d$ & 25 & {$[19,30]$} & 12 & {$[8,17]$} & More than secondary & 18 & {$[15,21]$} & 10 & {$[7,13]$} \\
\hline & 4 th & 23 & {$[17,30]$} & 10 & {$[6,14]$} & & & & & \\
\hline & Highest & 17 & {$[12,21]$} & 8 & {$[5,11]$} & & & & & \\
\hline
\end{tabular}


Table A5 Average substitution patterns (in minutes) from wave 1 activities to wave 2 activities

\begin{tabular}{|c|c|c|c|c|c|c|c|c|c|c|c|c|}
\hline & Sleeping & School & Per. Care & Sport & Playing & Media & Reading & Family & Homework & Housework & Leisure & Other \\
\hline Sleeping & & 5 & 38 & 2 & 4 & 31 & 6 & 10 & 5 & 1 & 3 & 1 \\
\hline \multicolumn{13}{|l|}{ School } \\
\hline Per. Care & & 41 & & & & 3 & & 4 & 4 & & & \\
\hline Sport & & 6 & 4 & . & 3 & 3 & & 4 & 12 & & & \\
\hline Playing & & 2 & & & & & & 1 & 6 & & & \\
\hline Media & & 3 & & & 1 & 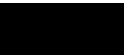 & & 7 & 10 & & 1 & \\
\hline Reading & & & 2 & 1 & 1 & 6 & & 2 & 2 & & 1 & \\
\hline Family & & 1 & & & & & & & & & & \\
\hline Homework & & 10 & & & & & & & & & & \\
\hline Housework & & & & & & & & & 1 & & & \\
\hline Leisure & & 1 & & & & & & 1 & 2 & & & \\
\hline Other & & 3 & 1 & & 1 & 1 & & 2 & 3 & & & \\
\hline
\end{tabular}

Notes: Rows correspond to wave 1 activities and columns correspond to wave 2 activities. Results are averaged across substitutions by the same child and in the same timeslot, and the net amount for any two activities is shown. "Per. Care" = Personal Care. Results are for girls and boys combined. 
Table A6 Results for participation for girls (Full table)

\begin{tabular}{|c|c|c|c|c|c|c|c|c|c|c|c|c|c|c|}
\hline \multirow[b]{2}{*}{ Variables } & \multicolumn{7}{|c|}{ Linear regression } & \multicolumn{7}{|c|}{ Logit OR } \\
\hline & $\begin{array}{l}\text { Any } \\
\text { Sport }\end{array}$ & $\begin{array}{c}\text { Any } \\
\text { Reading }\end{array}$ & $\begin{array}{c}\text { Any } \\
\text { Homework }\end{array}$ & $\begin{array}{l}\text { Any } \\
\text { Playing }\end{array}$ & $\begin{array}{l}\text { Any } \\
\text { Media }\end{array}$ & $\begin{array}{c}\text { Any } \\
\text { Hobbies }\end{array}$ & $\begin{array}{l}\text { Any } \\
\text { Family }\end{array}$ & $\begin{array}{l}\text { Any } \\
\text { Sport }\end{array}$ & $\begin{array}{c}\text { Any } \\
\text { Reading }\end{array}$ & $\begin{array}{c}\text { Any } \\
\text { Homework }\end{array}$ & $\begin{array}{c}\text { Any } \\
\text { Playing }\end{array}$ & $\begin{array}{l}\text { Any } \\
\text { Media }\end{array}$ & $\begin{array}{c}\text { Any } \\
\text { Hobbies }\end{array}$ & $\begin{array}{l}\text { Any } \\
\text { Family }\end{array}$ \\
\hline \multirow{3}{*}{\multicolumn{15}{|c|}{$\begin{array}{l}\text { Mother's Education: } \\
\text { Omitted=Less than } \\
\text { Secondary }\end{array}$}} \\
\hline & & & & & & & & & & & & & & \\
\hline & & & & & & & & & & & & & & \\
\hline \multirow[t]{2}{*}{ Secondary } & -0.01 & $0.11 * *$ & -0.00 & 0.07 & 0.04 & 0.05 & $0.06^{*}$ & 0.95 & $1.56^{* *}$ & 0.97 & 1.33 & 1.40 & 1.28 & 1.43 \\
\hline & $(0.04)$ & $(0.04)$ & $(0.03)$ & $(0.04)$ & $(0.03)$ & $(0.04)$ & $(0.03)$ & $(0.16)$ & $(0.27)$ & $(0.22)$ & $(0.22)$ & $(0.32)$ & $(0.26)$ & $(0.28)$ \\
\hline \multirow[t]{2}{*}{ More than Secondary } & -0.03 & $0.17 * * *$ & -0.01 & 0.04 & 0.01 & $0.14 * * *$ & 0.04 & 0.89 & $2.05^{* * *}$ & 0.92 & 1.19 & 1.08 & $1.96^{* * *}$ & 1.29 \\
\hline & $(0.04)$ & $(0.04)$ & $(0.02)$ & $(0.04)$ & $(0.03)$ & $(0.04)$ & $(0.03)$ & $(0.15)$ & $(0.34)$ & $(0.20)$ & $(0.19)$ & $(0.22)$ & $(0.36)$ & $(0.25)$ \\
\hline \multirow[t]{2}{*}{ Wave $=2$} & $-0.37 * * *$ & $-0.26 * * *$ & 0.07 & 0.01 & 0.03 & -0.01 & $0.34 * * *$ & $0.19 * * *$ & $0.24 * * *$ & 1.91 & 1.04 & 1.22 & 0.92 & $4.97^{* * *}$ \\
\hline & $(0.05)$ & $(0.05)$ & $(0.04)$ & $(0.06)$ & $(0.05)$ & $(0.05)$ & $(0.06)$ & $(0.05)$ & $(0.08)$ & $(0.87)$ & $(0.26)$ & $(0.41)$ & $(0.28)$ & $(1.35)$ \\
\hline \multirow[t]{2}{*}{ Secondary * Wave 2} & 0.04 & -0.04 & -0.03 & -0.15 & -0.03 & -0.06 & 0.02 & 1.20 & 1.04 & 0.71 & 0.54 & 0.77 & 0.73 & 0.96 \\
\hline & $(0.06)$ & $(0.06)$ & $(0.05)$ & $(0.07)$ & $(0.05)$ & $(0.06)$ & $(0.07)$ & $(0.38)$ & $(0.40)$ & $(0.35)$ & $(0.16)$ & $(0.31)$ & $(0.24)$ & $(0.30)$ \\
\hline \multicolumn{15}{|l|}{ More than Secondary * } \\
\hline \multirow[t]{2}{*}{ Wave 2} & $0.15^{*}$ & -0.01 & -0.03 & -0.13 & -0.03 & -0.06 & -0.02 & 2.05 & 1.36 & 0.76 & 0.59 & 0.82 & 0.78 & 0.86 \\
\hline & $(0.06)$ & $(0.06)$ & $(0.04)$ & $(0.07)$ & $(0.05)$ & $(0.06)$ & $(0.06)$ & $(0.62)$ & $(0.51)$ & $(0.36)$ & $(0.16)$ & $(0.30)$ & $(0.24)$ & $(0.27)$ \\
\hline \multicolumn{15}{|l|}{$\begin{array}{l}\text { Day Diary Completed: } \\
\text { Omitted=Monday }\end{array}$} \\
\hline \multirow[t]{2}{*}{ Tuesday } & $0.08^{* *}$ & -0.02 & -0.01 & -0.02 & -0.00 & -0.02 & -0.03 & $1.47^{* *}$ & 0.91 & 0.82 & 0.92 & 0.97 & 0.89 & 0.84 \\
\hline & $(0.03)$ & $(0.03)$ & $(0.02)$ & $(0.04)$ & $(0.03)$ & $(0.03)$ & $(0.03)$ & $(0.21)$ & $(0.14)$ & $(0.23)$ & $(0.14)$ & $(0.18)$ & $(0.14)$ & $(0.13)$ \\
\hline \multirow[t]{2}{*}{ Wednesday } & 0.03 & 0.01 & -0.04 & -0.06 & 0.02 & 0.01 & $-0.09 * *$ & 1.16 & 1.05 & 0.60 & 0.79 & 1.15 & 1.05 & $0.63^{* *}$ \\
\hline & $(0.03)$ & $(0.03)$ & $(0.02)$ & $(0.04)$ & $(0.03)$ & $(0.03)$ & $(0.03)$ & $(0.19)$ & $(0.17)$ & $(0.17)$ & $(0.12)$ & $(0.22)$ & $(0.17)$ & $(0.10)$ \\
\hline Thursday & 0.03 & -0.04 & -0.03 & 0.01 & 0.01 & -0.03 & -0.04 & 1.15 & 0.82 & 0.64 & 1.03 & 1.11 & 0.85 & 0.82 \\
\hline
\end{tabular}




\begin{tabular}{|c|c|c|c|c|c|c|c|c|c|c|c|c|c|c|}
\hline & $(0.03)$ & $(0.04)$ & $(0.02)$ & $(0.04)$ & $(0.02)$ & $(0.03)$ & $(0.03)$ & $(0.17)$ & $(0.14)$ & $(0.16)$ & $(0.16)$ & $(0.20)$ & $(0.14)$ & (0.13) \\
\hline Friday & $\begin{array}{c}0.07 \\
(0.03)\end{array}$ & $\begin{array}{l}-0.05 \\
(0.04)\end{array}$ & $\begin{array}{c}-0.41^{* * *} \\
(0.03)\end{array}$ & $\begin{array}{c}0.03 \\
(0.04)\end{array}$ & $\begin{array}{c}0.02 \\
(0.03)\end{array}$ & $\begin{array}{l}-0.06 \\
(0.03)\end{array}$ & $\begin{array}{c}0.07 \\
(0.03)\end{array}$ & $\begin{array}{c}1.40 \\
(0.23)\end{array}$ & $\begin{array}{c}0.78 \\
(0.13)\end{array}$ & $\begin{array}{c}0.07^{* * *} \\
(0.02)\end{array}$ & $\begin{array}{c}1.13 \\
(0.18)\end{array}$ & $\begin{array}{c}1.18 \\
(0.26)\end{array}$ & $\begin{array}{c}0.74 \\
(0.12)\end{array}$ & $\begin{array}{c}1.43 \\
(0.23)\end{array}$ \\
\hline \multicolumn{15}{|c|}{$\begin{array}{l}\text { Month Diary Completed: } \\
\text { Omitted=January }\end{array}$} \\
\hline \multirow[t]{2}{*}{ February } & 0.08 & -0.11 & 0.04 & -0.03 & 0.02 & 0.07 & -0.05 & 1.44 & 0.57 & 1.51 & 0.90 & 1.22 & 1.44 & 0.82 \\
\hline & $(0.05)$ & $(0.06)$ & $(0.04)$ & $(0.06)$ & $(0.04)$ & $(0.06)$ & $(0.06)$ & (0.39) & $(0.17)$ & $(0.56)$ & $(0.23)$ & $(0.44)$ & $(0.42)$ & $(0.23)$ \\
\hline \multirow[t]{2}{*}{ March } & $0.19 * *$ & -0.00 & $0.06^{*}$ & 0.03 & 0.01 & 0.00 & 0.07 & $2.42 * *$ & 1.03 & 1.88 & 1.15 & 1.07 & 0.98 & 1.39 \\
\hline & $(0.06)$ & $(0.07)$ & $(0.04)$ & $(0.07)$ & $(0.04)$ & $(0.05)$ & $(0.06)$ & $(0.69)$ & $(0.37)$ & $(0.69)$ & $(0.32)$ & $(0.41)$ & $(0.29)$ & $(0.40)$ \\
\hline \multirow[t]{2}{*}{ April } & $0.20 * * *$ & -0.07 & 0.03 & -0.04 & -0.00 & 0.00 & 0.01 & $2.38 * * *$ & 0.73 & 1.39 & 0.85 & 0.96 & 1.01 & 1.01 \\
\hline & $(0.06)$ & $(0.06)$ & $(0.04)$ & $(0.06)$ & $(0.04)$ & $(0.05)$ & $(0.05)$ & $(0.63)$ & $(0.20)$ & $(0.42)$ & $(0.21)$ & $(0.33)$ & $(0.28)$ & $(0.27)$ \\
\hline \multirow[t]{2}{*}{ May } & $0.20 *$ & -0.11 & -0.00 & -0.17 & -0.07 & 0.06 & 0.11 & 2.45 & 0.59 & 1.03 & 0.49 & 0.58 & 1.35 & 1.73 \\
\hline & $(0.08)$ & $(0.08)$ & $(0.05)$ & $(0.08)$ & $(0.06)$ & $(0.07)$ & $(0.07)$ & $(0.93)$ & $(0.23)$ & $(0.47)$ & $(0.17)$ & $(0.26)$ & $(0.49)$ & (0.65) \\
\hline \multirow[t]{2}{*}{ June } & $0.37^{* * *}$ & 0.03 & -0.00 & 0.05 & -0.04 & -0.05 & -0.06 & $6.25 * * *$ & 1.12 & 1.08 & 1.24 & 0.74 & 0.76 & 0.66 \\
\hline & $(0.07)$ & $(0.10)$ & $(0.08)$ & $(0.09)$ & $(0.07)$ & $(0.07)$ & $(0.07)$ & (2.89) & $(0.50)$ & $(0.62)$ & $(0.50)$ & $(0.37)$ & $(0.32)$ & $(0.33)$ \\
\hline \multicolumn{15}{|l|}{ July } \\
\hline \multirow[t]{2}{*}{ August } & 0.13 & -0.09 & 0.07 & 0.04 & 0.00 & $-0.17 * * *$ & 0.11 & 1.75 & 0.66 & 1.84 & 1.18 & 0.99 & $0.17^{* *}$ & 1.74 \\
\hline & $(0.14)$ & $(0.14)$ & $(0.10)$ & $(0.16)$ & $(0.09)$ & $(0.05)$ & $(0.14)$ & (1.08) & $(0.44)$ & $(1.28)$ & $(0.76)$ & $(0.77)$ & $(0.11)$ & (1.14) \\
\hline \multirow[t]{2}{*}{ September } & 0.09 & -0.08 & 0.07 & 0.00 & -0.02 & 0.01 & 0.00 & 1.55 & 0.67 & 1.96 & 1.00 & 0.85 & 1.07 & 1.02 \\
\hline & $(0.05)$ & $(0.06)$ & $(0.03)$ & $(0.05)$ & $(0.04)$ & $(0.04)$ & $(0.05)$ & $(0.34)$ & $(0.18)$ & $(0.57)$ & $(0.21)$ & $(0.26)$ & $(0.26)$ & $(0.25)$ \\
\hline \multirow[t]{2}{*}{ October } & $0.15^{* * *}$ & -0.04 & 0.02 & -0.03 & -0.03 & 0.04 & 0.05 & $2.01 * * *$ & 0.81 & 1.23 & 0.89 & 0.77 & 1.22 & 1.32 \\
\hline & $(0.05)$ & $(0.06)$ & $(0.03)$ & $(0.05)$ & $(0.04)$ & $(0.04)$ & $(0.04)$ & $(0.44)$ & $(0.22)$ & $(0.35)$ & $(0.19)$ & $(0.23)$ & $(0.29)$ & (0.29) \\
\hline \multirow[t]{2}{*}{ November } & 0.05 & -0.05 & 0.04 & -0.03 & -0.04 & 0.09 & 0.05 & 1.25 & 0.80 & 1.51 & 0.89 & 0.76 & 1.55 & 1.29 \\
\hline & $(0.05)$ & $(0.06)$ & (0.03) & $(0.05)$ & $(0.04)$ & (0.04) & $(0.04)$ & $(0.27)$ & $(0.21)$ & $(0.43)$ & $(0.18)$ & $(0.23)$ & $(0.35)$ & (0.29) \\
\hline \multirow[t]{2}{*}{ December } & 0.01 & -0.10 & -0.02 & 0.04 & -0.02 & 0.02 & 0.09 & 1.07 & 0.61 & 0.86 & 1.17 & 0.87 & 1.12 & 1.54 \\
\hline & $(0.05)$ & $(0.06)$ & $(0.04)$ & $(0.06)$ & $(0.04)$ & $(0.05)$ & $(0.05)$ & $(0.26)$ & $(0.18)$ & $(0.28)$ & $(0.27)$ & $(0.28)$ & $(0.29)$ & $(0.37)$ \\
\hline
\end{tabular}


Time Diary Completed:

Omitted=During Day

End of diary day

$\begin{array}{ccccccc}-0.03 & -0.02 & 0.01 & 0.02 & -0.01 & -0.05 & 0.01 \\ (0.03) & (0.03) & (0.02) & (0.03) & (0.02) & (0.03) & (0.03)\end{array}$

Day after diary day

$$
0.00
$$

(0.03)

$-0.05$

(0.04)$$
0.03
$$

0.03

(0.03)

$-0.08^{*}$

(0.03)

0.89

(0.11)

\subsection{7}

1.07

0.90

$0.79 \quad 1.04$

Later

Don't know

$\begin{array}{lllll}(0.02) & (0.04) & (0.03) & (0.03) & (0.03)\end{array}$

1.00
$(0.16)$

(0.16)

1.13

$(0.22)$

(0.13)

(0.15) (0.11) (0.14)

$\begin{array}{llllll}0.01 & -0.02 & 0.01 & -0.01 & -0.03 & -0.01\end{array}$

0.79

(0.17)

(0.28)

$(0.17)$

$0.58^{* *} \quad 0.89 \quad 0.91$

$\begin{array}{lllllll}0.65 & 0.89 & 3.35 * * * & 0.99 & 1.39 & 0.81 & 0.51\end{array}$

$\begin{array}{lllllll}(0.23) & (0.33) & (1.18) & (0.32) & (0.50) & (0.28) & (0.18)\end{array}$

Was Diary Completed with

Child? Omitted=Yes

\begin{tabular}{|c|c|c|c|c|c|c|c|c|c|c|c|c|c|c|}
\hline \multirow[t]{2}{*}{ Parent only } & 0.05 & -0.01 & 0.01 & 0.01 & -0.03 & -0.03 & 0.01 & 1.27 & 0.94 & 1.09 & 1.03 & 0.77 & 0.84 & 1.08 \\
\hline & $(0.02)$ & $(0.02)$ & $(0.02)$ & $(0.02)$ & $(0.02)$ & $(0.02)$ & $(0.02)$ & $(0.14)$ & $(0.10)$ & $(0.17)$ & $(0.10)$ & $(0.10)$ & $(0.10)$ & $(0.12)$ \\
\hline \multirow[t]{2}{*}{ Don't know } & 0.09 & 0.08 & $-0.13 * *$ & 0.10 & -0.11 & -0.01 & $0.16^{*}$ & 1.48 & 1.40 & $0.35^{* *}$ & 1.54 & 0.45 & 0.95 & $2.39 *$ \\
\hline & $(0.08)$ & $(0.10)$ & $(0.04)$ & $(0.08)$ & $(0.05)$ & $(0.07)$ & $(0.06)$ & $(0.55)$ & $(0.60)$ & $(0.12)$ & $(0.53)$ & $(0.15)$ & $(0.32)$ & $(0.83)$ \\
\hline \multirow{2}{*}{ Constant } & $0.46 * * *$ & $0.47 * * *$ & $0.88 * * *$ & $0.51 * * *$ & $0.87 * * *$ & $0.27 * * *$ & $0.16 * * *$ & 0.78 & 0.94 & $8.58 * * *$ & 1.04 & $6.87 * * *$ & $0.36 * * *$ & $0.19 * * *$ \\
\hline & $(0.06)$ & $(0.06)$ & $(0.04)$ & $(0.06)$ & $(0.04)$ & $(0.05)$ & $(0.05)$ & $(0.21)$ & $(0.27)$ & $(3.23)$ & $(0.26)$ & $(2.37)$ & $(0.10)$ & $(0.06)$ \\
\hline Observations & 3,606 & 3,606 & 3,606 & 3,606 & 3,606 & 3,606 & 3,606 & 3,606 & 3,606 & 3,606 & 3,606 & 3,606 & 3,606 & 3,606 \\
\hline R-Squared & 0.13 & 0.10 & 0.18 & 0.02 & 0.01 & 0.03 & 0.15 & & & & & & & \\
\hline
\end{tabular}

Notes: Clustered errors in parentheses. P-value alpha values of $0.01,0.05$, and 0.1 adjusted for multiple hypothesis testing using D/AP procedure according to the Bonferroni test with correlation $=0.15$ resulting in $p$-value cut-offs: ${ }^{* *} p<0.0016,{ }^{* *} p<0.008,{ }^{*} p<0.016$ 
Table A7 Results for participation for boys (Full table)

\begin{tabular}{|c|c|c|c|c|c|c|c|c|c|c|c|c|c|c|}
\hline \multirow[b]{2}{*}{ Variables } & \multicolumn{7}{|c|}{ Linear regression } & \multicolumn{7}{|c|}{ Logit } \\
\hline & $\begin{array}{l}\text { Any } \\
\text { Sport }\end{array}$ & $\begin{array}{c}\text { Any } \\
\text { Reading }\end{array}$ & $\begin{array}{c}\text { Any } \\
\text { Homework }\end{array}$ & $\begin{array}{c}\text { Any } \\
\text { Playing }\end{array}$ & $\begin{array}{c}\text { Any } \\
\text { Media }\end{array}$ & $\begin{array}{c}\text { Any } \\
\text { Hobbies }\end{array}$ & $\begin{array}{c}\text { Any } \\
\text { Family }\end{array}$ & $\begin{array}{l}\text { Any } \\
\text { Sport }\end{array}$ & $\begin{array}{c}\text { Any } \\
\text { Reading }\end{array}$ & $\begin{array}{c}\text { Any } \\
\text { Homework }\end{array}$ & $\begin{array}{l}\text { Any } \\
\text { Playing }\end{array}$ & $\begin{array}{l}\text { Any } \\
\text { Media }\end{array}$ & $\begin{array}{c}\text { Any } \\
\text { Hobbies }\end{array}$ & $\begin{array}{l}\text { Any } \\
\text { Family }\end{array}$ \\
\hline \multicolumn{15}{|l|}{$\begin{array}{l}\text { Mother's Education: } \\
\text { Omitted=Less than } \\
\text { Secondary }\end{array}$} \\
\hline Secondary & $\begin{array}{c}0.03 \\
(0.04)\end{array}$ & $\begin{array}{c}0.11^{* *} \\
(0.04)\end{array}$ & $\begin{array}{l}-0.03 \\
(0.03)\end{array}$ & $\begin{array}{c}0.04 \\
(0.04)\end{array}$ & $\begin{array}{c}0.02 \\
(0.03)\end{array}$ & $\begin{array}{c}0.05 \\
(0.03)\end{array}$ & $\begin{array}{c}0.00 \\
(0.04)\end{array}$ & $\begin{array}{c}1.14 \\
(0.21)\end{array}$ & $\begin{array}{l}1.61^{* *} \\
(0.28)\end{array}$ & $\begin{array}{c}0.79 \\
(0.21)\end{array}$ & $\begin{array}{c}1.19 \\
(0.21)\end{array}$ & $\begin{array}{c}1.17 \\
(0.29)\end{array}$ & $\begin{array}{c}1.46 \\
(0.34)\end{array}$ & $\begin{array}{c}1.03 \\
(0.23)\end{array}$ \\
\hline More than Secondary & $\begin{array}{c}0.05 \\
(0.03)\end{array}$ & $\begin{array}{c}0.25^{* * *} \\
(0.04)\end{array}$ & $\begin{array}{l}-0.07 \\
(0.03)\end{array}$ & $\begin{array}{c}0.09 \\
(0.04)\end{array}$ & $\begin{array}{c}0.02 \\
(0.03)\end{array}$ & $\begin{array}{c}0.12^{* * *} \\
(0.03)\end{array}$ & $\begin{array}{l}-0.01 \\
(0.04)\end{array}$ & $\begin{array}{c}1.31 \\
(0.24)\end{array}$ & $\begin{array}{c}2.97^{* * *} \\
(0.51)\end{array}$ & $\begin{array}{c}0.59 \\
(0.15)\end{array}$ & $\begin{array}{l}1.45 \\
(0.25)\end{array}$ & $\begin{array}{c}1.17 \\
(0.28)\end{array}$ & $\begin{array}{c}2.33^{* * *} \\
(0.51)\end{array}$ & $\begin{array}{c}0.95 \\
(0.22)\end{array}$ \\
\hline Wave $=2$ & $\begin{array}{c}-0.32 * * * \\
(0.06)\end{array}$ & $\begin{array}{c}-0.20 * * * \\
(0.04)\end{array}$ & $\begin{array}{c}0.01 \\
(0.05)\end{array}$ & $\begin{array}{c}0.07 \\
(0.06)\end{array}$ & $\begin{array}{c}0.06 \\
(0.04)\end{array}$ & $\begin{array}{l}-0.02 \\
(0.03)\end{array}$ & $\begin{array}{c}0.38^{* * *} \\
(0.06)\end{array}$ & $\begin{array}{c}0.25^{* * *} \\
(0.07)\end{array}$ & $\begin{array}{c}0.27 * * * \\
(0.09)\end{array}$ & $\begin{array}{c}1.13 \\
(0.48)\end{array}$ & $\begin{array}{c}1.34 \\
(0.34)\end{array}$ & $\begin{array}{c}1.78 \\
(0.64)\end{array}$ & $\begin{array}{c}0.85 \\
(0.26)\end{array}$ & $\begin{array}{c}5.51^{* * *} \\
(1.53)\end{array}$ \\
\hline Secondary * Wave 2 & $\begin{array}{c}0.00 \\
(0.06)\end{array}$ & $\begin{array}{l}-0.08 \\
(0.05)\end{array}$ & $\begin{array}{c}0.04 \\
(0.05)\end{array}$ & $\begin{array}{l}-0.06 \\
(0.07)\end{array}$ & $\begin{array}{l}-0.08 \\
(0.05)\end{array}$ & $\begin{array}{l}0.04 \\
(0.04)\end{array}$ & $\begin{array}{l}-0.06 \\
(0.06)\end{array}$ & $\begin{array}{c}0.99 \\
(0.30)\end{array}$ & $\begin{array}{c}0.81 \\
(0.31)\end{array}$ & $\begin{array}{c}1.36 \\
(0.66)\end{array}$ & $\begin{array}{c}0.77 \\
(0.22)\end{array}$ & $\begin{array}{c}0.49 \\
(0.21)\end{array}$ & $\begin{array}{c}1.36 \\
(0.49)\end{array}$ & $\begin{array}{c}0.78 \\
(0.25)\end{array}$ \\
\hline $\begin{array}{l}\text { More than Secondary } \\
\text { * Wave } 2\end{array}$ & $\begin{array}{l}-0.00 \\
(0.06)\end{array}$ & $\begin{array}{l}-0.12 \\
(0.05)\end{array}$ & $\begin{array}{c}0.06 \\
(0.05)\end{array}$ & $\begin{array}{l}-0.13 \\
(0.07)\end{array}$ & $\begin{array}{l}-0.07 \\
(0.04)\end{array}$ & $\begin{array}{l}0.00 \\
(0.04)\end{array}$ & $\begin{array}{l}-0.07 \\
(0.06)\end{array}$ & $\begin{array}{c}0.93 \\
(0.27)\end{array}$ & $\begin{array}{c}0.90 \\
(0.33)\end{array}$ & $\begin{array}{c}1.56 \\
(0.70)\end{array}$ & $\begin{array}{c}0.58 \\
(0.17)\end{array}$ & $\begin{array}{c}0.50 \\
(0.20)\end{array}$ & $\begin{array}{c}1.08 \\
(0.36)\end{array}$ & $\begin{array}{l}0.78 \\
(0.24)\end{array}$ \\
\hline $\begin{array}{l}\text { Day Diary } \\
\text { Completed: } \\
\text { Omitted=Monday }\end{array}$ & & & & & & & & & & & & & & \\
\hline Tuesday & $\begin{array}{l}0.01 \\
(0.03)\end{array}$ & $\begin{array}{l}-0.01 \\
(0.03)\end{array}$ & $\begin{array}{l}0.02 \\
(0.02)\end{array}$ & $\begin{array}{c}-0.10 * * \\
(0.03)\end{array}$ & $\begin{array}{c}0.05 \\
(0.02)\end{array}$ & $\begin{array}{l}-0.01 \\
(0.02)\end{array}$ & $\begin{array}{l}-0.02 \\
(0.03)\end{array}$ & $\begin{array}{l}1.03 \\
(0.15)\end{array}$ & $\begin{array}{l}0.95 \\
(0.15)\end{array}$ & $\begin{array}{c}1.20 \\
(0.31)\end{array}$ & $\begin{array}{l}0.66^{* *} \\
(0.09)\end{array}$ & $\begin{array}{l}1.65 * \\
(0.34)\end{array}$ & $\begin{array}{l}0.91 \\
(0.15)\end{array}$ & $\begin{array}{l}0.91 \\
(0.14)\end{array}$ \\
\hline Wednesday & $\begin{array}{l}0.06 \\
(0.03)\end{array}$ & $\begin{array}{l}-0.00 \\
(0.03)\end{array}$ & $\begin{array}{l}-0.03 \\
(0.03)\end{array}$ & $\begin{array}{l}-0.03 \\
(0.04)\end{array}$ & $\begin{array}{l}-0.00 \\
(0.03)\end{array}$ & $\begin{array}{l}0.02 \\
(0.03)\end{array}$ & $\begin{array}{l}-0.04 \\
(0.03)\end{array}$ & $\begin{array}{c}1.31 \\
(0.20)\end{array}$ & $\begin{array}{l}0.99 \\
(0.16)\end{array}$ & $\begin{array}{c}0.75 \\
(0.20)\end{array}$ & $\begin{array}{c}0.89 \\
(0.13)\end{array}$ & $\begin{array}{c}0.98 \\
(0.22)\end{array}$ & $\begin{array}{c}1.11 \\
(0.19)\end{array}$ & $\begin{array}{c}0.81 \\
(0.13)\end{array}$ \\
\hline Thursday & 0.04 & -0.01 & 0.01 & -0.03 & 0.02 & 0.01 & -0.02 & 1.23 & 0.94 & 1.15 & 0.87 & 1.18 & 1.04 & 0.91 \\
\hline
\end{tabular}




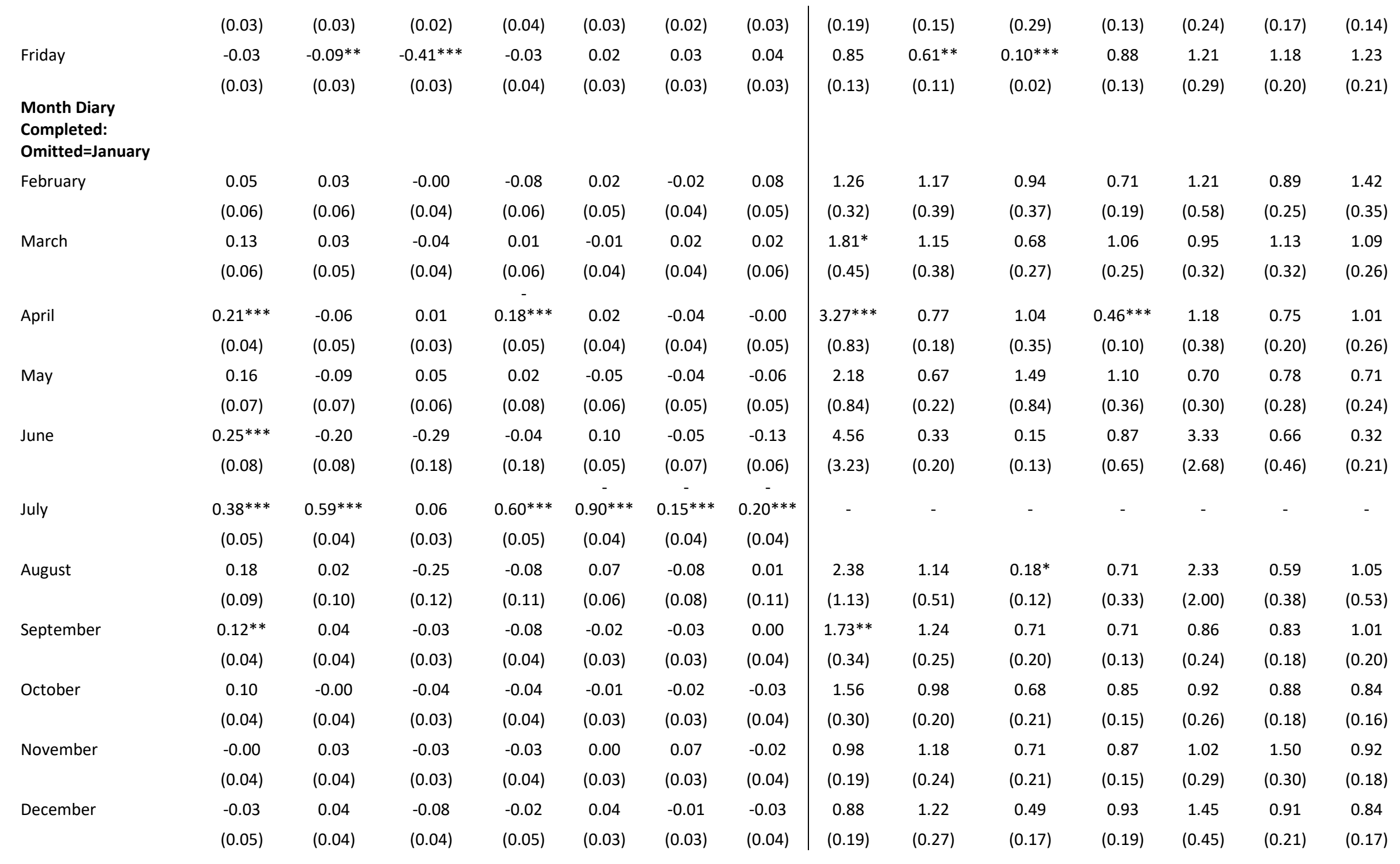




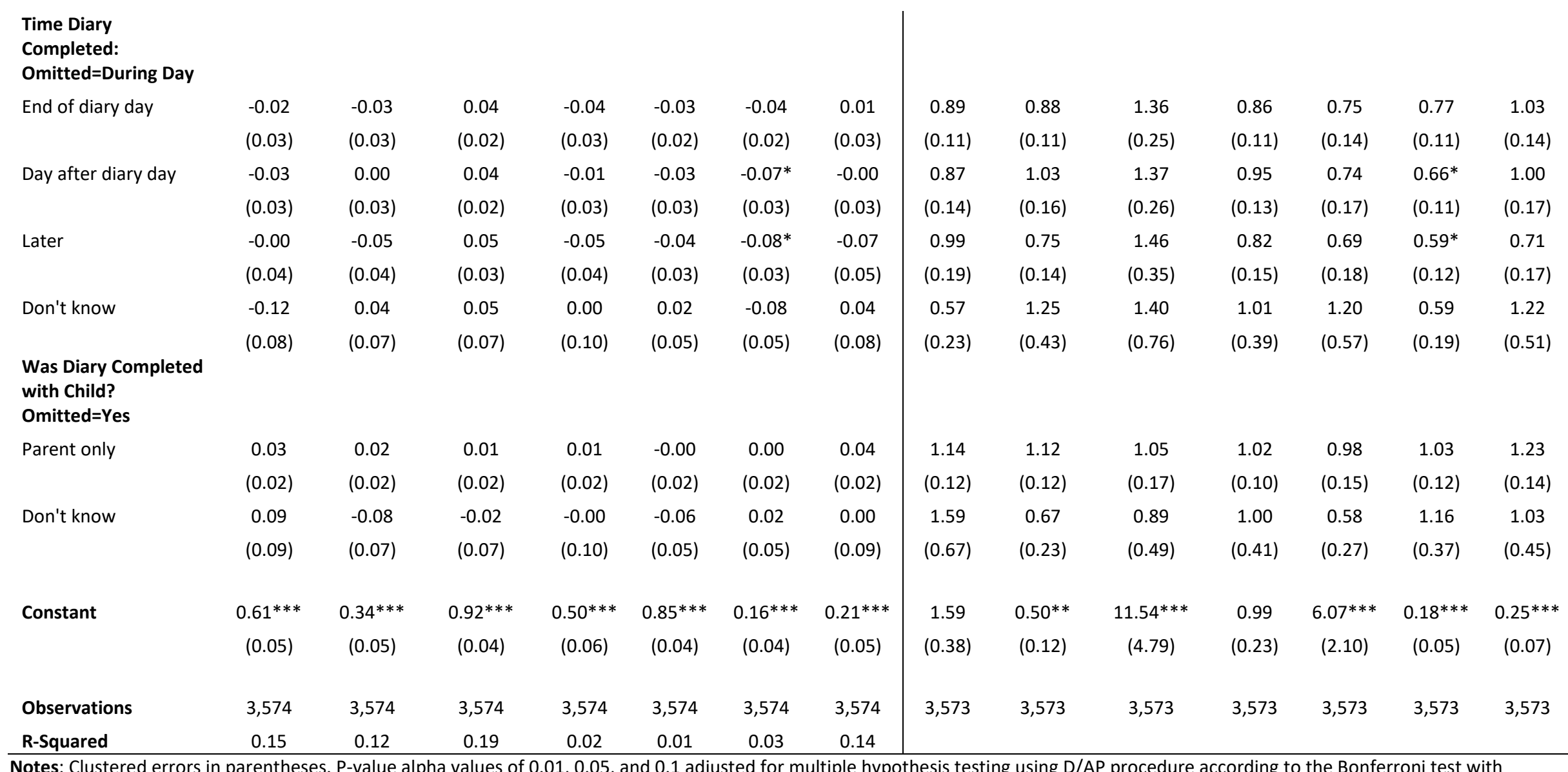

Notes: Clustered errors in parentheses. P-value alpha values of $0.01,0.05$, and 0.1 adjusted for multiple hypothesis testing using D/AP procedure according to the Bonferroni test with correlation $=0.15$ resulting in $p$-value cut-offs: ${ }^{* * *} p<0.0016,{ }^{* *} p<0.008,{ }^{*} p<0.016$ 
Table A8 Full Table for Girls' Time

\begin{tabular}{|c|c|c|c|c|c|c|c|c|c|c|c|c|c|c|}
\hline \multirow[b]{2}{*}{ Variables } & \multicolumn{7}{|c|}{ Linear regression } & \multicolumn{7}{|c|}{ Negative binomial } \\
\hline & $\begin{array}{l}\text { Sport } \\
\text { (Min) }\end{array}$ & $\begin{array}{l}\text { Reading } \\
\text { (Min) }\end{array}$ & $\begin{array}{l}\text { Homework } \\
\text { (Min) }\end{array}$ & $\begin{array}{c}\text { Playing } \\
\text { (Min) }\end{array}$ & $\begin{array}{l}\text { Media } \\
\text { (Min) }\end{array}$ & $\begin{array}{l}\text { Hobbies } \\
\text { (Min) }\end{array}$ & $\begin{array}{l}\text { Family } \\
\text { (Min) }\end{array}$ & $\begin{array}{l}\text { Sport } \\
\text { (IRR) }\end{array}$ & $\begin{array}{l}\text { Reading } \\
\text { (IRR) }\end{array}$ & $\begin{array}{l}\text { Homework } \\
\text { (IRR) }\end{array}$ & $\begin{array}{c}\text { Playing } \\
\text { (IRR) }\end{array}$ & $\begin{array}{l}\text { Media } \\
\text { (IRR) }\end{array}$ & $\begin{array}{l}\text { Hobbies } \\
\text { (IRR) }\end{array}$ & $\begin{array}{l}\text { Family } \\
\text { (IRR) }\end{array}$ \\
\hline \multicolumn{15}{|l|}{$\begin{array}{l}\text { Mother's Education: } \\
\text { Omitted=Less than Secondary }\end{array}$} \\
\hline \multirow[t]{2}{*}{ Secondary } & -5.28 & 3.97 & -0.16 & 0.95 & 2.77 & 3.52 & 3.66 & 0.91 & 1.29 & 0.99 & 1.07 & 1.05 & 1.32 & 1.50 \\
\hline & $(4.38)$ & $(2.17)$ & $(2.54)$ & $(4.36)$ & $(4.21)$ & $(2.67)$ & $(2.87)$ & $(0.08)$ & $(0.16)$ & $(0.06)$ & $(0.11)$ & $(0.06)$ & $(0.23)$ & $(0.29)$ \\
\hline \multirow[t]{2}{*}{ More than Secondary } & -8.37 & $6.82 * * *$ & -3.39 & -0.25 & -2.08 & $7.87 * * *$ & 1.61 & 0.86 & $1.45^{* * *}$ & 0.91 & 1.04 & 0.98 & $1.74 * * *$ & 1.30 \\
\hline & $(4.25)$ & (1.95) & $(2.36)$ & $(4.13)$ & $(4.01)$ & $(2.44)$ & $(2.96)$ & $(0.08)$ & $(0.16)$ & $(0.05)$ & $(0.11)$ & $(0.06)$ & $(0.27)$ & $(0.24)$ \\
\hline \multirow[t]{2}{*}{ Wave $=2$} & $-40.80 * * *$ & $-9.02 * *$ & $31.88 * * *$ & 14.15 & $41.68^{* * *}$ & 6.91 & $28.56^{* * *}$ & $0.20 * * *$ & $0.39 * * *$ & $1.80 * * *$ & 1.34 & $1.63 * * *$ & 1.55 & $3.72^{* * *}$ \\
\hline & $(4.13)$ & $(2.93)$ & $(6.81)$ & (7.35) & $(11.00)$ & $(5.35)$ & (6.19) & $(0.04)$ & $(0.12)$ & $(0.18)$ & $(0.19)$ & $(0.17)$ & $(0.44)$ & $(0.81)$ \\
\hline \multirow[t]{2}{*}{ Secondary * Wave 2} & $14.15^{* *}$ & -2.17 & 2.36 & -16.98 & -12.90 & -9.06 & 4.73 & $2.01^{* *}$ & 1.04 & 0.96 & $0.66^{*}$ & 0.86 & $0.50 * *$ & 0.83 \\
\hline & $(5.37)$ & (3.23) & (7.25) & $(8.31)$ & (11.98) & $(5.48)$ & $(7.04)$ & $(0.46)$ & $(0.34)$ & $(0.11)$ & $(0.11)$ & $(0.10)$ & $(0.16)$ & $(0.21)$ \\
\hline \multirow[t]{2}{*}{ More than Secondary * Wave 2} & $25.38^{* * *}$ & 0.09 & 3.36 & $-20.26 *$ & -20.13 & -9.09 & -0.73 & $3.06 * * *$ & 1.43 & 1.02 & $0.59 * *$ & 0.81 & 0.59 & 0.79 \\
\hline & $(5.15)$ & (3.36) & (7.19) & $(8.03)$ & $(11.56)$ & (5.29) & $(6.84)$ & $(0.65)$ & $(0.45)$ & $(0.11)$ & $(0.10)$ & $(0.09)$ & $(0.18)$ & $(0.20)$ \\
\hline \multirow{2}{*}{\multicolumn{15}{|c|}{$\begin{array}{l}\text { Day Diary Completed: } \\
\text { Omitted=Monday }\end{array}$}} \\
\hline & & & & & & & & & & & & & & \\
\hline \multirow[t]{2}{*}{ Tuesday } & $7.26^{*}$ & -0.71 & 0.26 & 0.65 & -4.71 & -1.04 & -4.17 & 1.24 & 0.92 & 1.01 & 1.03 & 0.94 & 0.98 & 0.92 \\
\hline & $(2.88)$ & $(1.51)$ & $(2.60)$ & $(3.63)$ & $(4.54)$ & $(2.65)$ & (3.13) & $(0.13)$ & $(0.11)$ & $(0.04)$ & $(0.10)$ & $(0.05)$ & $(0.14)$ & $(0.12)$ \\
\hline \multirow[t]{2}{*}{ Wednesday } & $6.33^{*}$ & 2.30 & 0.67 & 0.39 & 4.63 & 0.43 & $-8.23 * *$ & 1.19 & 1.11 & 1.00 & 1.01 & 1.04 & 1.15 & $0.65 * * *$ \\
\hline & (3.37) & (1.94) & $(2.76)$ & $(3.81)$ & $(5.85)$ & $(2.70)$ & (3.16) & $(0.14)$ & $(0.15)$ & $(0.04)$ & $(0.11)$ & $(0.06)$ & $(0.16)$ & $(0.08)$ \\
\hline \multirow[t]{2}{*}{ Thursday } & 4.67 & -1.48 & 2.86 & 4.18 & -2.88 & -4.33 & -3.79 & 1.06 & 0.79 & 1.03 & 1.11 & 0.96 & 0.79 & 0.92 \\
\hline & (3.03) & $(1.70)$ & (3.11) & (3.54) & $(5.00)$ & (2.56) & (3.47) & $(0.12)$ & $(0.10)$ & $(0.04)$ & $(0.10)$ & $(0.05)$ & $(0.12)$ & $(0.12)$ \\
\hline
\end{tabular}




\begin{tabular}{|c|c|c|c|c|c|c|c|c|c|c|c|c|c|c|}
\hline Friday & $\begin{array}{c}10.03 * * \\
(3.39)\end{array}$ & $\begin{array}{c}1.05 \\
(2.29)\end{array}$ & $\begin{array}{c}-25.82 * * * \\
(3.72)\end{array}$ & $\begin{array}{c}20.62 * * * \\
(5.12)\end{array}$ & $\begin{array}{l}12.34 \\
(5.57)\end{array}$ & $\begin{array}{l}-1.66 \\
(2.82)\end{array}$ & $\begin{array}{c}14.41 * * * \\
(4.44)\end{array}$ & $\begin{array}{c}1.27 \\
(0.15)\end{array}$ & $\begin{array}{c}1.07 \\
(0.18)\end{array}$ & $\begin{array}{c}0.54 * * * \\
(0.04)\end{array}$ & $\begin{array}{c}1.58^{* * *} \\
(0.17)\end{array}$ & $\begin{array}{l}1.18^{* *} \\
(0.07)\end{array}$ & $\begin{array}{c}0.91 \\
(0.15)\end{array}$ & $\begin{array}{c}1.84 * * * \\
(0.26)\end{array}$ \\
\hline \multicolumn{15}{|c|}{$\begin{array}{l}\text { Month Diary Completed: } \\
\text { Omitted=January }\end{array}$} \\
\hline February & $\begin{array}{c}8.19 \\
(4.53)\end{array}$ & $\begin{array}{l}-8.90 \\
(4.22)\end{array}$ & $\begin{array}{l}-1.44 \\
(5.66)\end{array}$ & $\begin{array}{l}-8.19 \\
(7.51)\end{array}$ & $\begin{array}{l}-3.38 \\
(8.68)\end{array}$ & $\begin{array}{c}6.97 \\
(6.50)\end{array}$ & $\begin{array}{l}-3.26 \\
(6.59)\end{array}$ & $\begin{array}{l}1.43 \\
(0.28)\end{array}$ & $\begin{array}{l}0.45^{* *} \\
(0.12)\end{array}$ & $\begin{array}{l}1.07 \\
(0.10)\end{array}$ & $\begin{array}{c}0.82 \\
(0.15)\end{array}$ & $\begin{array}{c}0.96 \\
(0.09)\end{array}$ & $\begin{array}{c}1.50 \\
(0.48)\end{array}$ & $\begin{array}{l}1.25 \\
(0.34)\end{array}$ \\
\hline March & $\begin{array}{c}18.29 * * * \\
(5.11)\end{array}$ & $\begin{array}{l}-5.58 \\
(4.40)\end{array}$ & $\begin{array}{l}-7.78 \\
(6.09)\end{array}$ & $\begin{array}{l}-5.19 \\
(7.79)\end{array}$ & $\begin{array}{c}1.14 \\
(11.48)\end{array}$ & $\begin{array}{l}-6.35 \\
(3.64)\end{array}$ & $\begin{array}{l}-3.47 \\
(6.45)\end{array}$ & $\begin{array}{c}2.29 * * * \\
(0.49)\end{array}$ & $\begin{array}{c}0.61 \\
(0.18)\end{array}$ & $\begin{array}{c}0.96 \\
(0.08)\end{array}$ & $\begin{array}{c}0.83 \\
(0.15)\end{array}$ & $\begin{array}{c}0.97 \\
(0.11)\end{array}$ & $\begin{array}{c}0.56 \\
(0.16)\end{array}$ & $\begin{array}{c}1.06 \\
(0.22)\end{array}$ \\
\hline April & $\begin{array}{c}24.97 * * * \\
(6.02)\end{array}$ & $\begin{array}{l}-5.58 \\
(4.06)\end{array}$ & $\begin{array}{l}-2.54 \\
(4.21)\end{array}$ & $\begin{array}{l}-8.63 \\
(7.16)\end{array}$ & $\begin{array}{l}-8.72 \\
(7.56)\end{array}$ & $\begin{array}{l}-1.93 \\
(4.16)\end{array}$ & $\begin{array}{c}0.57 \\
(4.92)\end{array}$ & $\begin{array}{c}2.03 * * * \\
(0.32)\end{array}$ & $\begin{array}{c}0.61 \\
(0.14)\end{array}$ & $\begin{array}{c}1.02 \\
(0.08)\end{array}$ & $\begin{array}{c}0.79 \\
(0.14)\end{array}$ & $\begin{array}{c}0.90 \\
(0.08)\end{array}$ & $\begin{array}{c}0.81 \\
(0.21)\end{array}$ & $\begin{array}{c}1.07 \\
(0.28)\end{array}$ \\
\hline May & $\begin{array}{c}34.29 * * \\
(11.75)\end{array}$ & $\begin{array}{l}-8.12 \\
(4.94)\end{array}$ & $\begin{array}{c}2.99 \\
(7.96)\end{array}$ & $\begin{array}{l}-18.19 \\
(8.00)\end{array}$ & $\begin{array}{l}-15.61 \\
(10.53)\end{array}$ & $\begin{array}{c}5.40 \\
(8.06)\end{array}$ & $\begin{array}{l}-0.89 \\
(5.76)\end{array}$ & $\begin{array}{c}2.30^{* * *} \\
(0.53)\end{array}$ & $\begin{array}{c}0.49 \\
(0.16)\end{array}$ & $\begin{array}{c}1.08 \\
(0.13)\end{array}$ & $\begin{array}{c}0.61 \\
(0.14)\end{array}$ & $\begin{array}{c}0.84 \\
(0.11)\end{array}$ & $\begin{array}{c}1.22 \\
(0.41)\end{array}$ & $\begin{array}{c}0.99 \\
(0.29)\end{array}$ \\
\hline June & $\begin{array}{c}52.91^{* * *} \\
(12.20)\end{array}$ & $\begin{array}{l}-0.42 \\
(5.97)\end{array}$ & $\begin{array}{l}-9.51 \\
(6.80)\end{array}$ & $\begin{array}{c}-3.54 \\
(10.24)\end{array}$ & $\begin{array}{c}-9.52 \\
(13.08)\end{array}$ & $\begin{array}{l}-6.65 \\
(4.74)\end{array}$ & $\begin{array}{l}-2.26 \\
(9.44)\end{array}$ & $\begin{array}{c}3.22^{* * *} \\
(0.62)\end{array}$ & $\begin{array}{c}0.84 \\
(0.25)\end{array}$ & $\begin{array}{c}0.92 \\
(0.14)\end{array}$ & $\begin{array}{c}0.90 \\
(0.19)\end{array}$ & $\begin{array}{c}0.95 \\
(0.16)\end{array}$ & $\begin{array}{c}0.54 \\
(0.22)\end{array}$ & $\begin{array}{c}1.13 \\
(0.60)\end{array}$ \\
\hline July & & & & & & & & & & & & & & \\
\hline August & $\begin{array}{c}28.60 \\
(18.65)\end{array}$ & $\begin{array}{l}-3.47 \\
(8.62)\end{array}$ & $\begin{array}{l}11.10 \\
(9.58)\end{array}$ & $\begin{array}{l}-17.80 \\
(12.84)\end{array}$ & $\begin{array}{c}6.21 \\
(28.89)\end{array}$ & $\begin{array}{c}-12.26 * * \\
(4.20)\end{array}$ & $\begin{array}{c}14.35 \\
(18.54)\end{array}$ & $\begin{array}{c}1.96 \\
(0.62)\end{array}$ & $\begin{array}{c}0.62 \\
(0.31)\end{array}$ & $\begin{array}{c}1.33 \\
(0.30)\end{array}$ & $\begin{array}{c}0.74 \\
(0.23)\end{array}$ & $\begin{array}{c}1.07 \\
(0.32)\end{array}$ & $\begin{array}{c}0.13^{* *} \\
(0.09)\end{array}$ & $\begin{array}{c}1.90 \\
(1.05)\end{array}$ \\
\hline September & $\begin{array}{c}16.11^{* * *} \\
(4.36)\end{array}$ & $\begin{array}{l}-7.10^{*} \\
(4.00)\end{array}$ & $\begin{array}{c}2.78 \\
(5.09)\end{array}$ & $\begin{array}{l}-3.28 \\
(6.75)\end{array}$ & $\begin{array}{l}-7.74 \\
(8.19)\end{array}$ & $\begin{array}{l}-2.75 \\
(3.87)\end{array}$ & $\begin{array}{l}-2.68 \\
(5.56)\end{array}$ & $\begin{array}{c}1.76^{* * *} \\
(0.29)\end{array}$ & $\begin{array}{c}0.54^{* *} \\
(0.13)\end{array}$ & $\begin{array}{c}1.13 \\
(0.09)\end{array}$ & $\begin{array}{c}0.89 \\
(0.13)\end{array}$ & $\begin{array}{c}0.90 \\
(0.07)\end{array}$ & $\begin{array}{c}0.76 \\
(0.17)\end{array}$ & $\begin{array}{c}0.99 \\
(0.21)\end{array}$ \\
\hline October & $\begin{array}{c}16.72^{* * *} \\
(3.96)\end{array}$ & $\begin{array}{l}-4.67 \\
(4.09)\end{array}$ & $\begin{array}{l}-4.58 \\
(4.38)\end{array}$ & $\begin{array}{l}-7.61 \\
(6.14)\end{array}$ & $\begin{array}{l}-13.68 \\
(7.23)\end{array}$ & $\begin{array}{c}0.40 \\
(3.90)\end{array}$ & $\begin{array}{c}3.26 \\
(4.75)\end{array}$ & $\begin{array}{c}1.74^{* * *} \\
(0.26)\end{array}$ & $\begin{array}{c}0.66 \\
(0.15)\end{array}$ & $\begin{array}{c}1.00 \\
(0.06)\end{array}$ & $\begin{array}{c}0.80 \\
(0.11)\end{array}$ & $\begin{array}{c}0.83^{* *} \\
(0.07)\end{array}$ & $\begin{array}{c}1.02 \\
(0.23)\end{array}$ & $\begin{array}{c}1.25 \\
(0.24)\end{array}$ \\
\hline November & $\begin{array}{c}4.06 \\
(3.85)\end{array}$ & $\begin{array}{l}-5.30 \\
(3.99)\end{array}$ & $\begin{array}{l}-0.09 \\
(4.30)\end{array}$ & $\begin{array}{r}-10.01 \\
(6.16)\end{array}$ & $\begin{array}{l}-12.92 \\
(7.50)\end{array}$ & $\begin{array}{c}2.20 \\
(3.70)\end{array}$ & $\begin{array}{c}7.72 \\
(5.12)\end{array}$ & $\begin{array}{c}1.26 \\
(0.20)\end{array}$ & $\begin{array}{c}0.65 \\
(0.16)\end{array}$ & $\begin{array}{c}1.05 \\
(0.06)\end{array}$ & $\begin{array}{c}0.73 \\
(0.10)\end{array}$ & $\begin{array}{c}0.87 \\
(0.07)\end{array}$ & $\begin{array}{c}1.12 \\
(0.24)\end{array}$ & $\begin{array}{c}1.44 \\
(0.29)\end{array}$ \\
\hline December & $\begin{array}{c}0.00 \\
(3.99)\end{array}$ & $\begin{array}{l}-8.52 \\
(4.16)\end{array}$ & $\begin{array}{l}-9.16 \\
(5.11)\end{array}$ & $\begin{array}{l}-3.65 \\
(6.40)\end{array}$ & $\begin{array}{c}0.14 \\
(8.67)\end{array}$ & $\begin{array}{c}1.37 \\
(4.33)\end{array}$ & $\begin{array}{c}7.79 \\
(5.48)\end{array}$ & $\begin{array}{c}1.07 \\
(0.19)\end{array}$ & $\begin{array}{c}0.51^{* *} \\
(0.13)\end{array}$ & $\begin{array}{c}0.91 \\
(0.07)\end{array}$ & $\begin{array}{c}0.92 \\
(0.13)\end{array}$ & $\begin{array}{c}1.02 \\
(0.09)\end{array}$ & $\begin{array}{c}1.08 \\
(0.27)\end{array}$ & $\begin{array}{c}1.32 \\
(0.27)\end{array}$ \\
\hline
\end{tabular}


Time Diary Completed:

Omitted=During Day

End of diary day

Day after diary day

$\begin{array}{cccc}-3.83 & -1.09 & 3.38 & -0.43 \\ (2.87) & (1.54) & (2.57) & (3.25)\end{array}$

$\begin{array}{llll}4.53 & -6.12 & -2.99 & -3.00\end{array}$

$\begin{array}{lllllll}-4.39 & 2.93 & -3.34 & 5.90 & 5.75 & -3.87 & -0.60\end{array}$

Later

Don't know

$(3.89) \quad(3.31) \quad(3.83) \quad(6.00) \quad(6.83) \quad(2.79)$

$\begin{array}{lllllll}10.26 & -1.72 & 20.46 * * * & -0.64 & -10.41 & -2.45 & -5.68\end{array}$

(4.56)

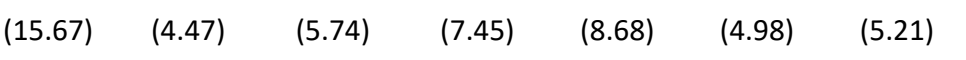

$\begin{array}{ccccccc}0.85 & 0.88 & 1.09 & 1.00 & 0.94 & 0.83 & 1.02 \\ (0.08) & (0.10) & (0.05) & (0.08) & (0.04) & (0.10) & (0.12) \\ 1.08 & 1.05 & 1.10 & 1.13 & 0.93 & 0.90 & 0.87 \\ (0.11) & (0.13) & (0.06) & (0.12) & (0.06) & (0.13) & (0.12) \\ 0.80 & 1.12 & 0.98 & 1.13 & 1.04 & 0.81 & 1.19 \\ (0.10) & (0.19) & (0.06) & (0.14) & (0.07) & (0.13) & (0.24) \\ 1.01 & 0.87 & 1.58^{* * *} & 0.99 & 0.91 & 1.00 & 0.38 \\ (0.21) & (0.25) & (0.21) & (0.21) & (0.09) & (0.40) & (0.19)\end{array}$

Was Diary Completed with

Child? Omitted=Yes

Parent only

$\begin{array}{llllll}3.77 & -1.69 & 0.56 & -1.26 & -11.69 * * * & -3.59\end{array}$

$\begin{array}{llllll}(2.26) & (1.26) \quad(2.37) \quad(2.86) \quad(3.39) \quad \text { (1.62) }\end{array}$

Don't know

$-10.05$

(14.58)

$\begin{array}{lllll}2.77 & -19.73^{* * *} & 3.11 & -2.59 & -0.38\end{array}$

4.31

1.14

D.

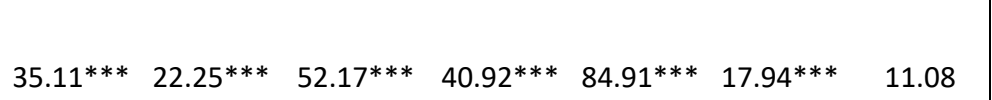

(0.20)

$1.31 \quad 0.66^{* * *} \quad 1.10$

$0.94 \quad 0.84 \quad 2.99$

Constant

$\begin{array}{lcccccccccccccc} & (5.51) & (3.90) & (4.45) & (7.47) & (8.50) & (4.68) & (5.23) & (5.21) & (6.45) & (3.68) & (6.55) & (7.69) & (4.35) & (2.49) \\ \text { Observations } & 3,606 & 3,606 & 3,606 & 3,606 & 3,606 & 3,606 & 3,606 & 3,606 & 3,606 & 3,606 & 3,606 & 3,606 & 3,606 & 3,606 \\ \text { R-Squared } & 0.14 & 0.05 & 0.17 & 0.04 & 0.07 & 0.02 & 0.11 & & & \end{array}$

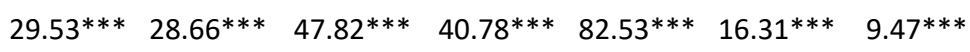

Notes: Clustered errors in parentheses. P-value alpha values of $0.01,0.05$, and 0.1 adjusted for multiple hypothesis testing using D/AP procedure according to the Bonferroni test with correlation $=0.15$ resulting in $p$-value cut-offs: ${ }^{* * *} p<0.0019,{ }^{* *} p<0.0096,{ }^{*} p<0.02$ 
Table A9 Full Table for Boys' Time

\begin{tabular}{|c|c|c|c|c|c|c|c|c|c|c|c|c|c|c|}
\hline \multirow[b]{2}{*}{ Variables } & \multicolumn{7}{|c|}{ Linear regression } & \multicolumn{7}{|c|}{ Negative binomial } \\
\hline & $\begin{array}{l}\text { Sport } \\
\text { (Min) }\end{array}$ & $\begin{array}{l}\text { Reading } \\
\text { (Min) }\end{array}$ & $\begin{array}{l}\text { Homework } \\
\text { (Min) }\end{array}$ & $\begin{array}{l}\text { Playing } \\
\text { (Min) }\end{array}$ & $\begin{array}{l}\text { Media } \\
\text { (Min) }\end{array}$ & $\begin{array}{l}\text { Hobbies } \\
\text { (Min) }\end{array}$ & $\begin{array}{l}\text { Family } \\
\text { (Min) }\end{array}$ & $\begin{array}{l}\text { Sport } \\
\text { (IRR) }\end{array}$ & $\begin{array}{l}\text { Reading } \\
\text { (IRR) }\end{array}$ & $\begin{array}{l}\text { Homework } \\
\text { (IRR) }\end{array}$ & $\begin{array}{l}\text { Playing } \\
\text { (IRR) }\end{array}$ & $\begin{array}{l}\text { Media } \\
\text { (IRR) }\end{array}$ & $\begin{array}{l}\text { Hobbies } \\
\text { (IRR) }\end{array}$ & $\begin{array}{l}\text { Family } \\
\text { (IRR) }\end{array}$ \\
\hline \multicolumn{15}{|c|}{$\begin{array}{l}\text { Mother's Education: } \\
\text { Omitted=Less than Secondary }\end{array}$} \\
\hline Secondary & $\begin{array}{l}-1.67 \\
(5.54)\end{array}$ & $\begin{array}{c}4.09 \\
(1.87)\end{array}$ & $\begin{array}{l}-4.09 \\
(2.66)\end{array}$ & $\begin{array}{c}2.34 \\
(3.86)\end{array}$ & $\begin{array}{l}-2.21 \\
(4.95)\end{array}$ & $\begin{array}{c}0.77 \\
(2.05)\end{array}$ & $\begin{array}{l}-1.92 \\
(4.96)\end{array}$ & $\begin{array}{c}1.02 \\
(0.08)\end{array}$ & $\begin{array}{c}1.46 * * \\
(0.21)\end{array}$ & $\begin{array}{c}0.88 \\
(0.05)\end{array}$ & $\begin{array}{c}1.05 \\
(0.14)\end{array}$ & $\begin{array}{l}0.97 \\
(0.06)\end{array}$ & $\begin{array}{c}1.24 \\
(0.28)\end{array}$ & $\begin{array}{c}0.93 \\
(0.23)\end{array}$ \\
\hline More than Secondary & $\begin{array}{l}-3.42 \\
(5.10)\end{array}$ & $\begin{array}{c}11.40 * * * \\
(2.04)\end{array}$ & $\begin{array}{c}-6.94 * * \\
(2.45)\end{array}$ & $\begin{array}{c}3.34 \\
(3.52)\end{array}$ & $\begin{array}{l}-4.22 \\
(4.84)\end{array}$ & $\begin{array}{c}4.47 \\
(2.00)\end{array}$ & $\begin{array}{l}-4.04 \\
(4.77)\end{array}$ & $\begin{array}{c}0.98 \\
(0.07)\end{array}$ & $\begin{array}{c}2.10^{* * *} \\
(0.28)\end{array}$ & $\begin{array}{c}0.80 * * * \\
(0.05)\end{array}$ & $\begin{array}{c}1.16 \\
(0.15)\end{array}$ & $\begin{array}{c}0.94 \\
(0.06)\end{array}$ & $\begin{array}{l}1.91 * * \\
(0.41)\end{array}$ & $\begin{array}{c}0.77 \\
(0.18)\end{array}$ \\
\hline Wave $=2$ & $\begin{array}{c}-38.76^{* * *} \\
(6.44)\end{array}$ & $\begin{array}{c}-7.65 * * * \\
(2.16)\end{array}$ & $\begin{array}{c}15.52^{* *} \\
(5.50)\end{array}$ & $\begin{array}{l}19.27 \\
(8.38)\end{array}$ & $\begin{array}{c}38.16^{* * *} \\
(8.52)\end{array}$ & $\begin{array}{l}-1.12 \\
(2.60)\end{array}$ & $\begin{array}{c}37.41 * * * \\
(8.62)\end{array}$ & $\begin{array}{c}0.45^{* * *} \\
(0.09)\end{array}$ & $\begin{array}{c}0.39 * * \\
(0.12)\end{array}$ & $\begin{array}{c}1.39 * * \\
(0.15)\end{array}$ & $\begin{array}{c}1.72^{* *} \\
(0.34)\end{array}$ & $\begin{array}{c}1.46^{* * *} \\
(0.12)\end{array}$ & $\begin{array}{c}0.94 \\
(0.30)\end{array}$ & $\begin{array}{c}3.29 * * * \\
(0.84)\end{array}$ \\
\hline Secondary * Wave 2 & $\begin{array}{c}20.00 \\
(11.44)\end{array}$ & $\begin{array}{l}-2.81 \\
(2.67)\end{array}$ & $\begin{array}{l}13.35 \\
(6.27)\end{array}$ & $\begin{array}{l}-8.21 \\
(9.51)\end{array}$ & $\begin{array}{l}-10.53 \\
(9.99)\end{array}$ & $\begin{array}{c}5.89 \\
(3.49)\end{array}$ & $\begin{array}{l}-8.96 \\
(9.54)\end{array}$ & $\begin{array}{c}1.47 \\
(0.34)\end{array}$ & $\begin{array}{c}0.95 \\
(0.33)\end{array}$ & $\begin{array}{c}1.23 \\
(0.15)\end{array}$ & $\begin{array}{c}0.82 \\
(0.19)\end{array}$ & $\begin{array}{c}0.91 \\
(0.09)\end{array}$ & $\begin{array}{c}1.65 \\
(0.61)\end{array}$ & $\begin{array}{c}0.89 \\
(0.25)\end{array}$ \\
\hline More than Secondary & & & & & & & & & & & & & & \\
\hline 2 & $\begin{array}{l}11.71 \\
(7.04)\end{array}$ & $\begin{array}{l}-5.19 \\
(2.79)\end{array}$ & $\begin{array}{c}16.10^{* *} \\
(5.96)\end{array}$ & $\begin{array}{r}-18.50 \\
(8.78)\end{array}$ & $\begin{array}{l}-11.92 \\
(9.56)\end{array}$ & $\begin{array}{c}2.37 \\
(3.00)\end{array}$ & $\begin{array}{l}-9.45 \\
(9.14)\end{array}$ & $\begin{array}{c}1.23 \\
(0.24)\end{array}$ & $\begin{array}{c}1.16 \\
(0.37)\end{array}$ & $\begin{array}{l}1.33^{*} \\
(0.15)\end{array}$ & $\begin{array}{l}0.58^{*} \\
(0.13)\end{array}$ & $\begin{array}{c}0.90 \\
(0.08)\end{array}$ & $\begin{array}{c}1.10 \\
(0.37)\end{array}$ & $\begin{array}{c}1.03 \\
(0.28)\end{array}$ \\
\hline $\begin{array}{l}\text { Day Diary Completed: } \\
\text { Omitted=Monday }\end{array}$ & & & & & & & & & & & & & & \\
\hline Tuesday & $\begin{array}{c}1.78 \\
(3.98)\end{array}$ & $\begin{array}{l}-0.78 \\
(1.52)\end{array}$ & $\begin{array}{l}-1.19 \\
(2.63)\end{array}$ & $\begin{array}{l}-5.66 \\
(3.60)\end{array}$ & $\begin{array}{c}5.05 \\
(4.59)\end{array}$ & $\begin{array}{l}-0.95 \\
(1.85)\end{array}$ & $\begin{array}{l}-4.25 \\
(3.87)\end{array}$ & $\begin{array}{c}1.05 \\
(0.09)\end{array}$ & $\begin{array}{c}0.91 \\
(0.13)\end{array}$ & $\begin{array}{l}1.01 \\
(0.04)\end{array}$ & $\begin{array}{c}0.84 \\
(0.10)\end{array}$ & $\begin{array}{c}1.05 \\
(0.05)\end{array}$ & $\begin{array}{c}0.89 \\
(0.16)\end{array}$ & $\begin{array}{c}0.87 \\
(0.14)\end{array}$ \\
\hline Wednesday & $\begin{array}{c}9.33 \\
(8.19)\end{array}$ & $\begin{array}{c}0.50 \\
(1.83)\end{array}$ & $\begin{array}{r}-2.44 \\
(3.02)\end{array}$ & $\begin{array}{c}1.83 \\
(4.21)\end{array}$ & $\begin{array}{c}7.28 \\
(5.40)\end{array}$ & $\begin{array}{c}0.23 \\
(2.01)\end{array}$ & $\begin{array}{r}-2.66 \\
(4.17)\end{array}$ & $\begin{array}{c}1.19 \\
(0.14)\end{array}$ & $\begin{array}{c}1.01 \\
(0.15)\end{array}$ & $\begin{array}{c}0.97 \\
(0.05)\end{array}$ & $\begin{array}{c}1.02 \\
(0.12)\end{array}$ & $\begin{array}{c}1.06 \\
(0.06)\end{array}$ & $\begin{array}{c}1.14 \\
(0.21)\end{array}$ & $\begin{array}{c}0.90 \\
(0.13)\end{array}$ \\
\hline Thursday & 3.70 & -1.07 & 0.61 & -0.45 & 0.51 & -0.29 & -2.50 & 1.11 & 0.87 & 1.02 & 0.93 & 0.99 & 1.03 & 0.86 \\
\hline
\end{tabular}




\begin{tabular}{|c|c|c|c|c|c|c|c|c|c|c|c|c|c|c|}
\hline & $(4.24)$ & $(1.54)$ & $(2.75)$ & $(4.05)$ & $(4.63)$ & (1.85) & (3.86) & (0.10) & $(0.13)$ & $(0.04)$ & $(0.11)$ & $(0.05)$ & $(0.18)$ & (0.13) \\
\hline Friday & $\begin{array}{c}6.02 \\
(4.64)\end{array}$ & $\begin{array}{l}-2.65 \\
(1.76)\end{array}$ & $\begin{array}{c}-25.81^{* * *} \\
(3.23)\end{array}$ & $\begin{array}{c}9.58 \\
(4.71)\end{array}$ & $\begin{array}{c}22.08 * * * \\
(5.81)\end{array}$ & $\begin{array}{c}2.94 \\
(2.29)\end{array}$ & $\begin{array}{c}16.14 * * \\
(5.83)\end{array}$ & $\begin{array}{c}1.03 \\
(0.10)\end{array}$ & $\begin{array}{c}0.75 \\
(0.13)\end{array}$ & $\begin{array}{c}0.54 * * * \\
(0.04)\end{array}$ & $\begin{array}{c}1.26 \\
(0.14)\end{array}$ & $\begin{array}{c}1.24^{* * *} \\
(0.07)\end{array}$ & $\begin{array}{c}1.30 \\
(0.24)\end{array}$ & $\begin{array}{c}1.73^{* * *} \\
(0.27)\end{array}$ \\
\hline $\begin{array}{l}\text { Month Diary } \\
\text { Omitted=Jar }\end{array}$ & & & & & & & & & & & & & & \\
\hline February & $\begin{array}{c}8.82 \\
(6.67)\end{array}$ & $\begin{array}{c}0.47 \\
(4.03)\end{array}$ & $\begin{array}{l}-3.95 \\
(5.02)\end{array}$ & $\begin{array}{l}-8.99 \\
(7.66)\end{array}$ & $\begin{array}{c}2.33 \\
(9.10)\end{array}$ & $\begin{array}{l}-0.47 \\
(3.10)\end{array}$ & $\begin{array}{c}5.09 \\
(7.47)\end{array}$ & $\begin{array}{c}1.23 \\
(0.21)\end{array}$ & $\begin{array}{c}1.02 \\
(0.40)\end{array}$ & $\begin{array}{c}0.90 \\
(0.08)\end{array}$ & $\begin{array}{c}0.80 \\
(0.18)\end{array}$ & $\begin{array}{c}1.00 \\
(0.09)\end{array}$ & $\begin{array}{c}0.92 \\
(0.26)\end{array}$ & $\begin{array}{c}1.10 \\
(0.22)\end{array}$ \\
\hline March & $\begin{array}{c}22.82^{* * *} \\
(6.18)\end{array}$ & $\begin{array}{l}-0.35 \\
(2.94)\end{array}$ & $\begin{array}{l}-6.82 \\
(4.84)\end{array}$ & $\begin{array}{c}3.62 \\
(9.35)\end{array}$ & $\begin{array}{l}-11.09 \\
(8.55)\end{array}$ & $\begin{array}{c}1.42 \\
(3.14)\end{array}$ & $\begin{array}{c}3.98 \\
(7.75)\end{array}$ & $\begin{array}{c}1.80^{* * *} \\
(0.28)\end{array}$ & $\begin{array}{c}1.00 \\
(0.34)\end{array}$ & $\begin{array}{c}0.91 \\
(0.08)\end{array}$ & $\begin{array}{c}1.06 \\
(0.20)\end{array}$ & $\begin{array}{c}0.88 \\
(0.08)\end{array}$ & $\begin{array}{c}1.13 \\
(0.32)\end{array}$ & $\begin{array}{c}1.12 \\
(0.20)\end{array}$ \\
\hline April & $\begin{array}{c}46.03 * * * \\
(6.68)\end{array}$ & $\begin{array}{l}-4.53 \\
(2.54)\end{array}$ & $\begin{array}{l}-2.41 \\
(3.89)\end{array}$ & $\begin{array}{c}-16.48^{* *} \\
(5.37)\end{array}$ & $\begin{array}{l}-0.57 \\
(6.83)\end{array}$ & $\begin{array}{l}-2.56 \\
(2.34)\end{array}$ & $\begin{array}{l}-1.95 \\
(5.21)\end{array}$ & $\begin{array}{c}2.20 * * * \\
(0.24)\end{array}$ & $\begin{array}{c}0.68 \\
(0.14)\end{array}$ & $\begin{array}{c}0.95 \\
(0.07)\end{array}$ & $\begin{array}{c}0.55^{* * *} \\
(0.10)\end{array}$ & $\begin{array}{c}0.97 \\
(0.07)\end{array}$ & $\begin{array}{c}0.71 \\
(0.19)\end{array}$ & $\begin{array}{c}1.23 \\
(0.29)\end{array}$ \\
\hline May & $\begin{array}{c}47.94 * * * \\
(11.22)\end{array}$ & $\begin{array}{l}-3.45 \\
(4.21)\end{array}$ & $\begin{array}{c}2.09 \\
(5.94)\end{array}$ & $\begin{array}{c}0.69 \\
(7.85)\end{array}$ & $\begin{array}{l}-14.90 \\
(10.33)\end{array}$ & $\begin{array}{l}-3.63 \\
(2.74)\end{array}$ & $\begin{array}{l}-5.48 \\
(7.21)\end{array}$ & $\begin{array}{c}2.19 * * * \\
(0.32)\end{array}$ & $\begin{array}{c}0.77 \\
(0.28)\end{array}$ & $\begin{array}{c}1.14 \\
(0.15)\end{array}$ & $\begin{array}{c}1.02 \\
(0.22)\end{array}$ & $\begin{array}{c}0.81 \\
(0.11)\end{array}$ & $\begin{array}{c}0.57 \\
(0.20)\end{array}$ & $\begin{array}{c}0.98 \\
(0.35)\end{array}$ \\
\hline June & $\begin{array}{c}72.53 \\
(34.72)\end{array}$ & $\begin{array}{l}-9.33 \\
(4.10)\end{array}$ & $\begin{array}{c}-23.67 * * \\
(8.85)\end{array}$ & $\begin{array}{c}20.43 \\
(34.66)\end{array}$ & $\begin{array}{c}22.96 \\
(22.42)\end{array}$ & $\begin{array}{l}-4.98 \\
(3.08)\end{array}$ & $\begin{array}{c}-15.02 * * \\
(5.61)\end{array}$ & $\begin{array}{c}2.66^{* * *} \\
(0.77)\end{array}$ & $\begin{array}{c}0.38 \\
(0.22)\end{array}$ & $\begin{array}{l}0.50^{*} \\
(0.15)\end{array}$ & $\begin{array}{c}1.72 \\
(1.22)\end{array}$ & $\begin{array}{c}1.26 \\
(0.27)\end{array}$ & $\begin{array}{c}0.41 \\
(0.26)\end{array}$ & $\begin{array}{c}0.23 \\
(0.18)\end{array}$ \\
\hline July & $\begin{array}{l}-1.53 \\
(6.10)\end{array}$ & $\begin{array}{l}-2.82 \\
(2.46)\end{array}$ & $\begin{array}{c}-35.93 * * * \\
(4.12)\end{array}$ & $\begin{array}{c}-12.03 * \\
(6.62)\end{array}$ & $\begin{array}{c}-76.16^{* * *} \\
(6.95)\end{array}$ & $\begin{array}{c}-7.35^{* *} \\
(2.54)\end{array}$ & $\begin{array}{c}-15.27^{* *} \\
(5.40)\end{array}$ & $\begin{array}{c}1.05 \\
(0.13)\end{array}$ & $\begin{array}{c}0.68 \\
(0.15)\end{array}$ & $\begin{array}{c}0.29 * * * \\
(0.02)\end{array}$ & $\begin{array}{c}0.59 * * \\
(0.11)\end{array}$ & $\begin{array}{c}0.00 * * * \\
(0.00)\end{array}$ & $\begin{array}{c}0.00 * * * \\
(0.00)\end{array}$ & $\begin{array}{c}0.00 * * * \\
(0.00)\end{array}$ \\
\hline August & $\begin{array}{c}23.34 \\
(11.04)\end{array}$ & $\begin{array}{l}-0.63 \\
(4.90)\end{array}$ & $\begin{array}{c}-25.98^{* *} \\
(8.70)\end{array}$ & $\begin{array}{l}-10.24 \\
(9.05)\end{array}$ & $\begin{array}{c}19.07 \\
(16.28)\end{array}$ & $\begin{array}{l}-4.48 \\
(4.75)\end{array}$ & $\begin{array}{c}5.33 \\
(14.08)\end{array}$ & $\begin{array}{c}1.57 \\
(0.31)\end{array}$ & $\begin{array}{c}0.83 \\
(0.31)\end{array}$ & $\begin{array}{l}0.58^{*} \\
(0.13)\end{array}$ & $\begin{array}{c}0.79 \\
(0.25)\end{array}$ & $\begin{array}{c}1.15 \\
(0.16)\end{array}$ & $\begin{array}{c}0.69 \\
(0.38)\end{array}$ & $\begin{array}{l}1.28 \\
(0.59)\end{array}$ \\
\hline September & $\begin{array}{c}27.53^{* * *} \\
(4.53)\end{array}$ & $\begin{array}{l}-0.14 \\
(2.32)\end{array}$ & $\begin{array}{l}-2.41 \\
(3.82)\end{array}$ & $\begin{array}{l}-7.72 \\
(5.39)\end{array}$ & $\begin{array}{l}-1.13 \\
(6.90)\end{array}$ & $\begin{array}{l}-1.79 \\
(2.13)\end{array}$ & $\begin{array}{l}-5.17 \\
(4.75)\end{array}$ & $\begin{array}{c}1.74 * * * \\
(0.18)\end{array}$ & $\begin{array}{c}0.98 \\
(0.19)\end{array}$ & $\begin{array}{c}0.91 \\
(0.06)\end{array}$ & $\begin{array}{c}0.83 \\
(0.12)\end{array}$ & $\begin{array}{c}0.95 \\
(0.07)\end{array}$ & $\begin{array}{c}0.75 \\
(0.17)\end{array}$ & $\begin{array}{c}1.04 \\
(0.17)\end{array}$ \\
\hline October & $\begin{array}{c}24.79 * * \\
(8.29)\end{array}$ & $\begin{array}{l}-1.33 \\
(2.34)\end{array}$ & $\begin{array}{l}-4.08 \\
(3.77)\end{array}$ & $\begin{array}{l}-4.77 \\
(5.61)\end{array}$ & $\begin{array}{l}-7.07 \\
(6.27)\end{array}$ & $\begin{array}{l}-0.22 \\
(2.23)\end{array}$ & $\begin{array}{l}-5.27 \\
(5.04)\end{array}$ & $\begin{array}{c}1.70 * * * \\
(0.24)\end{array}$ & $\begin{array}{c}0.79 \\
(0.15)\end{array}$ & $\begin{array}{c}0.89 \\
(0.06)\end{array}$ & $\begin{array}{c}0.88 \\
(0.12)\end{array}$ & $\begin{array}{c}0.91 \\
(0.06)\end{array}$ & $\begin{array}{c}0.93 \\
(0.20)\end{array}$ & $\begin{array}{c}0.94 \\
(0.16)\end{array}$ \\
\hline November & $\begin{array}{c}6.41 \\
(4.53)\end{array}$ & $\begin{array}{l}-0.75 \\
(2.28)\end{array}$ & $\begin{array}{l}-2.56 \\
(3.74)\end{array}$ & $\begin{array}{l}-2.85 \\
(5.80)\end{array}$ & $\begin{array}{c}4.13 \\
(6.65)\end{array}$ & $\begin{array}{l}5.76^{*} \\
(2.46)\end{array}$ & $\begin{array}{c}2.24 \\
(6.25)\end{array}$ & $\begin{array}{c}1.25 \\
(0.14)\end{array}$ & $\begin{array}{c}0.82 \\
(0.16)\end{array}$ & $\begin{array}{c}0.93 \\
(0.07)\end{array}$ & $\begin{array}{c}0.97 \\
(0.14)\end{array}$ & $\begin{array}{c}1.02 \\
(0.07)\end{array}$ & $\begin{array}{l}1.61^{*} \\
(0.32)\end{array}$ & $\begin{array}{c}1.12 \\
(0.21)\end{array}$ \\
\hline December & $\begin{array}{c}1.50 \\
(5.04)\end{array}$ & $\begin{array}{c}0.27 \\
(2.48)\end{array}$ & $\begin{array}{l}-4.52 \\
(4.22)\end{array}$ & $\begin{array}{l}-1.49 \\
(6.50)\end{array}$ & $\begin{array}{c}9.99 \\
(7.13)\end{array}$ & $\begin{array}{c}0.75 \\
(2.79)\end{array}$ & $\begin{array}{l}-1.91 \\
(5.83)\end{array}$ & $\begin{array}{c}1.07 \\
(0.15)\end{array}$ & $\begin{array}{c}0.94 \\
(0.21)\end{array}$ & $\begin{array}{c}0.91 \\
(0.07)\end{array}$ & $\begin{array}{c}1.08 \\
(0.17)\end{array}$ & $\begin{array}{c}1.09 \\
(0.07)\end{array}$ & $\begin{array}{c}1.04 \\
(0.25)\end{array}$ & $\begin{array}{c}0.94 \\
(0.16)\end{array}$ \\
\hline
\end{tabular}


Time Diary Completed:

Omitted=During Day

End of diary day

$\begin{array}{ccccccc}0.26 & -0.80 & 3.38 & -1.00 & -11.67^{* *} & -2.27 & 5.78 \\ (4.99) & (1.33) & (2.39) & (3.42) & (4.16) & (1.80) & (3.26) \\ -3.14 & 0.57 & 2.96 & 2.73 & -5.44 & -3.59 & 6.70 \\ (4.51) & (1.79) & (2.80) & (4.41) & (5.82) & (1.95) & (4.23) \\ -2.19 & -2.01 & 0.26 & 1.76 & -2.81 & -3.72 & -0.50 \\ (6.20) & (1.94) & (3.40) & (5.72) & (6.99) & (2.51) & (5.14) \\ -19.54 & 3.57 & 4.82 & 14.49 & -7.69 & -4.10 & 10.72 \\ (9.70) & (4.08) & (7.59) & (8.51) & (13.45) & (3.49) & (8.88)\end{array}$

$\begin{array}{ccccccc}0.99 & 0.96 & 1.10 & 0.96 & 0.89 * * * & 0.79 & 1.19 \\ (0.09) & (0.11) & (0.05) & (0.09) & (0.04) & (0.12) & (0.15) \\ 0.95 & 1.12 & 1.10 & 1.04 & 0.96 & 0.77 & 1.22 \\ (0.09) & (0.15) & (0.06) & (0.12) & (0.05) & (0.13) & (0.19) \\ 1.05 & 0.80 & 1.05 & 1.02 & 0.96 & 0.69 & 1.09 \\ (0.13) & (0.15) & (0.08) & (0.16) & (0.07) & (0.15) & (0.24) \\ 0.65 & 1.31 & 1.17 & 1.52 & 0.92 & 0.61 & 1.19 \\ (0.17) & (0.48) & (0.17) & (0.39) & (0.11) & (0.35) & (0.39)\end{array}$

Was Diary Completed with

Child? Omitted=Yes

\begin{tabular}{|c|c|c|c|c|c|c|c|c|c|c|c|c|c|c|}
\hline Parent only & $\begin{array}{c}4.92 \\
(4.25)\end{array}$ & $\begin{array}{l}-0.57 \\
(1.17)\end{array}$ & $\begin{array}{l}5.81^{* *} \\
(2.03)\end{array}$ & $\begin{array}{l}-2.00 \\
(2.85)\end{array}$ & $\begin{array}{l}-5.41 \\
(3.67)\end{array}$ & $\begin{array}{c}0.63 \\
(1.33)\end{array}$ & $\begin{array}{l}-0.08 \\
(3.00)\end{array}$ & $\begin{array}{c}1.12 \\
(0.08)\end{array}$ & $\begin{array}{c}0.83 \\
(0.09)\end{array}$ & $\begin{array}{l}1.12^{* *} \\
(0.04)\end{array}$ & $\begin{array}{c}0.96 \\
(0.08)\end{array}$ & $\begin{array}{c}0.95 \\
(0.04)\end{array}$ & $\begin{array}{c}1.08 \\
(0.13)\end{array}$ & $\begin{array}{l}0.88 \\
(0.09)\end{array}$ \\
\hline Don't know & $\begin{array}{l}15.92 \\
(9.96)\end{array}$ & $\begin{array}{l}-4.75 \\
(4.02)\end{array}$ & $\begin{array}{l}-0.64 \\
(7.61)\end{array}$ & $\begin{array}{l}-8.31 \\
(8.82)\end{array}$ & $\begin{array}{c}-0.86 \\
(13.78)\end{array}$ & $\begin{array}{c}1.01 \\
(3.37)\end{array}$ & $\begin{array}{l}-2.70 \\
(9.29)\end{array}$ & $\begin{array}{c}1.35 \\
(0.36)\end{array}$ & $\begin{array}{c}0.58 \\
(0.22)\end{array}$ & $\begin{array}{c}1.00 \\
(0.14)\end{array}$ & $\begin{array}{c}0.78 \\
(0.20)\end{array}$ & $\begin{array}{c}0.99 \\
(0.12)\end{array}$ & $\begin{array}{c}1.15 \\
(0.66)\end{array}$ & $\begin{array}{c}1.11 \\
(0.37)\end{array}$ \\
\hline Constant & $\begin{array}{c}46.15^{* * *} \\
(7.82)\end{array}$ & $\begin{array}{c}15.31^{* * *} \\
(2.63)\end{array}$ & $\begin{array}{c}52.83^{* * *} \\
(4.41)\end{array}$ & $\begin{array}{c}31.36^{* * *} \\
(5.56)\end{array}$ & $\begin{array}{c}84.99 * * * \\
(7.47)\end{array}$ & $\begin{array}{c}9.79 * * * \\
(2.85)\end{array}$ & $\begin{array}{l}15.67 \\
(6.74)\end{array}$ & $\begin{array}{c}40.61^{* * *} \\
(5.34)\end{array}$ & $\begin{array}{c}17.18^{* * *} \\
(3.75)\end{array}$ & $\begin{array}{c}52.19^{* * *} \\
(4.48)\end{array}$ & $\begin{array}{c}30.12^{* * *} \\
(5.22)\end{array}$ & $\begin{array}{c}86.86^{* * *} \\
(7.29)\end{array}$ & $\begin{array}{c}8.23^{* * *} \\
(2.42)\end{array}$ & $\begin{array}{c}14.67^{* * *} \\
(4.22)\end{array}$ \\
\hline Observations & 3,574 & 3,574 & 3,574 & 3,574 & 3,574 & 3,574 & 3,574 & 3,574 & 3,574 & 3,574 & 3,574 & 3,574 & 3,574 & 3,574 \\
\hline R-Squared & 0.12 & 0.07 & 0.15 & 0.03 & 0.06 & 0.02 & 0.11 & & & & & & & \\
\hline
\end{tabular}

Notes: Clustered errors in parentheses. P-value alpha values of 0.01, 0.05, and 0.1 adjusted for multiple hypothesis testing using D/AP procedure according to the Bonferroni test with

correlation $=0.15$ resulting in p-value cut-offs: $* * * p<0.0019, * * p<0.0096, * p<0.02$ 
Table A10 Linear regression results for time spent in activities for girls (panel A) and boys (panel B) including both maternal education and income -with and without additional controls

\begin{tabular}{|c|c|c|c|c|c|c|c|c|c|c|c|c|c|c|}
\hline & $\begin{array}{l}\quad(1) \\
\text { Sport } \\
\text { (Min) } \\
\end{array}$ & $\begin{array}{l}\quad(2) \\
\text { Sport } \\
\text { (Min) } \\
\end{array}$ & $\begin{array}{l}\quad(3) \\
\text { Reading } \\
\text { (Min) }\end{array}$ & $\begin{array}{l}\quad(4) \\
\text { Reading } \\
\text { (Min) }\end{array}$ & $\begin{array}{l}\quad(5) \\
\text { Homework } \\
\text { (Min) }\end{array}$ & $\begin{array}{l}\quad(6) \\
\text { Homework } \\
\text { (Min) }\end{array}$ & $\begin{array}{l}\text { (7) } \\
\text { Playing } \\
\text { (Min) }\end{array}$ & $\begin{array}{l}\text { (8) } \\
\text { Playing } \\
\text { (Min) }\end{array}$ & $\begin{array}{l}\text { (9) } \\
\text { Media } \\
\text { (Min) } \\
\end{array}$ & $\begin{array}{c}\text { (10) } \\
\text { Media (Min) }\end{array}$ & $\begin{array}{l}\quad(11) \\
\text { Hobbies } \\
\text { (Min) }\end{array}$ & $\begin{array}{l}\begin{array}{l}(12) \\
\text { Hobbies } \\
\text { (Min) }\end{array} \\
\end{array}$ & $\begin{array}{l}\text { (13) } \\
\text { Family } \\
\text { (Min) }\end{array}$ & $\begin{array}{l}(14) \\
\text { Family } \\
\text { (Min) }\end{array}$ \\
\hline Panel A: Girls & & & & & & & & & & & & & & \\
\hline $\begin{array}{l}\text { Maternal } \\
\text { Education }\end{array}$ & $\begin{array}{c}-37.19 * * * \\
(4.65)\end{array}$ & $\begin{array}{c}-36.88^{* * *} \\
(4.67)\end{array}$ & $\begin{array}{c}-8.81^{* *} \\
(3.03)\end{array}$ & $\begin{array}{c}-8.47^{* *} \\
(3.00)\end{array}$ & $\begin{array}{c}34.80^{* * *} \\
(7.71)\end{array}$ & $\begin{array}{c}34.85^{* * *} \\
(7.69)\end{array}$ & $\begin{array}{l}12.67 \\
(8.48)\end{array}$ & $\begin{array}{l}11.90 \\
(8.43)\end{array}$ & $\begin{array}{c}36.51 * * * \\
(10.80)\end{array}$ & $\begin{array}{c}36.51^{* * *} \\
(10.74)\end{array}$ & $\begin{array}{c}5.81 \\
(5.80)\end{array}$ & $\begin{array}{c}5.84 \\
(5.79)\end{array}$ & $\begin{array}{c}29.15^{* * *} \\
(6.68)\end{array}$ & $\begin{array}{c}28.98^{* * *} \\
(6.65)\end{array}$ \\
\hline Secondary & $\begin{array}{l}-3.01 \\
(4.35)\end{array}$ & $\begin{array}{l}-2.83 \\
(4.36)\end{array}$ & $\begin{array}{c}4.85 \\
(2.41)\end{array}$ & $\begin{array}{c}5.02 \\
(2.41)\end{array}$ & $\begin{array}{l}-0.63 \\
(2.63)\end{array}$ & $\begin{array}{l}-0.77 \\
(2.67)\end{array}$ & $\begin{array}{l}0.52 \\
(4.55)\end{array}$ & $\begin{array}{c}0.00 \\
(4.52)\end{array}$ & $\begin{array}{l}4.08 \\
(4.42)\end{array}$ & $\begin{array}{c}4.63 \\
(4.45)\end{array}$ & $\begin{array}{c}2.81 \\
(2.71)\end{array}$ & $\begin{array}{c}2.72 \\
(2.72)\end{array}$ & $\begin{array}{c}4.37 \\
(3.13)\end{array}$ & $\begin{array}{c}3.50 \\
(3.14)\end{array}$ \\
\hline $\begin{array}{l}\text { More than } \\
\text { secondary }\end{array}$ & $\begin{array}{l}-8.32 \\
(4.52)\end{array}$ & $\begin{array}{l}-7.48 \\
(4.56)\end{array}$ & $\begin{array}{c}7.63 * * * \\
(2.24)\end{array}$ & $\begin{array}{c}7.45^{* * *} \\
(2.25)\end{array}$ & $\begin{array}{l}-2.95 \\
(2.48)\end{array}$ & $\begin{array}{l}-2.20 \\
(2.59)\end{array}$ & $\begin{array}{l}-0.16 \\
(4.39)\end{array}$ & $\begin{array}{l}-0.57 \\
(4.38)\end{array}$ & $\begin{array}{l}-0.40 \\
(4.32)\end{array}$ & $\begin{array}{l}-0.53 \\
(4.48)\end{array}$ & $\begin{array}{l}6.23 * \\
(2.61)\end{array}$ & $\begin{array}{c}5.96 \\
(2.66)\end{array}$ & $\begin{array}{c}2.10 \\
(2.99)\end{array}$ & $\begin{array}{c}1.04 \\
(3.02)\end{array}$ \\
\hline Secondary * & & & & & & & & & & & & & & \\
\hline Wave 2 & $\begin{array}{c}9.61 \\
(5.57)\end{array}$ & $\begin{array}{l}9.73 \\
(5.62)\end{array}$ & $\begin{array}{l}-2.73 \\
(3.32)\end{array}$ & $\begin{array}{l}-2.65 \\
(3.27)\end{array}$ & $\begin{array}{c}0.86 \\
(7.82)\end{array}$ & $\begin{array}{c}1.41 \\
(7.82)\end{array}$ & $\begin{array}{l}-15.83 \\
(8.82)\end{array}$ & $\begin{array}{l}-15.20 \\
(8.81)\end{array}$ & $\begin{array}{c}-9.31 \\
(11.31)\end{array}$ & $\begin{array}{c}-9.76 \\
(11.31)\end{array}$ & $\begin{array}{l}-7.16 \\
(5.76)\end{array}$ & $\begin{array}{l}-7.48 \\
(5.72)\end{array}$ & $\begin{array}{l}4.05 \\
(7.35)\end{array}$ & $\begin{array}{c}3.59 \\
(7.31)\end{array}$ \\
\hline $\begin{array}{l}\text { More than } \\
\text { Secondary * }\end{array}$ & & & & & & & & & & & & & & \\
\hline Wave 2 & $\begin{array}{c}22.10^{* * *} \\
(5.78)\end{array}$ & $\begin{array}{c}21.94 * * * \\
(5.78)\end{array}$ & $\begin{array}{l}-0.40 \\
(3.43)\end{array}$ & $\begin{array}{l}-0.08 \\
(3.40)\end{array}$ & $\begin{array}{l}-0.07 \\
(7.98)\end{array}$ & $\begin{array}{l}-0.08 \\
(8.02)\end{array}$ & $\begin{array}{l}-18.24 \\
(9.06)\end{array}$ & $\begin{array}{l}-17.87 \\
(9.07)\end{array}$ & $\begin{array}{l}-15.01 \\
(11.50)\end{array}$ & $\begin{array}{l}-14.70 \\
(11.51)\end{array}$ & $\begin{array}{l}-8.07 \\
(5.98)\end{array}$ & $\begin{array}{l}-8.34 \\
(6.01)\end{array}$ & $\begin{array}{l}-1.51 \\
(7.17)\end{array}$ & $\begin{array}{l}-2.32 \\
(7.11)\end{array}$ \\
\hline Log income & $\begin{array}{l}2.05 \\
(6.05)\end{array}$ & $\begin{array}{l}0.67 \\
(6.25)\end{array}$ & $\begin{array}{l}-1.59 \\
(1.88)\end{array}$ & $\begin{array}{l}-0.00 \\
(1.88)\end{array}$ & $\begin{array}{l}-0.82 \\
(1.85)\end{array}$ & $\begin{array}{l}-2.30 \\
(2.11)\end{array}$ & $\begin{array}{l}-1.12 \\
(3.34)\end{array}$ & $\begin{array}{c}0.52 \\
(3.47)\end{array}$ & $\begin{array}{l}-2.05 \\
(3.34)\end{array}$ & $\begin{array}{l}-0.65 \\
(3.75)\end{array}$ & $\begin{array}{l}0.38 \\
(1.98)\end{array}$ & $\begin{array}{c}0.08 \\
(2.18)\end{array}$ & $\begin{array}{c}0.12 \\
(2.42)\end{array}$ & $\begin{array}{l}-2.04 \\
(2.73)\end{array}$ \\
\hline $\begin{array}{l}\text { Log income * } \\
\text { Wave } 2\end{array}$ & $\begin{array}{c}0.33 \\
(6.66)\end{array}$ & $\begin{array}{c}0.25 \\
(6.49)\end{array}$ & $\begin{array}{c}0.53 \\
(3.02)\end{array}$ & $\begin{array}{c}0.63 \\
(3.01)\end{array}$ & $\begin{array}{c}5.04 \\
(4.93)\end{array}$ & $\begin{array}{c}4.30 \\
(5.02)\end{array}$ & $\begin{array}{l}-1.48 \\
(6.37)\end{array}$ & $\begin{array}{l}-2.27 \\
(6.26)\end{array}$ & $\begin{array}{c}3.47 \\
(7.61)\end{array}$ & $\begin{array}{c}4.36 \\
(7.62)\end{array}$ & $\begin{array}{c}2.72 \\
(3.21)\end{array}$ & $\begin{array}{c}2.94 \\
(3.23)\end{array}$ & $\begin{array}{l}-1.34 \\
(5.13)\end{array}$ & $\begin{array}{l}-1.75 \\
(5.15)\end{array}$ \\
\hline Constant & $\begin{array}{c}33.70^{* * *} \\
(5.92)\end{array}$ & $\begin{array}{c}30.79 \\
(14.06)\end{array}$ & $\begin{array}{c}21.98^{* * *} \\
(4.18)\end{array}$ & $\begin{array}{c}-7.95 \\
(24.82)\end{array}$ & $\begin{array}{c}53.16^{* * *} \\
(4.71)\end{array}$ & $\begin{array}{c}76.63 * * * \\
(20.72)\end{array}$ & $\begin{array}{c}40.60 * * * \\
(7.46)\end{array}$ & $\begin{array}{c}62.96 \\
(29.22)\end{array}$ & $\begin{array}{c}84.92^{* * *} \\
(8.67)\end{array}$ & $\begin{array}{c}125.25^{* * *} \\
(13.62)\end{array}$ & $\begin{array}{c}18.24^{* * *} \\
(4.86)\end{array}$ & $\begin{array}{l}-10.12 \\
(33.22)\end{array}$ & $\begin{array}{c}9.10 \\
(5.40)\end{array}$ & $\begin{array}{l}-41.22 \\
(21.82)\end{array}$ \\
\hline $\begin{array}{l}\text { Adjusted for } \\
\text { covariates }\end{array}$ & N & $Y$ & $N$ & $Y$ & N & Y & $\mathrm{N}$ & $Y$ & $\mathrm{~N}$ & $Y$ & $N$ & $Y$ & $\mathrm{~N}$ & $Y$ \\
\hline Observations & 3,339 & 3,338 & 3,339 & 3,338 & 3,339 & 3,338 & 3,339 & 3,338 & 3,339 & 3,338 & 3,339 & 3,338 & 3,339 & 3,338 \\
\hline
\end{tabular}




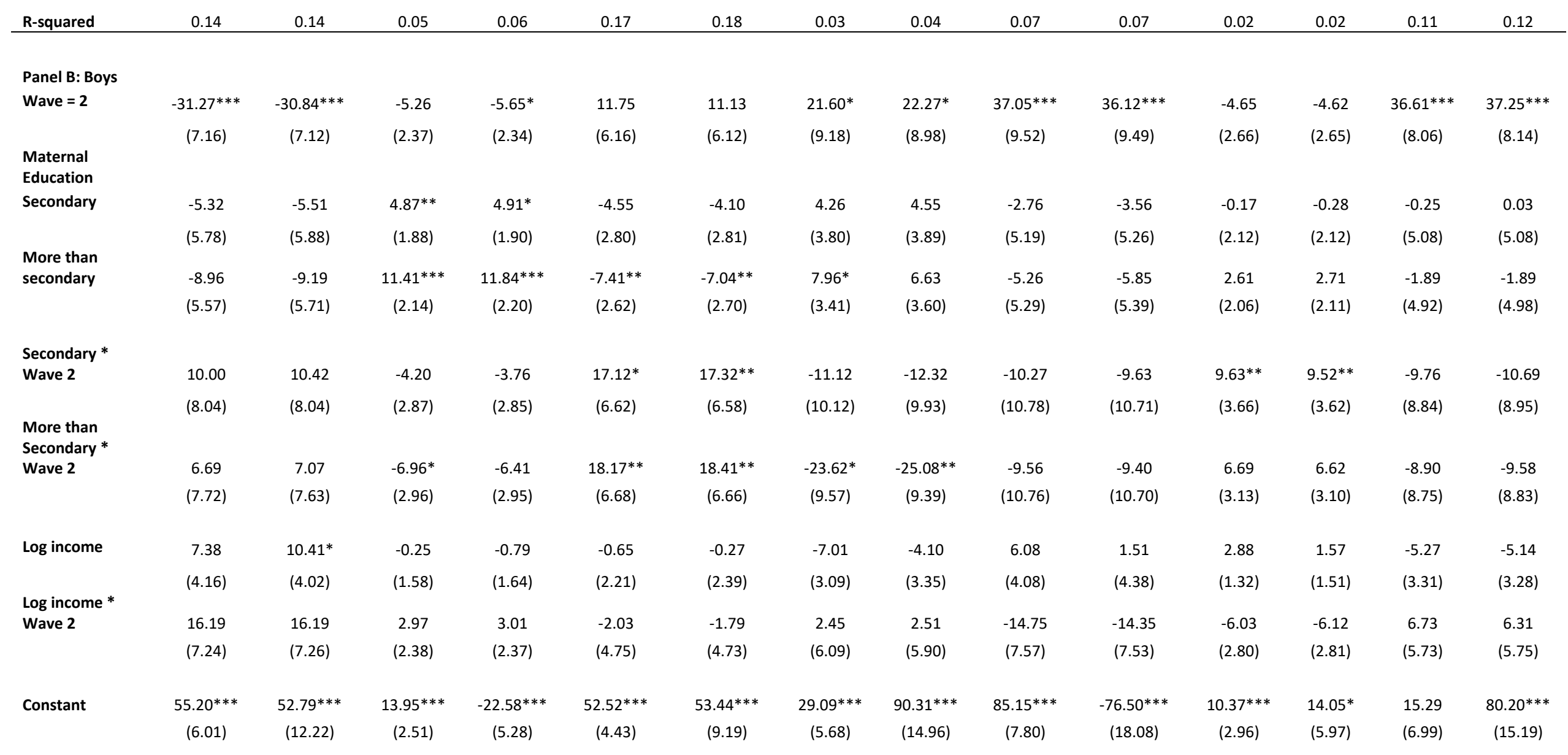

Adjusted for

\begin{tabular}{|c|c|c|c|c|c|c|c|c|c|c|c|c|c|c|}
\hline covariates & N & $Y$ & $\mathrm{~N}$ & $Y$ & N & $Y$ & N & $Y$ & $\mathrm{~N}$ & $Y$ & $\mathrm{~N}$ & $Y$ & $\mathrm{~N}$ & $Y$ \\
\hline Observations & 3,340 & 3,340 & 3,340 & 3,340 & 3,340 & 3,340 & 3,340 & 3,340 & 3,340 & 3,340 & 3,340 & 3,340 & 3,340 & 3,340 \\
\hline R-squared & 0.16 & 0.16 & 0.06 & 0.07 & 0.15 & 0.16 & 0.04 & 0.06 & 0.07 & 0.07 & 0.02 & 0.03 & 0.10 & 0.11 \\
\hline
\end{tabular}

Notes: Clustered errors in parentheses. Unadjusted regression include diary characteristics. Adjusted regression additionally include mother's and father's employment, mother's marital

status, and household size. P-value alpha values of $0.01,0.05$, and 0.1 adjusted for multiple hypothesis testing using D/AP procedure according to the Bonferroni test with

correlation $=0.15$ resulting in $p$-value cut-offs: $* * * p<0.0019, * * p<0.0096, * p<0.02$ 
Table A11 Linear regression results for time spent in activities for girls and boys including both maternal education and income - with all additional controls

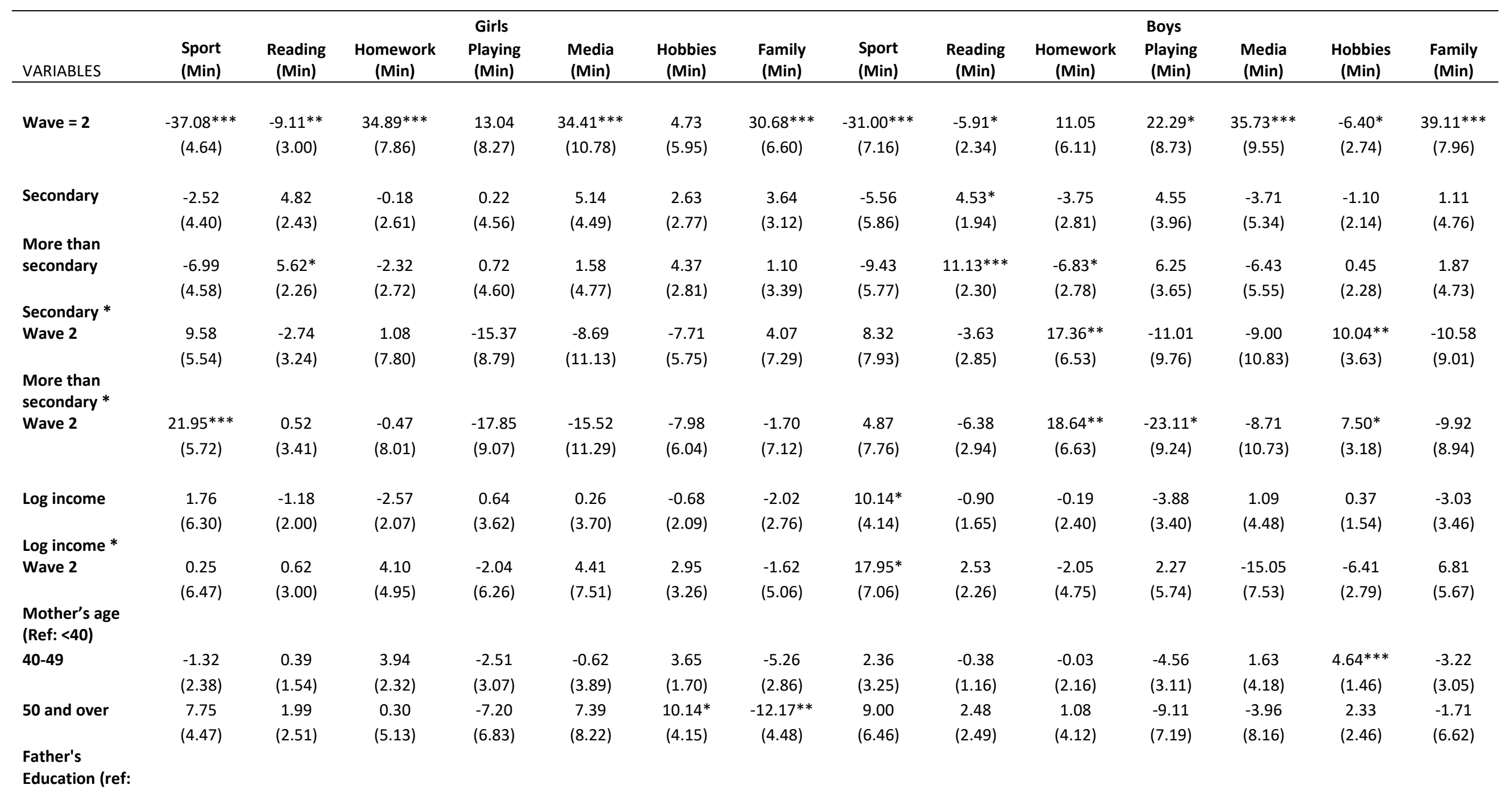


Less than

secondary)

Secondary

$\begin{array}{llll}-1.48 & 1.71 & -2.77 & -0.61\end{array}$

secondary

$$
\text { (3.06) }
$$

$$
-0.61
$$

$-4.12$

0.77

$-2.42$

$-3.82$

$\begin{array}{cc}3.56 & -3.09 \\ (1.63) & (2.97) \\ & \\ 3.35^{*} & -1.09 \\ (1.42) & (3.17) \\ & \\ 37.24^{* * *} & 0.77 \\ (3.78) & (7.96) \\ 0.17 & -2.95 \\ (2.18) & (4.19)\end{array}$

1.94
$(3.71)$
3.38
$(3.32)$

1.37
$(5.01)$

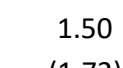

$(3.32)$
-
$51.21 * * *$

household

(3.24)

$5.88 * * *$

$-1.62$

-1.10
$(3.79)$

$-8.03$

3.94

$0.14 \quad-2.57$

$51.21 * * *$

2.49
$(4.90)$

$$
\text { (1.72) }
$$

6.38
$(6.49)$

$160.34^{* * *}$

$5.18^{* *}$

(4.12)

$\begin{array}{cc}(8.02) & (8.34) \\ 1.49 & -0.79 \\ (4.45) & (2.17)\end{array}$

-7.76
$(4.76)$

9.78
$(6.97)$

-4.02
$(7.63)$

$(9.66)$
-5.01
$(3.17)$

-2.67
$(4.86)$

5.93
$(7.72)$

(2.18)

(6.49)

(8.11)

0.08
$(4.07)$

0.16
$(2.67)$

Mother's

Marital Status -

Married

$\begin{array}{ccc}5.32 & 28.50 & -49.49 * * *\end{array}$

$6.44-131.13$

\subsection{0}

71.96

$-17.9936 .66 * * *$

Father's

$$
\text { (14.73) }
$$

(26.61) (7.28)

(10.75)

(58.87)

(27.39)

(36.81)

(9.94)

(3.87)

9.36

-

Employment

Employed

5.59
$(3.64)$

$0.26 \quad 7.34$

$0.09 \quad-7.86$

$-1.97 \quad 0.36$

\subsection{1}

$-6.70$

$56.93 * * *$

$165.19 * * *$

$-4.34$

(4.43)

$65.11 * * *$

Not in

household

$-7.48$

(2.08)

(4.33)

(4.37) (6.22)

(3.44)

(4.05)

(5.24)

(1.80)

-6.70
$(4.14)$

$-9.00 \quad 5.54$

(5.47)

5.54
$(6.34)$

3.52

5.17

$\begin{array}{lllllll}-7.48 & 19.71 & 1.91 & -43.82^{* * *} & 19.04 & 19.58 & 42.81 \\ (15.11) & (25.77) & (7.89) & (12.23) & (56.88) & (26.49) & (35.99)\end{array}$

\section{Mother's}

Employment -

Employed

$\begin{array}{ll}-2.34 & -2.23 \\ (2.26) & (1.54)\end{array}$

$\begin{array}{lcc}-2.47 & 0.56 & -0.16 \\ (2.47) & (2.98) & (3.50)\end{array}$

2.10

$8.11^{* * *}$

$8.11^{* * *} \quad-2.01$

$-2.05$

$-0.34$

$8.01 * *$

(2.91)

5.77

0.33

$-0.97$

Number of

children in

household

$\begin{array}{cc}0.26 & 1.23 \\ (1.10) & (0.73)\end{array}$

-0.82
$(1.13)$

$\begin{array}{ll}-0.57 & 1.92 \\ (1.45) & (1.65)\end{array}$

$-0.49$

$\begin{array}{ll}-1.20 & 3.97^{*} \\ (1.23) & (1.53)\end{array}$

-0.56
$(0.58)$

-1.46
$(1.01)$

1.71
$(1.56)$

-1.61
$(1.91)$

$-0.89$

$-0.44$

Child health

(Ref: no

problems) 


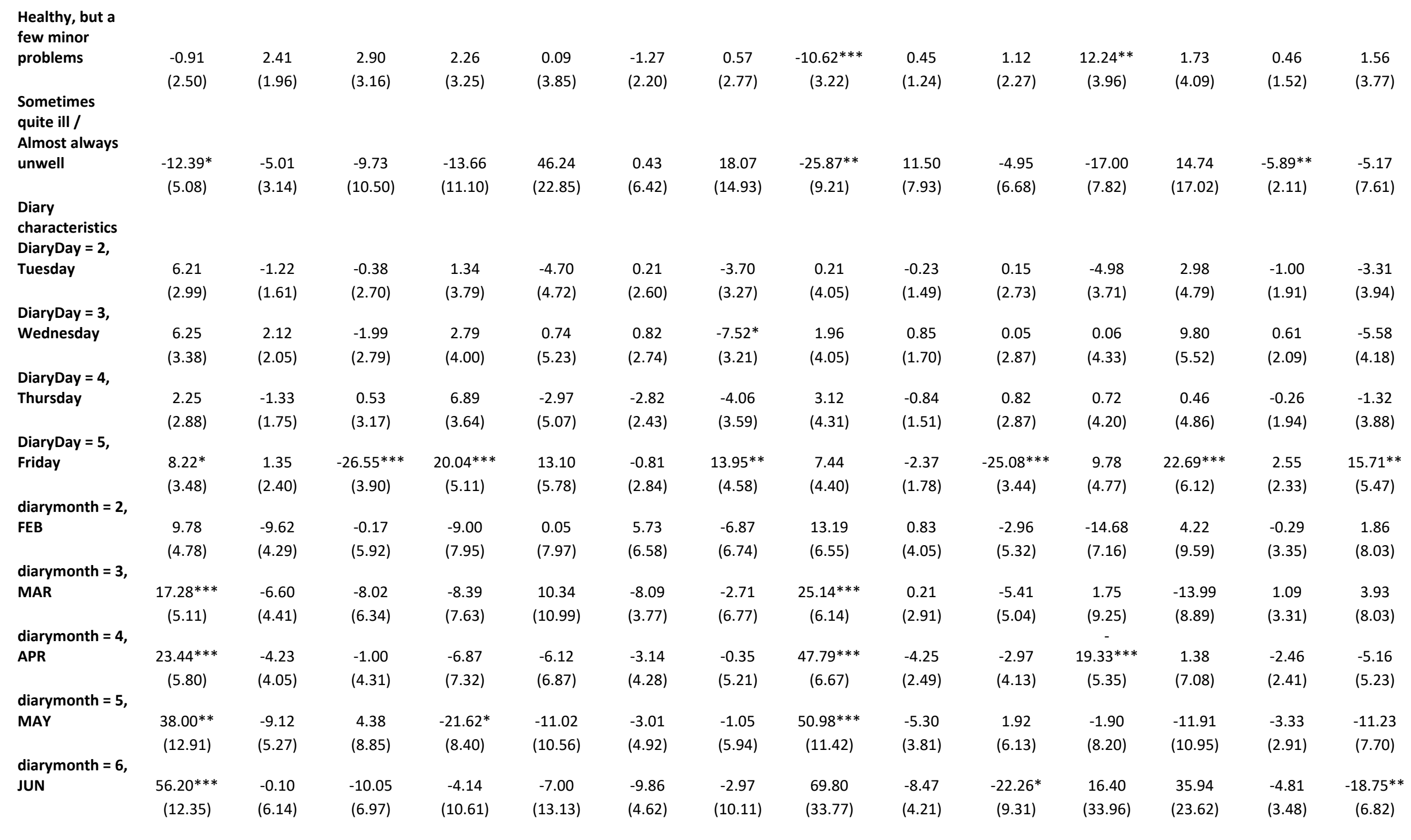




\section{diarymonth $=7$}

JUL

\section{diarymonth $=8$}

AUG

diarymonth $=9$

SEP

diarymonth $=$

10, OCT

diarymonth =

11, NOV

diarymonth =

12, DEC

When was the

Diary

Completed?

$=2$, End of diary

day

= 3, Day after

diary day

= 4, Later

5 , Don't know

Was the Diary

Completed with

the Study Child?

$=2$, parent

$-2.50$

(2.85)

0.34

(3.41)

$-3.33$

(3.98)

(13.93)

$$
\begin{gathered}
3.50 \\
(2.31)
\end{gathered}
$$

-2.31
$(1.34)$

0.17

(2.41)

$-1.31$

Was the Diary

Completed with
$-9.95$
$-11.94$

(7.01)

1.05

(2.77)

$-34.68 * * *$

(5.41)

$-8.91$

(6.80)

$-75.04 * * *$

(8.82)

$-7.45^{*}$

(3.06)

$19.87^{* * *}$

$0.37 * *$

$-0.20$

$-27.41 * * *$

$-14.71$

(8.75)

(8.94)

19.26

$\begin{array}{ll}-3.54 & -8.79\end{array}$

(3.99)

(5.49)

0.52

(4.56)

(2.34)

$-3.62$

(3.81)

$-5.13$

(4.07)

(2.26)

$-1.14$

$-2.10$

$-3.89$

0.38

7.33

6.68
$(4.50)$

(2.25)

(3.95)

$-5.10$

(4.43)

(6.37)

(4.51)

(5.69)

(5.24)

(2.45)

$-0.32$

$-3.86$

1.92

(2.21)

(3.24)

$-3.22$

(3.86)

(1.27)

3.25

(2.42)

$-1.77$

(3.53)

$-10.78 *$

1.26

3.18

(2.90)

4.42

$(2.52)$
-3.87

$-3.36$

(3.26) (4.47)
1.05

$(1.81)$
-1.41

(2.83)

(4.01)

(6.33)

$-7.50$

(5.76)

$-19.38$

(1.87)

3.75

(3.61)

5.54

(4.38)

1.39

(5.69)

11.36

(7.93)

(7.14)

$-3.33$

(6.29)

5.72

(6.87)

11.46

(7.42)

(2.85)

(5.23)

(9.11)

$(4.72)$

(4.24)

(7.85)

$7.04^{* * *}$

$-11.10 * * * \quad-3.29$

4.23

2.01

$-0.7$

(1.14)

(2.05)

$-2.40$

$-5.65$

$13.77^{*}$

14.76

$-5.04$

$-0.01$

$-5.07$

(5.31)

$-1.69$

(2.28)

$\begin{array}{ll}-0.15 & -7.07\end{array}$

(2.31) (5.10)

$4.69 \quad 1.23$

(2.52) (6.90)

$0.86 \quad-7.30$

(2.85) (5.23) 


\begin{tabular}{|c|c|c|c|c|c|c|c|c|c|c|c|c|c|c|}
\hline & (12.96) & $(5.82)$ & $(6.47)$ & (7.51) & (9.58) & $(4.70)$ & $(5.77)$ & (10.24) & (4.17) & $(7.82)$ & $(8.37)$ & (14.27) & (3.54) & (9.84) \\
\hline Constant & $\begin{array}{c}26.18 \\
(15.84)\end{array}$ & $\begin{array}{l}-10.88 \\
(26.66)\end{array}$ & $\begin{array}{c}98.89 * * * \\
(7.54)\end{array}$ & $\begin{array}{c}34.18^{* *} \\
(11.74)\end{array}$ & $\begin{array}{c}216.99 * * * \\
(58.68)\end{array}$ & $\begin{array}{l}-25.17 \\
(27.52)\end{array}$ & $\begin{array}{l}-59.89 \\
(36.87)\end{array}$ & $\begin{array}{c}69.47^{* * *} \\
(12.95)\end{array}$ & $\begin{array}{c}-23.18^{* * *} \\
(5.82)\end{array}$ & $\begin{array}{c}53.61^{* * *} \\
(9.74)\end{array}$ & $\begin{array}{c}82.42^{* * *} \\
(14.86)\end{array}$ & $\begin{array}{c}-85.32^{* * *} \\
(18.67)\end{array}$ & $\begin{array}{l}10.58 \\
(6.01)\end{array}$ & $\begin{array}{c}83.61 * * * \\
(17.22)\end{array}$ \\
\hline Observations & 3,338 & 3,338 & 3,338 & 3,338 & 3,338 & 3,338 & 3,338 & 3,340 & 3,340 & 3,340 & 3,340 & 3,340 & 3,340 & 3,340 \\
\hline R-squared & 0.14 & 0.07 & 0.18 & 0.05 & 0.09 & 0.03 & 0.13 & 0.17 & 0.08 & 0.16 & 0.07 & 0.07 & 0.04 & 0.12 \\
\hline
\end{tabular}


Table A12 Full Table for fixed effects models (Girls)

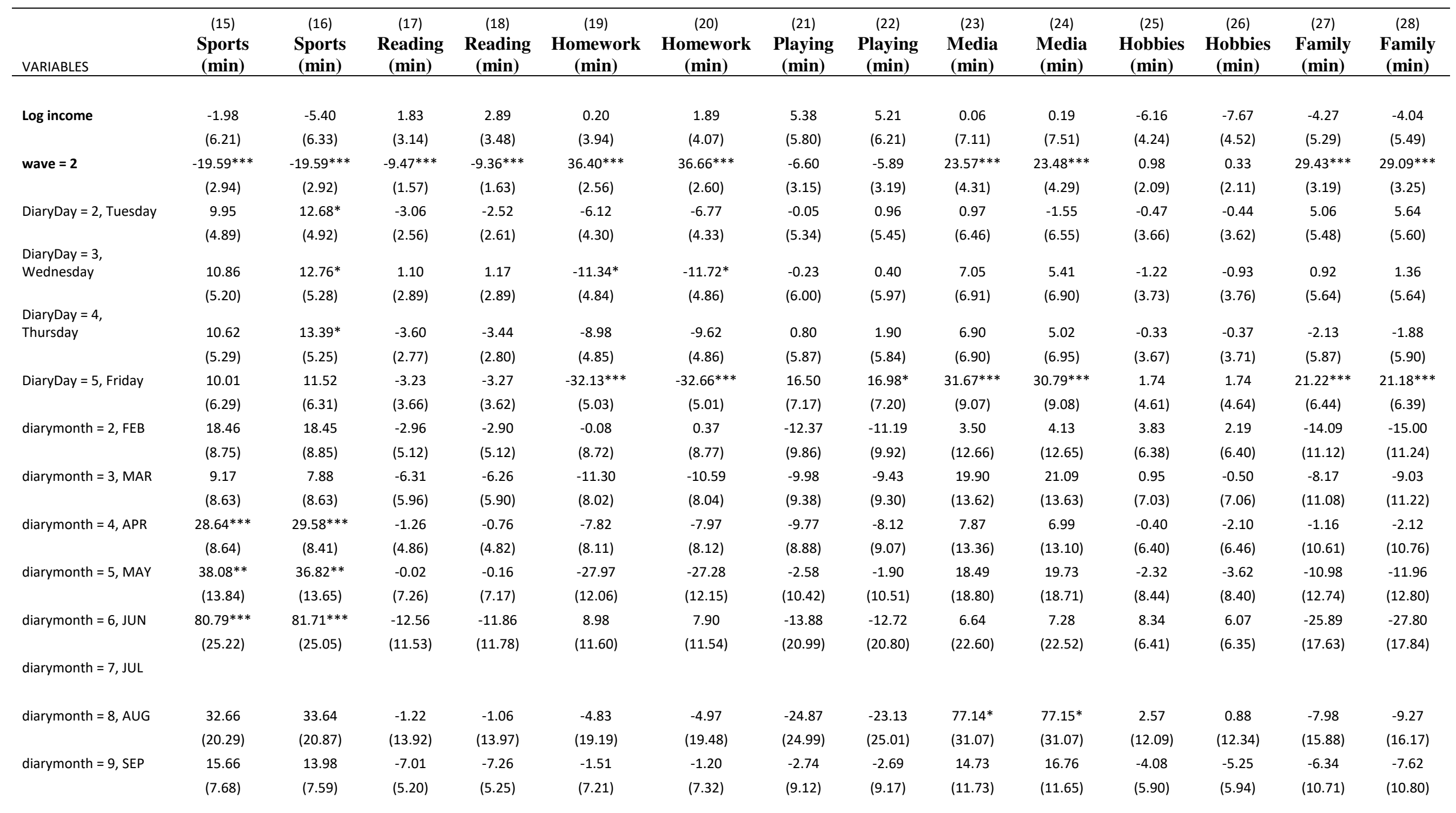




\begin{tabular}{|c|c|c|c|c|c|c|c|c|c|c|c|c|c|c|}
\hline diarymonth $=10, \mathrm{OCT}$ & $\begin{array}{l}10.31 \\
(6.99)\end{array}$ & $\begin{array}{l}8.72 \\
(6.90)\end{array}$ & $\begin{array}{l}-6.05 \\
(5.79)\end{array}$ & $\begin{array}{l}-6.32 \\
(5.87)\end{array}$ & $\begin{array}{l}-7.63 \\
(6.77)\end{array}$ & $\begin{array}{l}-7.42 \\
(6.89)\end{array}$ & $\begin{array}{l}-9.26 \\
(7.17)\end{array}$ & $\begin{array}{l}-9.19 \\
(7.27)\end{array}$ & $\begin{array}{c}18.64 \\
(10.48)\end{array}$ & $\begin{array}{c}20.63 \\
(10.41)\end{array}$ & $\begin{array}{l}-0.10 \\
(5.92)\end{array}$ & $\begin{array}{l}-1.41 \\
(5.94)\end{array}$ & $\begin{array}{c}2.52 \\
(9.69)\end{array}$ & $\begin{array}{l}1.12 \\
(9.88)\end{array}$ \\
\hline diarymonth $=11, \mathrm{NOV}$ & $\begin{array}{c}0.03 \\
(6.90)\end{array}$ & $\begin{array}{l}-0.44 \\
(6.84)\end{array}$ & $\begin{array}{l}-2.79 \\
(4.60)\end{array}$ & $\begin{array}{l}-2.94 \\
(4.59)\end{array}$ & $\begin{array}{l}-1.40 \\
(6.40)\end{array}$ & $\begin{array}{l}-1.18 \\
(6.47)\end{array}$ & $\begin{array}{l}-5.99 \\
(6.84)\end{array}$ & $\begin{array}{l}-5.08 \\
(6.91)\end{array}$ & $\begin{array}{c}15.85 \\
(10.56)\end{array}$ & $\begin{array}{c}16.94 \\
(10.43)\end{array}$ & $\begin{array}{c}1.99 \\
(5.59)\end{array}$ & $\begin{array}{c}0.40 \\
(5.66)\end{array}$ & $\begin{array}{c}6.54 \\
(9.68)\end{array}$ & $\begin{array}{l}5.21 \\
(9.82)\end{array}$ \\
\hline diarymonth $=12, \mathrm{DEC}$ & $\begin{array}{l}-3.14 \\
(7.63)\end{array}$ & $\begin{array}{l}-4.07 \\
(7.49)\end{array}$ & $\begin{array}{l}-5.04 \\
(5.08)\end{array}$ & $\begin{array}{l}-4.89 \\
(5.03)\end{array}$ & $\begin{array}{l}-8.53 \\
(7.02)\end{array}$ & $\begin{array}{l}-7.94 \\
(7.02)\end{array}$ & $\begin{array}{l}-4.59 \\
(7.44)\end{array}$ & $\begin{array}{l}-3.96 \\
(7.42)\end{array}$ & $\begin{array}{c}19.99 \\
(12.50)\end{array}$ & $\begin{array}{c}20.69 \\
(12.35)\end{array}$ & $\begin{array}{c}4.25 \\
(6.62)\end{array}$ & $\begin{array}{c}3.10 \\
(6.58)\end{array}$ & $\begin{array}{c}3.29 \\
(10.44)\end{array}$ & $\begin{array}{c}2.65 \\
(10.49)\end{array}$ \\
\hline $\begin{array}{l}\text { When was the Diary } \\
\text { Completed? = 2, End } \\
\text { of diary day }\end{array}$ & 800 & & -182 & -169 & -363 & & 789 & & & 144 & & $812 *$ & 261 & 281 \\
\hline or alary aay & $(4.60)$ & $(4.52)$ & $\begin{array}{l}-1.82 \\
(2.47)\end{array}$ & $(2.47)$ & $(4.11)$ & $\begin{array}{l}-3.93 \\
(4.09)\end{array}$ & $(5.26)$ & $(5.26)$ & $(6.48)$ & $(6.53)$ & $(3.14)$ & $(3.15)$ & $(5.11)$ & $\begin{array}{l}2.81 \\
(5.12)\end{array}$ \\
\hline $\begin{array}{l}\text { When was the Diary } \\
\text { Completed? = 3, Day }\end{array}$ & & & & & & & & & & & & & & \\
\hline after diary day & $\begin{array}{c}5.03 \\
(5.24)\end{array}$ & $\begin{array}{c}6.28 \\
(5.16)\end{array}$ & $\begin{array}{l}-3.31 \\
(2.80)\end{array}$ & $\begin{array}{l}-3.17 \\
(2.83)\end{array}$ & $\begin{array}{l}1.28 \\
(4.69)\end{array}$ & $\begin{array}{c}1.24 \\
(4.71)\end{array}$ & $\begin{array}{l}7.48 \\
(6.70)\end{array}$ & $\begin{array}{l}8.02 \\
(6.79)\end{array}$ & $\begin{array}{l}1.77 \\
(7.91)\end{array}$ & $\begin{array}{l}0.30 \\
(7.94)\end{array}$ & $\begin{array}{l}-5.55 \\
(3.99)\end{array}$ & $\begin{array}{l}-5.41 \\
(3.99)\end{array}$ & $\begin{array}{l}-3.06 \\
(6.05)\end{array}$ & $\begin{array}{l}-2.55 \\
(6.09)\end{array}$ \\
\hline $\begin{array}{l}\text { When was the Diary } \\
\text { Completed? = 4, Later }\end{array}$ & $\begin{array}{l}9.16 \\
(6.60)\end{array}$ & $\begin{array}{c}9.78 \\
(6.65)\end{array}$ & $\begin{array}{l}-1.18 \\
(5.39)\end{array}$ & $\begin{array}{l}-0.86 \\
(5.41)\end{array}$ & $\begin{array}{c}1.49 \\
(5.71)\end{array}$ & $\begin{array}{c}1.34 \\
(5.78)\end{array}$ & $\begin{array}{c}0.31 \\
(7.45)\end{array}$ & $\begin{array}{c}0.51 \\
(7.37)\end{array}$ & $\begin{array}{c}-2.69 \\
(10.42)\end{array}$ & $\begin{array}{c}-3.61 \\
(10.41)\end{array}$ & $\begin{array}{c}-13.84^{*} \\
(5.91)\end{array}$ & $\begin{array}{c}-14.01 * \\
(5.90)\end{array}$ & $\begin{array}{c}6.23 \\
(8.63)\end{array}$ & $\begin{array}{c}6.46 \\
(8.59)\end{array}$ \\
\hline $\begin{array}{l}\text { When was the Diary } \\
\text { Completed? = 5, Don't }\end{array}$ & & & & & & & & & & & & & & \\
\hline know & $\begin{array}{c}20.88 \\
(18.44)\end{array}$ & $\begin{array}{c}19.45 \\
(18.50)\end{array}$ & $\begin{array}{l}-4.84 \\
(7.12)\end{array}$ & $\begin{array}{l}-3.77 \\
(7.01)\end{array}$ & $\begin{array}{l}-7.68 \\
(9.05)\end{array}$ & $\begin{array}{l}-6.91 \\
(9.26)\end{array}$ & $\begin{array}{c}-8.81 \\
(14.49)\end{array}$ & $\begin{array}{c}-8.41 \\
(14.32)\end{array}$ & $\begin{array}{c}10.06 \\
(12.99)\end{array}$ & $\begin{array}{c}9.62 \\
(13.09)\end{array}$ & $\begin{array}{l}-14.86 \\
(8.85)\end{array}$ & $\begin{array}{l}-16.27 \\
(8.62)\end{array}$ & $\begin{array}{l}-12.02 \\
(10.48)\end{array}$ & $\begin{array}{l}-12.05 \\
(10.55)\end{array}$ \\
\hline $\begin{array}{l}\text { Was the Diary } \\
\text { Completed with the } \\
\text { Study Child? = 2, } \\
\text { parent }\end{array}$ & -6.53 & -7.21 & -0.63 & -0.43 & -2.59 & & -8.13 & & & -7.21 & & & 4.98 & 5.05 \\
\hline & $(3.72)$ & (3.74) & $(1.76)$ & $(1.78)$ & $(3.22)$ & $(3.22)$ & $(4.15)$ & $(4.22)$ & $(5.48)$ & $(5.57)$ & (2.81) & $(2.82)$ & $(4.24)$ & $(4.22)$ \\
\hline $\begin{array}{l}\text { Was the Diary } \\
\text { Completed with the } \\
\text { Study Child? = 3, Don't }\end{array}$ & & & & & & & & & & & & & & \\
\hline know & $\begin{array}{l}-33.73 \\
(18.93)\end{array}$ & $\begin{array}{l}-32.13 \\
(18.91)\end{array}$ & $\begin{array}{c}2.71 \\
(7.31)\end{array}$ & $\begin{array}{l}1.75 \\
(7.17)\end{array}$ & $\begin{array}{l}15.79 \\
(9.71)\end{array}$ & $\begin{array}{l}15.27 \\
(9.80)\end{array}$ & $\begin{array}{c}3.46 \\
(14.65)\end{array}$ & $\begin{array}{c}3.61 \\
(14.51)\end{array}$ & $\begin{array}{l}-15.29 \\
(13.97)\end{array}$ & $\begin{array}{l}-15.04 \\
(14.03)\end{array}$ & $\begin{array}{l}11.71 \\
(9.22)\end{array}$ & $\begin{array}{l}12.80 \\
(9.02)\end{array}$ & $\begin{array}{c}23.57 \\
(11.52)\end{array}$ & $\begin{array}{c}23.55 \\
(11.64)\end{array}$ \\
\hline Mother's Marital & & & & & & & & & & & & & & \\
\hline Status $=1$, Married & & $\begin{array}{c}17.74 \\
(18.17)\end{array}$ & & $\begin{array}{c}30.39 \\
(21.76)\end{array}$ & & $\begin{array}{c}-6.74 \\
(16.97)\end{array}$ & & $\begin{array}{l}-25.93 \\
(20.14)\end{array}$ & & $\begin{array}{l}-20.69 \\
(27.49)\end{array}$ & & $\begin{array}{c}38.65 \\
(37.89)\end{array}$ & & $\begin{array}{c}47.51 \\
(31.17)\end{array}$ \\
\hline Father's Employment & & & & & & & & & & & & & & \\
\hline$=1$, Employed & & $22.34^{* *}$ & & -3.90 & & -3.59 & & 7.37 & & -10.23 & & 4.72 & & 2.95 \\
\hline $\begin{array}{l}\text { Father's Employment } \\
=2 \text {, omitted }\end{array}$ & & $(8.25)$ & & $(3.81)$ & & $(7.74)$ & & $(9.90)$ & & $(9.80)$ & & $(4.42)$ & & $(7.87)$ \\
\hline
\end{tabular}




\begin{tabular}{|c|c|c|c|c|c|c|c|c|c|c|c|c|c|c|}
\hline \multicolumn{15}{|l|}{ Mother's Employment } \\
\hline$=1$, Employed & & $(5.70)$ & & (2.50) & & $(5.03)$ & & $(6.50)$ & & (7.37) & & $(3.85)$ & & $(5.43)$ \\
\hline \multicolumn{15}{|l|}{ Number of People in } \\
\hline \multirow[t]{2}{*}{ Household } & & -7.98 & & 0.29 & & 6.55 & & 1.64 & & -0.18 & & -1.70 & & 1.52 \\
\hline & & (5.10) & & (3.16) & & $(4.30)$ & & $(4.58)$ & & (5.98) & & $(2.95)$ & & $(5.35)$ \\
\hline \multicolumn{15}{|l|}{ Father's Employment } \\
\hline \multirow[t]{2}{*}{$=2$, Not in household } & & $44.46 * * *$ & & 32.34 & & -9.64 & & -20.06 & & $-62.11 * * *$ & & 52.24 & & $64.48^{* *}$ \\
\hline & & $(10.08)$ & & $(21.60)$ & & $(12.80)$ & & $(17.86)$ & & $(14.16)$ & & (35.37) & & $(23.26)$ \\
\hline \multirow[t]{2}{*}{ Constant } & 52.49 & 89.05 & 11.60 & -24.98 & 60.88 & 22.84 & -7.62 & 8.81 & 46.78 & 74.42 & 82.41 & 58.92 & 51.01 & -9.58 \\
\hline & $(60.53)$ & (71.24) & $(30.93)$ & $(45.23)$ & (39.76) & $(48.59)$ & $(57.06)$ & $(69.77)$ & (71.65) & (81.70) & $(42.93)$ & $(60.16)$ & $(53.21)$ & $(68.74)$ \\
\hline Observations & 3,339 & 3,338 & 3,339 & 3,338 & 3,339 & 3,338 & 3,339 & 3,338 & 3,339 & 3,338 & 3,339 & 3,338 & 3,339 & 3,338 \\
\hline R-squared & 0.20 & 0.21 & 0.10 & 0.11 & 0.32 & 0.32 & 0.04 & 0.04 & 0.10 & 0.11 & 0.02 & 0.03 & 0.17 & 0.17 \\
\hline Number of ID & 2,563 & 2,563 & 2,563 & 2,563 & 2,563 & 2,563 & 2,563 & 2,563 & 2,563 & 2,563 & 2,563 & 2,563 & 2,563 & 2,563 \\
\hline
\end{tabular}

Notes: Clustered errors in parentheses. P-value alpha values of $0.01,0.05$, and 0.1 adjusted for multiple hypothesis testing using D/AP procedure according to the Bonferroni test with correlation $=0.15$ resulting in $\mathrm{p}$-value cut-offs: $* * * \mathrm{p}<0.0019,{ }^{* *} \mathrm{p}<0.0096, * \mathrm{p}<0.02$ 
Table A13 Full Table for fixed effects models (Boys)

\begin{tabular}{|c|c|c|c|c|c|c|c|c|c|c|c|c|c|c|}
\hline VARIABLES & $\begin{array}{c}(1) \\
\text { Sports } \\
\text { (min) }\end{array}$ & $\begin{array}{c}(2) \\
\text { Sports } \\
\text { (min) }\end{array}$ & $\begin{array}{c}(3) \\
\text { Reading } \\
(\min )\end{array}$ & $\begin{array}{c}(4) \\
\text { Reading } \\
(\text { min })\end{array}$ & $\begin{array}{c}(5) \\
\text { Homework } \\
\text { (min) }\end{array}$ & $\begin{array}{c}(6) \\
\text { Homework } \\
(\text { min })\end{array}$ & $\begin{array}{c}(7) \\
\text { Playing } \\
(\text { min) }\end{array}$ & $\begin{array}{c}(8) \\
\text { Playing } \\
(\text { min) }\end{array}$ & $\begin{array}{c}(9) \\
\text { Media } \\
\text { (min) }\end{array}$ & $\begin{array}{c}(10) \\
\text { Media } \\
(\text { min) }\end{array}$ & $\begin{array}{c}(11) \\
\text { Hobbies } \\
(\text { min) }\end{array}$ & $\begin{array}{c}(12) \\
\text { Hobbies } \\
(\text { min) }\end{array}$ & $\begin{array}{c}(13) \\
\text { Family } \\
\text { (min) }\end{array}$ & $\begin{array}{c}(14) \\
\text { Family } \\
(\text { min) }\end{array}$ \\
\hline Log income & $\begin{array}{l}-3.88 \\
(6.85)\end{array}$ & $\begin{array}{l}-2.09 \\
(7.34)\end{array}$ & $\begin{array}{l}-3.53 \\
(2.62)\end{array}$ & $\begin{array}{l}-3.91 \\
(2.56)\end{array}$ & $\begin{array}{l}-1.34 \\
(4.89)\end{array}$ & $\begin{array}{l}-2.36 \\
(4.91)\end{array}$ & $\begin{array}{l}-1.86 \\
(6.20)\end{array}$ & $\begin{array}{l}-2.07 \\
(6.31)\end{array}$ & $\begin{array}{l}-3.17 \\
(7.74)\end{array}$ & $\begin{array}{l}-2.57 \\
(8.23)\end{array}$ & $\begin{array}{l}-1.98 \\
(4.25)\end{array}$ & $\begin{array}{l}-0.78 \\
(4.28)\end{array}$ & $\begin{array}{c}5.52 \\
(6.18)\end{array}$ & $\begin{array}{c}1.45 \\
(6.56)\end{array}$ \\
\hline wave $=2$ & $\begin{array}{c}-25.92^{* * *} \\
(3.40)\end{array}$ & $\begin{array}{c}-25.74 * * * \\
(3.50)\end{array}$ & $\begin{array}{c}-12.26^{* * *} \\
(1.51)\end{array}$ & $\begin{array}{c}-12.62^{* * *} \\
(1.50)\end{array}$ & $\begin{array}{c}28.02^{* * *} \\
(2.45)\end{array}$ & $\begin{array}{c}26.56^{* * *} \\
(2.46)\end{array}$ & $\begin{array}{c}3.15 \\
(3.17)\end{array}$ & $\begin{array}{c}2.43 \\
(3.32)\end{array}$ & $\begin{array}{c}21.55^{* * *} \\
(4.19)\end{array}$ & $\begin{array}{c}23.46^{* * *} \\
(4.40)\end{array}$ & $\begin{array}{c}2.95 \\
(1.85)\end{array}$ & $\begin{array}{c}2.93 \\
(1.90)\end{array}$ & $\begin{array}{c}33.71^{* * *} \\
(3.16)\end{array}$ & $\begin{array}{c}34.12^{* * *} \\
(3.30)\end{array}$ \\
\hline DiaryDay $=3$, Wednesday & $\begin{array}{c}3.74 \\
(5.85)\end{array}$ & $\begin{array}{c}4.05 \\
(5.90)\end{array}$ & $\begin{array}{c}4.45 \\
(2.59)\end{array}$ & $\begin{array}{c}4.77 \\
(2.60)\end{array}$ & $\begin{array}{c}1.39 \\
(4.14)\end{array}$ & $\begin{array}{c}2.35 \\
(4.22)\end{array}$ & $\begin{array}{l}-9.11 \\
(5.31)\end{array}$ & $\begin{array}{l}-8.81 \\
(5.35)\end{array}$ & $\begin{array}{l}-4.62 \\
(7.11)\end{array}$ & $\begin{array}{l}-5.96 \\
(7.13)\end{array}$ & $\begin{array}{c}1.53 \\
(3.17)\end{array}$ & $\begin{array}{c}1.82 \\
(3.22)\end{array}$ & $\begin{array}{c}1.76 \\
(5.22)\end{array}$ & $\begin{array}{c}0.36 \\
(5.21)\end{array}$ \\
\hline DiaryDay $=4$, Thursday & $\begin{array}{c}4.56 \\
(6.19)\end{array}$ & $\begin{array}{c}4.64 \\
(6.30)\end{array}$ & $\begin{array}{c}2.19 \\
(2.48)\end{array}$ & $\begin{array}{c}2.35 \\
(2.48)\end{array}$ & $\begin{array}{c}3.12 \\
(4.16)\end{array}$ & $\begin{array}{c}3.01 \\
(4.17)\end{array}$ & $\begin{array}{l}-9.05 \\
(5.49)\end{array}$ & $\begin{array}{l}-9.25 \\
(5.52)\end{array}$ & $\begin{array}{l}-9.53 \\
(6.99)\end{array}$ & $\begin{array}{l}-9.34 \\
(7.08)\end{array}$ & $\begin{array}{c}5.61 \\
(3.33)\end{array}$ & $\begin{array}{c}5.76 \\
(3.36)\end{array}$ & $\begin{array}{c}2.23 \\
(6.22)\end{array}$ & $\begin{array}{c}1.59 \\
(6.24)\end{array}$ \\
\hline DiaryDay $=5$, Friday & $\begin{array}{c}8.42 \\
(6.49)\end{array}$ & $\begin{array}{c}8.58 \\
(6.57)\end{array}$ & $\begin{array}{l}-0.13 \\
(2.45)\end{array}$ & $\begin{array}{l}-0.05 \\
(2.43)\end{array}$ & $\begin{array}{c}-29.58^{* * *} \\
(4.13)\end{array}$ & $\begin{array}{c}-29.67^{* * *} \\
(4.12)\end{array}$ & $\begin{array}{c}15.50^{*} \\
(6.40)\end{array}$ & $\begin{array}{l}15.20^{*} \\
(6.38)\end{array}$ & $\begin{array}{l}13.44 \\
(7.79)\end{array}$ & $\begin{array}{l}13.60 \\
(7.80)\end{array}$ & $\begin{array}{l}-0.90 \\
(3.22)\end{array}$ & $\begin{array}{l}-0.87 \\
(3.23)\end{array}$ & $\begin{array}{c}22.10^{* * *} \\
(6.53)\end{array}$ & $\begin{array}{c}21.81^{* * *} \\
(6.52)\end{array}$ \\
\hline diarymonth $=2$, FEB & $\begin{array}{c}13.66 \\
(13.08)\end{array}$ & $\begin{array}{c}12.35 \\
(13.20)\end{array}$ & $\begin{array}{c}-13.18^{* *} \\
(4.67)\end{array}$ & $\begin{array}{c}-13.05^{* *} \\
(4.65)\end{array}$ & $\begin{array}{l}-10.42 \\
(7.70)\end{array}$ & $\begin{array}{l}-10.58 \\
(7.83)\end{array}$ & $\begin{array}{c}0.64 \\
(10.17)\end{array}$ & $\begin{array}{c}0.27 \\
(10.39)\end{array}$ & $\begin{array}{c}13.85 \\
(14.79)\end{array}$ & $\begin{array}{c}14.67 \\
(14.81)\end{array}$ & $\begin{array}{c}1.21 \\
(6.87)\end{array}$ & $\begin{array}{c}0.27 \\
(6.84)\end{array}$ & $\begin{array}{c}1.21 \\
(10.13)\end{array}$ & $\begin{array}{c}4.59 \\
(10.08)\end{array}$ \\
\hline diarymonth $=3, \mathrm{MAR}$ & $\begin{array}{c}4.57 \\
(10.21)\end{array}$ & $\begin{array}{c}4.15 \\
(10.17)\end{array}$ & $\begin{array}{l}-0.17 \\
(3.86)\end{array}$ & $\begin{array}{l}-0.07 \\
(3.86)\end{array}$ & $\begin{array}{l}-12.62 \\
(7.27)\end{array}$ & $\begin{array}{l}-12.27 \\
(7.24)\end{array}$ & $\begin{array}{l}29.61 * \\
(11.84)\end{array}$ & $\begin{array}{l}29.22 * \\
(11.85)\end{array}$ & $\begin{array}{c}5.32 \\
(11.95)\end{array}$ & $\begin{array}{c}5.22 \\
(11.96)\end{array}$ & $\begin{array}{l}-3.93 \\
(4.73)\end{array}$ & $\begin{array}{l}-4.57 \\
(4.85)\end{array}$ & $\begin{array}{l}-7.59 \\
(9.55)\end{array}$ & $\begin{array}{l}-5.73 \\
(9.73)\end{array}$ \\
\hline diarymonth $=4$, APR & $\begin{array}{c}38.18^{* * *} \\
(10.63)\end{array}$ & $\begin{array}{c}37.55^{* * *} \\
(10.64)\end{array}$ & $\begin{array}{l}-5.47 \\
(4.00)\end{array}$ & $\begin{array}{l}-5.73 \\
(4.01)\end{array}$ & $\begin{array}{l}-12.74 \\
(6.85)\end{array}$ & $\begin{array}{l}-12.86 \\
(6.83)\end{array}$ & $\begin{array}{l}-7.48 \\
(9.12)\end{array}$ & $\begin{array}{l}-7.07 \\
(9.07)\end{array}$ & $\begin{array}{c}5.42 \\
(10.15)\end{array}$ & $\begin{array}{c}5.59 \\
(10.16)\end{array}$ & $\begin{array}{l}-1.33 \\
(4.99)\end{array}$ & $\begin{array}{l}-1.72 \\
(5.12)\end{array}$ & $\begin{array}{l}-4.52 \\
(9.51)\end{array}$ & $\begin{array}{l}-2.70 \\
(9.46)\end{array}$ \\
\hline diarymonth $=5, \mathrm{MAY}$ & $\begin{array}{c}61.87^{* * *} \\
(18.83)\end{array}$ & $\begin{array}{c}61.03^{* * *} \\
(18.71)\end{array}$ & $\begin{array}{l}-4.41 \\
(5.71)\end{array}$ & $\begin{array}{l}-4.21 \\
(5.61)\end{array}$ & $\begin{array}{c}5.79 \\
(8.15)\end{array}$ & $\begin{array}{c}5.98 \\
(8.09)\end{array}$ & $\begin{array}{c}17.00 \\
(13.49)\end{array}$ & $\begin{array}{c}16.51 \\
(13.48)\end{array}$ & $\begin{array}{l}-22.99 \\
(21.01)\end{array}$ & $\begin{array}{l}-22.70 \\
(21.21)\end{array}$ & $\begin{array}{c}2.65 \\
(7.51)\end{array}$ & $\begin{array}{c}1.84 \\
(7.62)\end{array}$ & $\begin{array}{c}-5.47 \\
(12.76)\end{array}$ & $\begin{array}{c}-2.94 \\
(12.79)\end{array}$ \\
\hline diarymonth $=6$, JUN & $\begin{array}{c}34.68 \\
(34.04)\end{array}$ & $\begin{array}{c}33.70 \\
(33.95)\end{array}$ & $\begin{array}{c}11.59 \\
(13.59)\end{array}$ & $\begin{array}{c}11.09 \\
(13.47)\end{array}$ & $\begin{array}{l}-10.25 \\
(15.58)\end{array}$ & $\begin{array}{l}-12.44 \\
(15.27)\end{array}$ & $\begin{array}{l}-42.09 \\
(23.79)\end{array}$ & $\begin{array}{l}-42.04 \\
(24.26)\end{array}$ & $\begin{array}{c}-2.15 \\
(35.87)\end{array}$ & $\begin{array}{c}0.73 \\
(35.66)\end{array}$ & $\begin{array}{c}2.20 \\
(17.94)\end{array}$ & $\begin{array}{c}2.15 \\
(18.13)\end{array}$ & $\begin{array}{c}43.11^{* *} \\
(14.03) \\
-\end{array}$ & $\begin{array}{l}44.72^{* *} \\
(14.79)\end{array}$ \\
\hline diarymonth $=7$, JUL & $\begin{array}{c}-38.54^{* * *} \\
(6.19)\end{array}$ & $\begin{array}{c}-36.30^{* * *} \\
(7.99)\end{array}$ & $\begin{array}{c}2.04 \\
(2.54)\end{array}$ & $\begin{array}{c}3.05 \\
(3.38)\end{array}$ & $\begin{array}{c}-26.44^{* * *} \\
(4.27)\end{array}$ & $\begin{array}{c}-24.42^{* * *} \\
(5.59)\end{array}$ & $\begin{array}{c}27.78^{* * *} \\
(5.76)\end{array}$ & $\begin{array}{c}22.60^{* *} \\
(7.93)\end{array}$ & $\begin{array}{c}-95.31 * * * \\
(7.81)\end{array}$ & $\begin{array}{c}-96.42^{* * *} \\
(10.36)\end{array}$ & $\begin{array}{l}-1.07 \\
(3.87)\end{array}$ & $\begin{array}{l}-3.16 \\
(5.05)\end{array}$ & $\begin{array}{c}31.48^{* * *} \\
(5.65)\end{array}$ & $\begin{array}{c}-29.12^{* * *} \\
(7.74)\end{array}$ \\
\hline diarymonth $=8$, AUG & $\begin{array}{c}25.39 \\
(18.83)\end{array}$ & $\begin{array}{c}24.77 \\
(18.72)\end{array}$ & $\begin{array}{c}3.39 \\
(10.66)\end{array}$ & $\begin{array}{c}3.19 \\
(10.56)\end{array}$ & $\begin{array}{l}-28.32 \\
(13.74)\end{array}$ & $\begin{array}{l}-29.19 \\
(13.92)\end{array}$ & $\begin{array}{c}8.33 \\
(26.52)\end{array}$ & $\begin{array}{c}7.54 \\
(26.50)\end{array}$ & $\begin{array}{c}4.00 \\
(15.29)\end{array}$ & $\begin{array}{c}5.55 \\
(15.05)\end{array}$ & $\begin{array}{c}0.69 \\
(12.98)\end{array}$ & $\begin{array}{c}-0.09 \\
(12.61)\end{array}$ & $\begin{array}{c}25.42 \\
(16.97)\end{array}$ & $\begin{array}{c}28.22 \\
(15.28)\end{array}$ \\
\hline diarymonth $=9$, SEP & $32.32 * * *$ & $31.27^{* * *}$ & -3.67 & -3.57 & -5.13 & -4.87 & -5.09 & -4.81 & -5.57 & -5.59 & -4.66 & -5.21 & -5.37 & -3.20 \\
\hline
\end{tabular}




\begin{tabular}{|c|c|c|c|c|c|c|c|c|c|c|c|c|c|c|}
\hline & $(8.62)$ & $(8.57)$ & (3.53) & (3.49) & $(6.00)$ & $(5.98)$ & $(8.25)$ & $(8.21)$ & $(10.05)$ & $(9.98)$ & $(4.84)$ & $(4.95)$ & $(7.53)$ & $(7.44)$ \\
\hline \multirow[t]{2}{*}{ diarymonth $=10, \mathrm{OCT}$} & $19.17^{* *}$ & $18.53^{*}$ & -1.21 & -1.28 & -7.23 & -7.70 & -0.94 & -1.14 & 0.71 & 1.56 & -0.36 & -0.78 & -8.25 & -6.55 \\
\hline & $(7.36)$ & (7.39) & (3.42) & (3.40) & $(5.34)$ & $(5.31)$ & $(7.18)$ & $(7.15)$ & $(8.81)$ & $(8.74)$ & (4.33) & $(4.37)$ & $(7.22)$ & $(7.15)$ \\
\hline \multirow[t]{2}{*}{ diarymonth $=11, \mathrm{NOV}$} & 13.14 & 12.43 & -7.56 & -7.63 & -7.57 & -7.83 & 1.48 & 1.47 & -0.78 & -0.22 & -0.03 & -0.47 & -2.89 & -1.09 \\
\hline & (7.39) & $(7.37)$ & (3.52) & (3.46) & $(5.62)$ & $(5.58)$ & $(7.51)$ & $(7.55)$ & $(8.60)$ & $(8.60)$ & $(4.63)$ & $(4.71)$ & $(7.26)$ & $(7.09)$ \\
\hline \multirow[t]{2}{*}{ diarymonth $=12, \mathrm{DEC}$} & 4.47 & 4.00 & -3.05 & -2.97 & 0.94 & 0.74 & -4.38 & -4.70 & 8.34 & 8.88 & -0.40 & -0.76 & 0.50 & 1.74 \\
\hline & (7.26) & (7.28) & (3.47) & (3.45) & $(5.72)$ & $(5.73)$ & $(7.33)$ & $(7.25)$ & $(9.04)$ & (8.95) & $(4.54)$ & (4.58) & $(7.37)$ & $(7.27)$ \\
\hline \multirow{3}{*}{$\begin{array}{l}\text { When was the Diary } \\
\text { Completed? = 2, End of } \\
\text { diary day }\end{array}$} & & & & & & & & & & & & & & \\
\hline & 1.52 & 1.10 & -1.08 & -0.99 & -2.05 & -1.89 & -9.31 & -8.86 & -8.90 & -9.10 & 2.21 & 2.26 & -4.01 & -3.92 \\
\hline & $(5.87)$ & $(5.85)$ & (2.35) & (2.33) & (3.64) & (3.59) & $(5.22)$ & $(5.20)$ & $(6.68)$ & $(6.66)$ & (3.08) & (3.07) & $(4.87)$ & $(4.88)$ \\
\hline \multirow{3}{*}{$\begin{array}{l}\text { When was the Diary } \\
\text { Completed? = 3, Day after } \\
\text { diary day }\end{array}$} & & & & & & & & & & & & & & \\
\hline & 2.81 & 3.35 & -2.76 & -2.77 & 3.11 & 2.79 & -0.19 & -0.71 & -6.66 & -6.26 & -2.37 & -2.21 & -7.66 & -8.47 \\
\hline & $(5.92)$ & $(5.88)$ & $(2.82)$ & $(2.85)$ & $(4.03)$ & $(4.00)$ & $(5.70)$ & $(5.71)$ & $(7.10)$ & $(7.06)$ & (3.49) & (3.48) & $(5.95)$ & $(5.93)$ \\
\hline \multicolumn{15}{|l|}{ When was the Diary } \\
\hline \multirow[t]{2}{*}{ Completed $=4$, Later } & 3.07 & 3.46 & -6.53 & -6.81 & -2.80 & -3.25 & -13.49 & -13.82 & 7.20 & 7.69 & -1.90 & -1.95 & -6.30 & -6.14 \\
\hline & (9.12) & (9.18) & (3.36) & (3.36) & $(4.74)$ & $(4.74)$ & $(7.89)$ & $(7.80)$ & $(10.23)$ & $(10.27)$ & (4.48) & (4.45) & $(8.75)$ & $(8.58)$ \\
\hline \multicolumn{15}{|l|}{$\begin{array}{l}\text { When was the Diary } \\
\text { Completed? = 5, Don't }\end{array}$} \\
\hline \multirow[t]{2}{*}{ know } & 20.63 & 21.35 & 6.23 & 5.99 & -10.55 & -10.56 & 20.88 & 19.82 & -2.66 & -2.53 & -4.03 & -4.56 & -3.69 & -2.62 \\
\hline & $(12.74)$ & $(12.75)$ & $(5.70)$ & $(5.62)$ & (10.98) & $(10.86)$ & (13.38) & (13.19) & $(16.76)$ & $(17.21)$ & $(5.38)$ & $(5.43)$ & (16.17) & (15.67) \\
\hline \multirow{3}{*}{$\begin{array}{l}\text { Was the Diary Completed } \\
\text { with the Study Child? = 2, } \\
\text { parent }\end{array}$} & & & & & & & & & & & & & & \\
\hline & 2.36 & 2.22 & 1.62 & 1.65 & 4.80 & 5.04 & -3.26 & -2.81 & -3.15 & -3.56 & 0.59 & 0.72 & 4.96 & 4.68 \\
\hline & $(4.05)$ & (4.05) & (1.62) & (1.61) & $(2.88)$ & $(2.87)$ & $(4.24)$ & $(4.23)$ & $(4.78)$ & $(4.75)$ & $(2.27)$ & $(2.28)$ & $(3.82)$ & $(3.84)$ \\
\hline \multicolumn{15}{|l|}{$\begin{array}{l}\text { Was the Diary Completed } \\
\text { with the Study Child? = }\end{array}$} \\
\hline \multirow[t]{2}{*}{ Don't know } & -7.02 & -8.00 & -7.21 & -6.74 & 11.93 & 12.30 & -20.46 & -18.99 & -2.48 & -3.09 & 4.97 & 5.71 & 2.33 & 0.67 \\
\hline & $(12.64)$ & $(12.62)$ & (5.63) & $(5.50)$ & $(10.64)$ & (10.53) & (14.54) & (14.27) & (16.59) & (16.99) & $(5.61)$ & (5.73) & (17.01) & (16.54) \\
\hline \multirow{3}{*}{$\begin{array}{l}\text { Mother's Marital Status = } \\
1, \text { Married }\end{array}$} & & & & & & & & & & & & & & \\
\hline & & -12.78 & & 10.78 & & 8.00 & & 0.87 & & -4.22 & & 0.20 & & -2.70 \\
\hline & & $(21.86)$ & & (6.65) & & $(9.90)$ & & $(17.16)$ & & $(20.85)$ & & (7.32) & & $(17.41)$ \\
\hline \multicolumn{15}{|l|}{ Father's Employment $=1$, } \\
\hline \multirow[t]{2}{*}{ Employed } & & -6.04 & & -3.42 & & -9.64 & & -3.88 & & 14.77 & & -5.85 & & $24.41^{* *}$ \\
\hline & & $(7.98)$ & & (3.95) & & (6.39) & & $(9.75)$ & & (11.43) & & (4.95) & & $(9.21)$ \\
\hline $\begin{array}{l}\text { Father's Employment = 2, } \\
\text { omitted }\end{array}$ & & - & & - & & - & & - & & - & & - & & - \\
\hline Mother's Employment $=1$, & & & & & & & & & & & & & & \\
\hline Employed & & -10.09 & & 2.66 & & 9.53 & & 11.29 & & -11.75 & & -0.93 & & 7.04 \\
\hline
\end{tabular}




\begin{tabular}{|c|c|c|c|c|c|c|c|c|c|c|c|c|c|c|}
\hline \multirow{2}{*}{\multicolumn{2}{|c|}{ Number of People in }} & $(6.98)$ & & (3.53) & & $(4.31)$ & & $(6.46)$ & & $(7.53)$ & & $(4.36)$ & & $(6.18)$ \\
\hline & & & & & & & & & & & & & & \\
\hline \multicolumn{2}{|l|}{ Household } & -1.62 & & -1.37 & & -3.68 & & 3.56 & & 3.44 & & 1.83 & & -1.72 \\
\hline & & $(4.99)$ & & $(2.32)$ & & $(3.55)$ & & $(5.51)$ & & $(6.36)$ & & (3.18) & & $(5.08)$ \\
\hline \multicolumn{15}{|c|}{$\begin{array}{l}\text { Father's Employment = 2, } \\
\text { Not in household }\end{array}$} \\
\hline \multirow[t]{2}{*}{ Constant } & 81.04 & 94.43 & 57.39 & 58.94 & 65.99 & 88.56 & 56.08 & 37.00 & 119.88 & 95.91 & 31.11 & 16.15 & -39.62 & -14.64 \\
\hline & (67.97) & (78.49) & (25.91) & (28.86) & (49.29) & (53.79) & $(60.25)$ & (68.42) & (76.75) & $(92.80)$ & $(41.84)$ & $(46.03)$ & $(61.20)$ & (72.70) \\
\hline Observations & 3,340 & 3,340 & 3,340 & 3,340 & 3,340 & 3,340 & 3,340 & 3,340 & 3,340 & 3,340 & 3,340 & 3,340 & 3,340 & 3,340 \\
\hline R-squared & 0.19 & 0.19 & 0.15 & 0.15 & 0.30 & 0.31 & 0.07 & 0.08 & 0.09 & 0.10 & 0.02 & 0.02 & 0.19 & 0.20 \\
\hline Number of ID & 2,546 & 2,546 & 2,546 & 2,546 & 2,546 & 2,546 & 2,546 & 2,546 & 2,546 & 2,546 & 2,546 & 2,546 & 2,546 & 2,546 \\
\hline
\end{tabular}

Notes: Clustered errors in parentheses. P-value alpha values of $0.01,0.05$, and 0.1 adjusted for multiple hypothesis testing using D/AP procedure according to the Bonferroni test with

correlation $=0.15$ resulting in $\mathrm{p}$-value cut-offs: $* * * \mathrm{p}<0.0019, * * \mathrm{p}<0.0096, * \mathrm{p}<0.02$ 
Table A14 Results for time spent in activities for girls and boys using both weekday and weekend data

\begin{tabular}{|c|c|c|c|c|c|c|c|c|c|c|c|c|c|c|}
\hline \multirow[b]{2}{*}{ Variables } & \multicolumn{7}{|c|}{ Girls Pooled OLS } & \multicolumn{7}{|c|}{ Boys Pooled OLS } \\
\hline & Sport (Min) & $\begin{array}{l}\text { Reading } \\
\text { (Min) }\end{array}$ & $\begin{array}{l}\text { Homewor } \\
\text { k (Min) }\end{array}$ & $\begin{array}{l}\text { Playing } \\
\text { (Min) }\end{array}$ & $\begin{array}{l}\text { Media } \\
\text { (Min) }\end{array}$ & $\begin{array}{l}\text { Hobbies } \\
\text { (Min) }\end{array}$ & $\begin{array}{l}\text { Family } \\
\text { (Min) }\end{array}$ & $\begin{array}{l}\text { Sport } \\
\text { (Min) }\end{array}$ & $\begin{array}{l}\text { Reading } \\
\text { (Min) }\end{array}$ & $\begin{array}{l}\text { Homework } \\
\text { (Min) }\end{array}$ & $\begin{array}{l}\text { Playing } \\
\text { (Min) }\end{array}$ & $\begin{array}{l}\text { Media } \\
\text { (Min) }\end{array}$ & $\begin{array}{l}\text { Hobbies } \\
\text { (Min) }\end{array}$ & $\begin{array}{l}\text { Family } \\
\text { (Min) }\end{array}$ \\
\hline \multicolumn{15}{|l|}{ Mother's Education } \\
\hline \multirow[t]{2}{*}{ Secondary } & 1.08 & 0.10 & -1.85 & 5.47 & -3.43 & 2.66 & 3.49 & 2.51 & $4.99 * * *$ & -4.61 & 5.61 & -6.02 & -0.86 & 6.98 \\
\hline & $(4.67)$ & (2.35) & (1.89) & $(4.45)$ & $(5.33)$ & (2.63) & $(4.98)$ & $(5.80)$ & (1.57) & $(2.00)$ & $(4.11)$ & $(5.28)$ & $(2.60)$ & $(5.51)$ \\
\hline \multirow[t]{2}{*}{ More than Secondary } & -0.16 & $5.78^{*}$ & -3.37 & 7.64 & -7.48 & $6.70^{* *}$ & 8.58 & 5.52 & $12.11 * * *$ & $-5.39 * *$ & 8.16 & -7.23 & 3.22 & 2.94 \\
\hline & (4.35) & (2.24) & $(1.84)$ & $(4.30)$ & $(4.80)$ & $(2.44)$ & -5.19 & $(5.40)$ & $(1.71)$ & (1.88) & (3.71) & $(5.25)$ & $(2.71)$ & $(5.54)$ \\
\hline \multirow[t]{2}{*}{ Wave $=2$} & $-36.16^{* * *}$ & $-9.65 * * *$ & $27.20^{* * *}$ & $22.43^{*}$ & $51.54^{* * *}$ & 13.51 & $30.45^{* * *}$ & $-44.54^{* * *}$ & $-6.03^{* * *}$ & $14.46^{* * *}$ & $40.46^{* * *}$ & $24.37^{*}$ & 14.11 & $47.30^{* * *}$ \\
\hline & (6.32) & (3.06) & $(5.34)$ & $(8.86)$ & (13.30) & $(9.20)$ & (9.11) & $(6.83)$ & (1.77) & $(4.17)$ & $(10.03)$ & $(9.42)$ & $(6.45)$ & $(10.20)$ \\
\hline \multirow[t]{2}{*}{ Secondary * Wave 2} & 3.20 & 0.85 & 5.15 & -15.05 & -12.90 & -13.80 & 4.11 & 17.22 & -3.99 & $13.32^{* *}$ & $-25.87^{*}$ & 15.49 & -4.15 & -25.20 \\
\hline & (7.46) & (3.32) & $(5.81)$ & $(10.05)$ & (14.13) & (9.94) & $(10.20)$ & (9.86) & (2.14) & $(4.86)$ & (11.07) & $(10.96)$ & (6.73) & (11.02) \\
\hline \multirow[t]{2}{*}{$\begin{array}{l}\text { More than Secondary * } \\
\text { Wave } 2\end{array}$} & 15.13 & 0.25 & 5.42 & -23.29 & -14.23 & -11.34 & -9.37 & 11.42 & $-6.04 * *$ & $13.23^{* *}$ & $-36.60 * * *$ & 12.77 & -10.48 & $-24.05 * *$ \\
\hline & (7.78) & $(3.40)$ & (5.64) & (10.06) & (13.84) & $(10.72)$ & (10.36) & (7.87) & (2.31) & $(4.55)$ & (10.44) & $(10.47)$ & $(6.66)$ & $(10.90)$ \\
\hline Diary Controls & $Y$ & $Y$ & $Y$ & $Y$ & $Y$ & $Y$ & $Y$ & $Y$ & $Y$ & $Y$ & $Y$ & $Y$ & $Y$ & $Y$ \\
\hline \multirow[t]{2}{*}{ Constant } & $30.84^{* * *}$ & $24.45^{* * *}$ & $52.17 * * *$ & $40.54^{* * *}$ & $92.40^{* * *}$ & $16.06^{* * *}$ & 13.22 & $48.90^{* * *}$ & $14.54^{* * *}$ & $50.21^{* * *}$ & $22.40^{* * *}$ & $92.73^{* * *}$ & $\underset{*}{11.11^{* *}}$ & 14.93 \\
\hline & $(5.81)$ & (3.61) & $(4.15)$ & (7.49) & (8.18) & $(4.92)$ & (6.87) & (7.33) & (2.21) & (3.67) & (5.55) & (7.91) & (3.44) & (7.90) \\
\hline Observations & 5,593 & 5,593 & 5,593 & 5,593 & 5,593 & 5,593 & 5,593 & 5,574 & 5,574 & 5,574 & 5,574 & 5,574 & 5,574 & 5,574 \\
\hline R-Squared & 0.15 & 0.04 & 0.26 & 0.08 & 0.14 & 0.05 & 0.18 & 0.14 & 0.06 & 0.23 & 0.08 & 0.18 & 0.04 & 0.16 \\
\hline
\end{tabular}

Notes: Omitted category is children in households whose mothers have less than secondary education. Clustered errors in parentheses. P-value alpha values of $0.01,0.05$, and 0.1 adjusted

for multiple hypothesis testing using D/AP procedure according to the Bonferroni test with correlation $=0.15$ resulting in $p$-value cut-offs: ${ }^{* * *} p<0.0019, * * p<0.0096,{ }^{*} p<0.02$ 


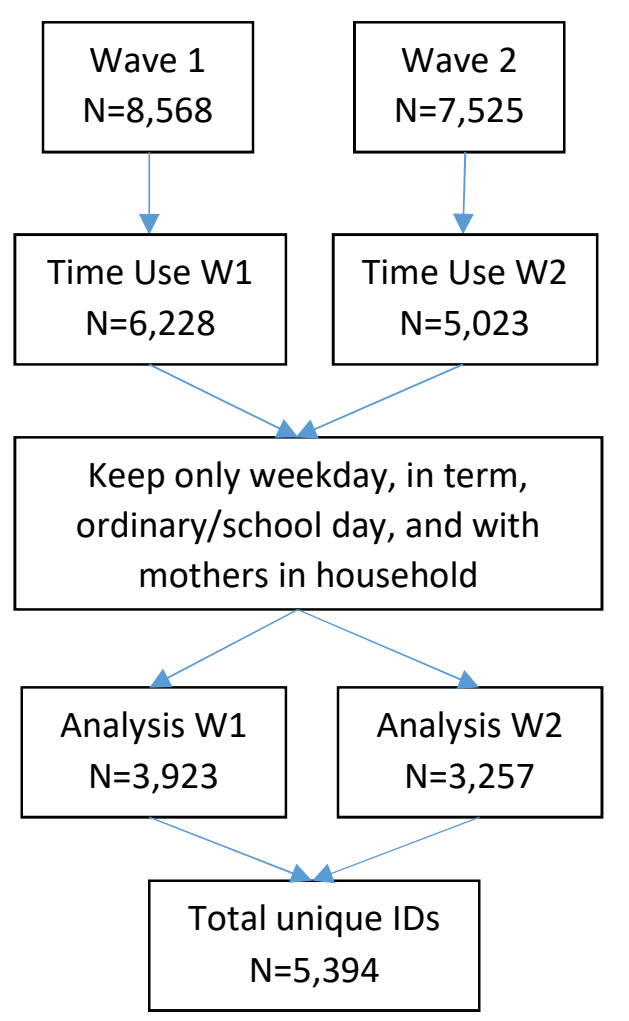

Fig. A1 Construction of Analysis Data 


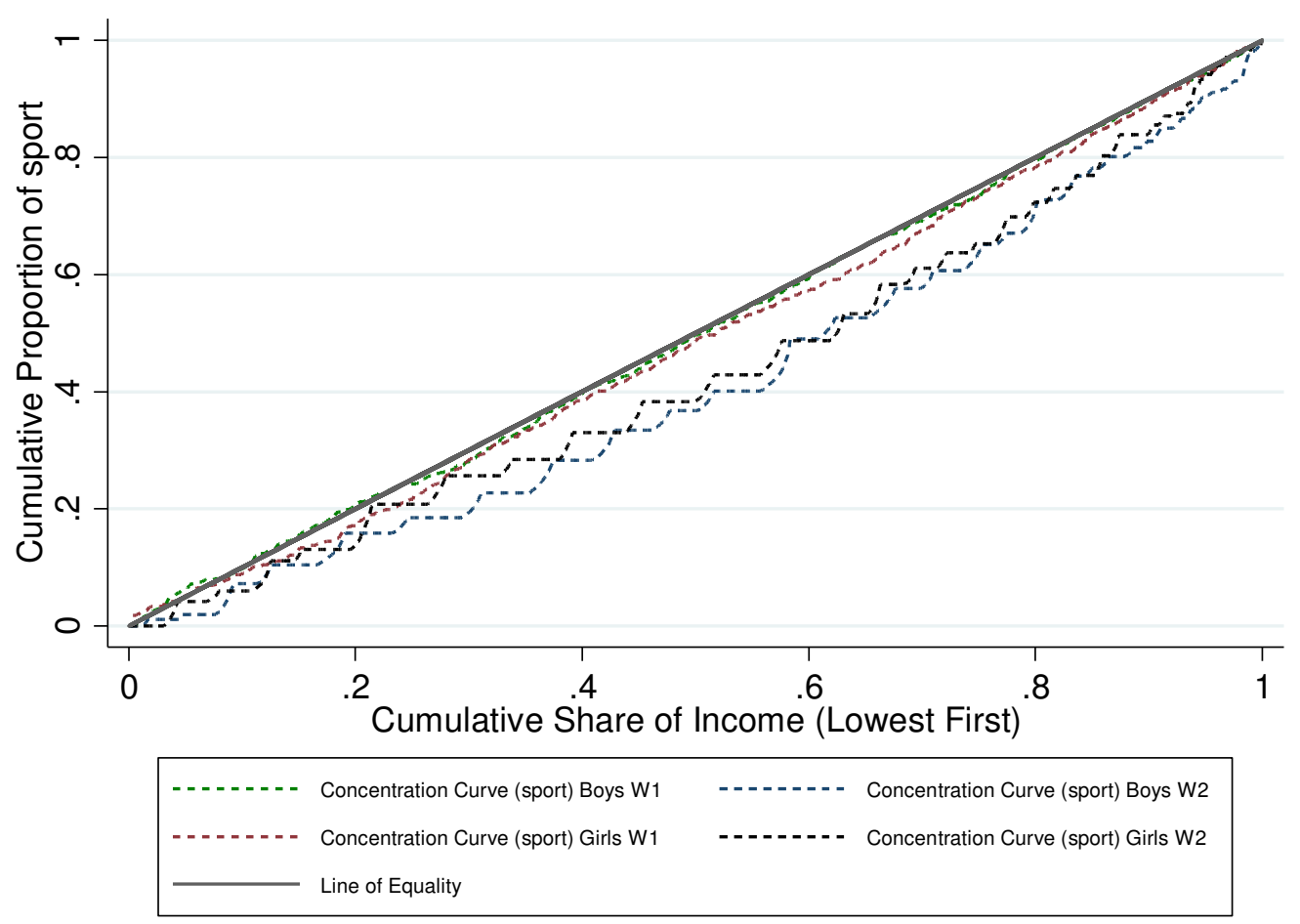

Figure A2. Concentration curves for sports time for boys and girls at waves 1 and 2

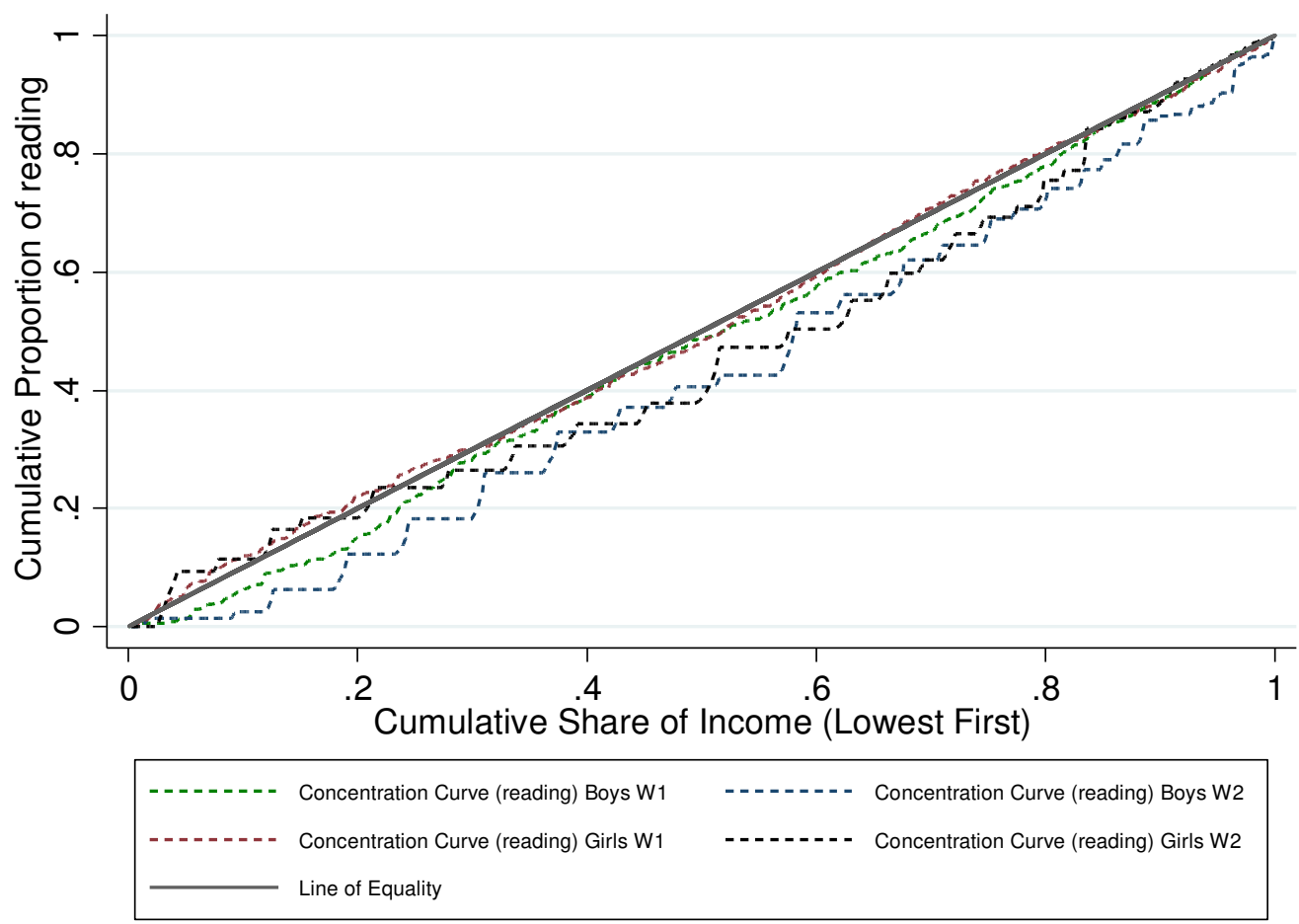

Figure A3. Concentration curves for reading time for boys and girls at waves 1 and 2 


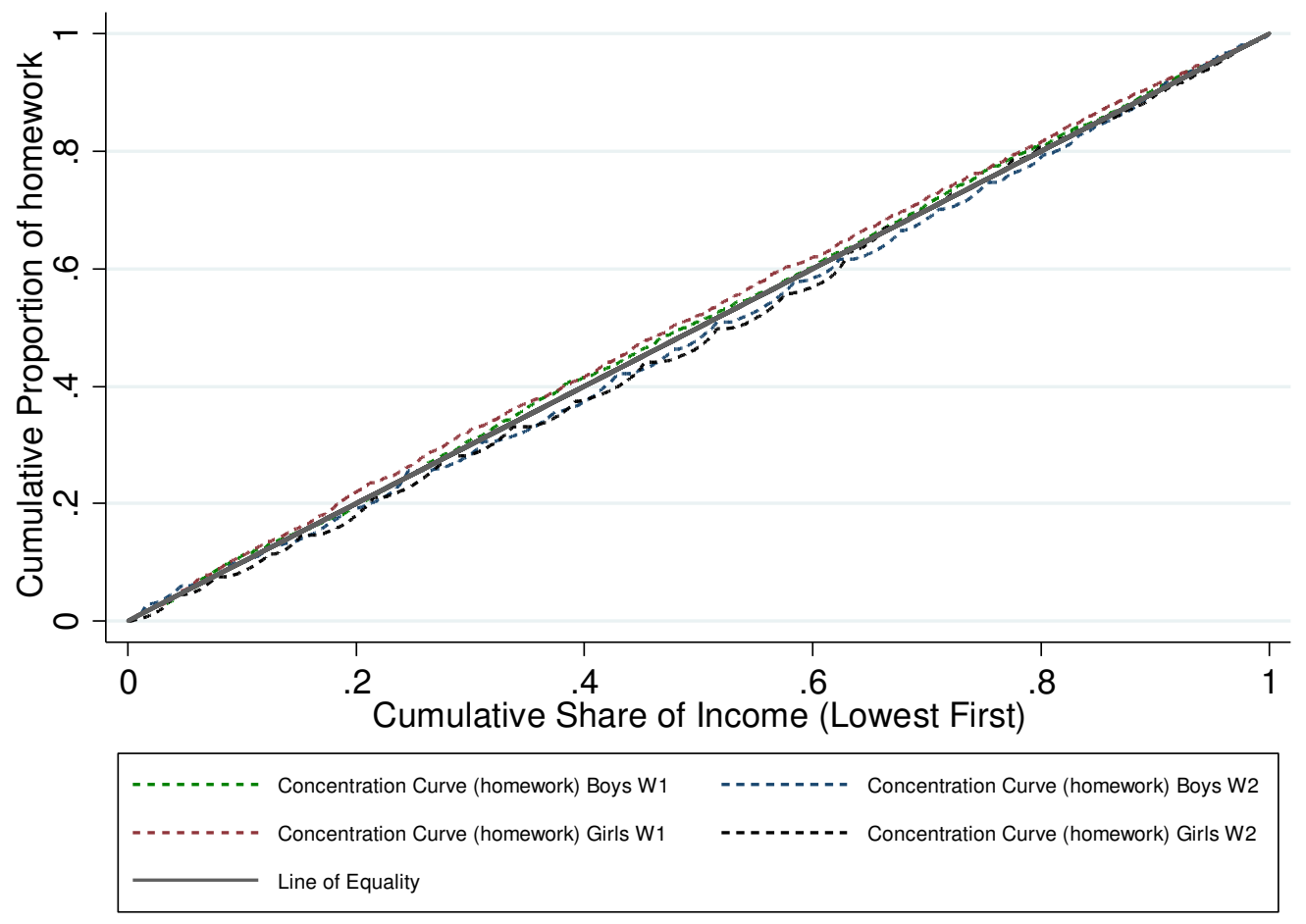

Figure A4. Concentration curves for homework time for boys and girls at waves 1 and 2

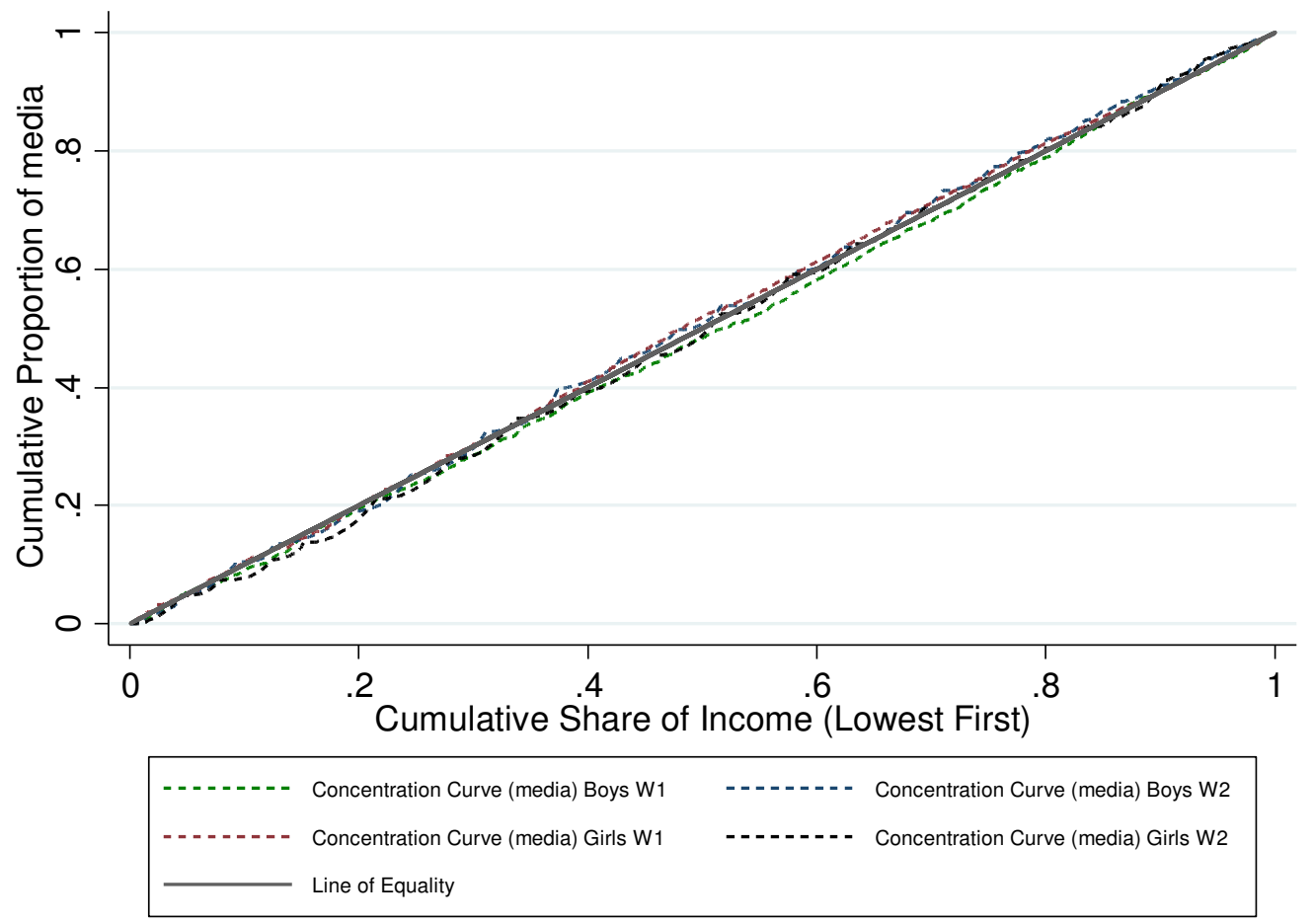

Figure A5. Concentration curves for media time for boys and girls at waves 1 and 2 


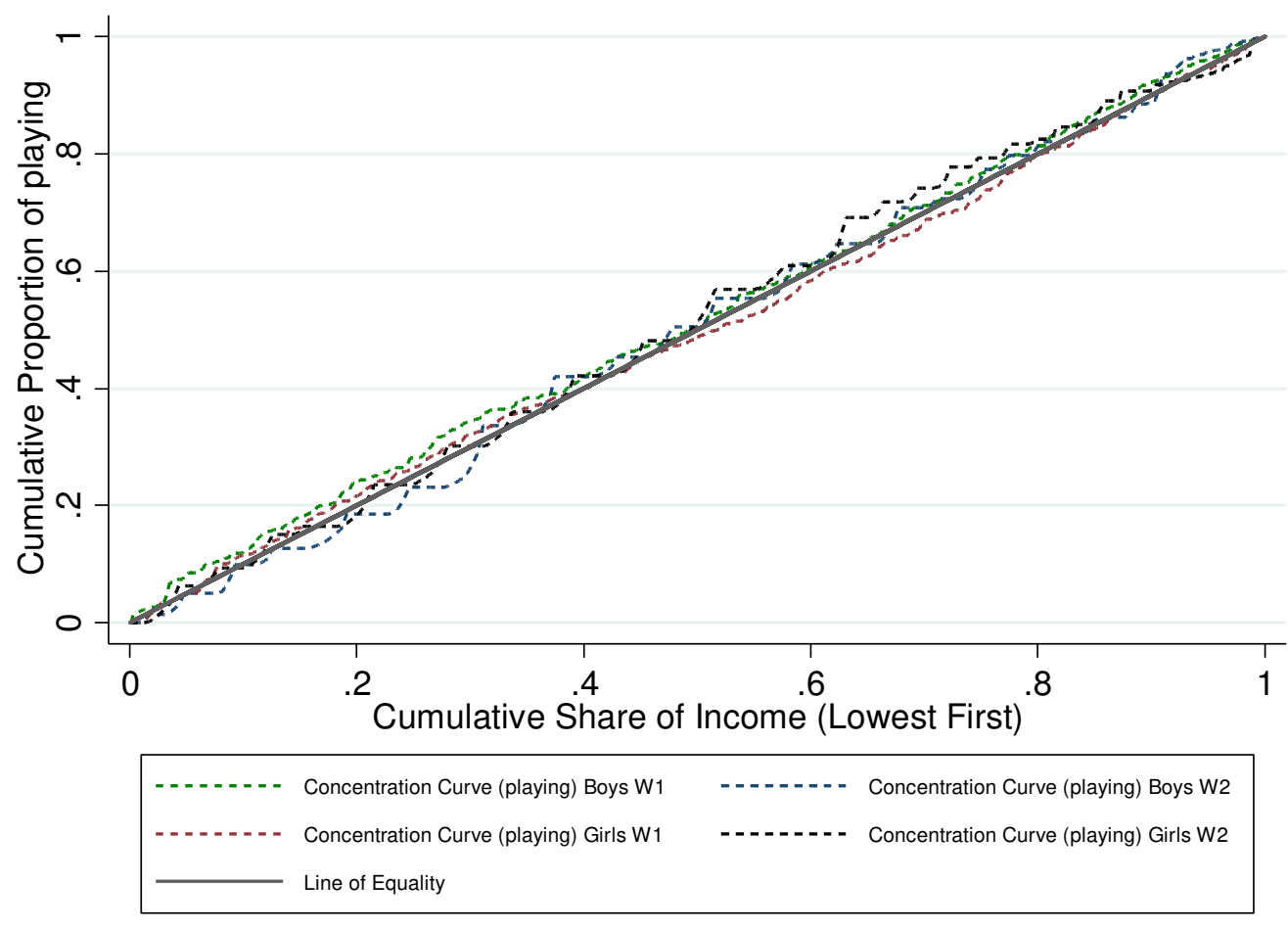

Figure A6. Concentration curves for playing time for boys and girls at waves 1 and 2

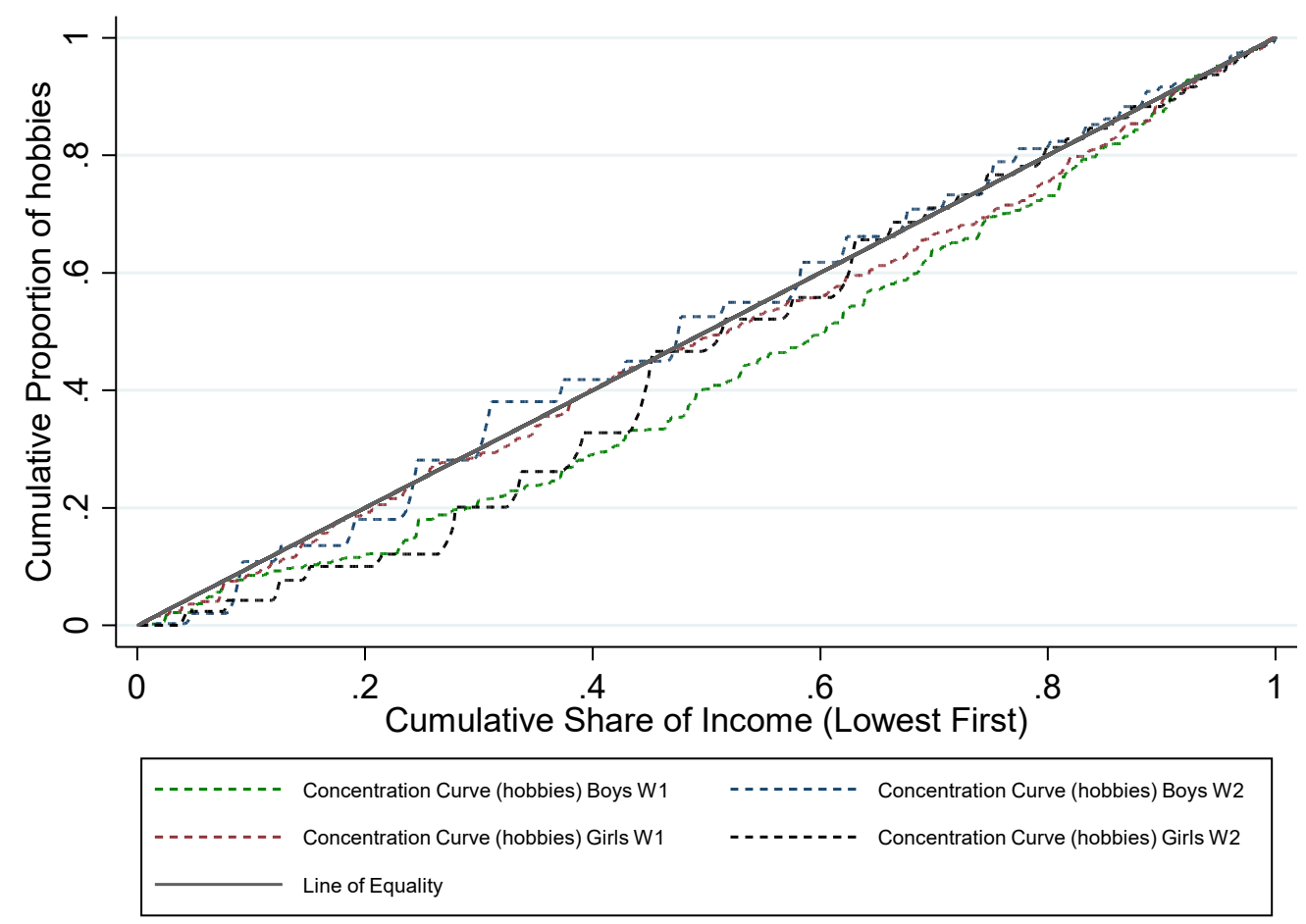

Figure A7. Concentration curves for hobbies time for boys and girls at waves 1 and 2 


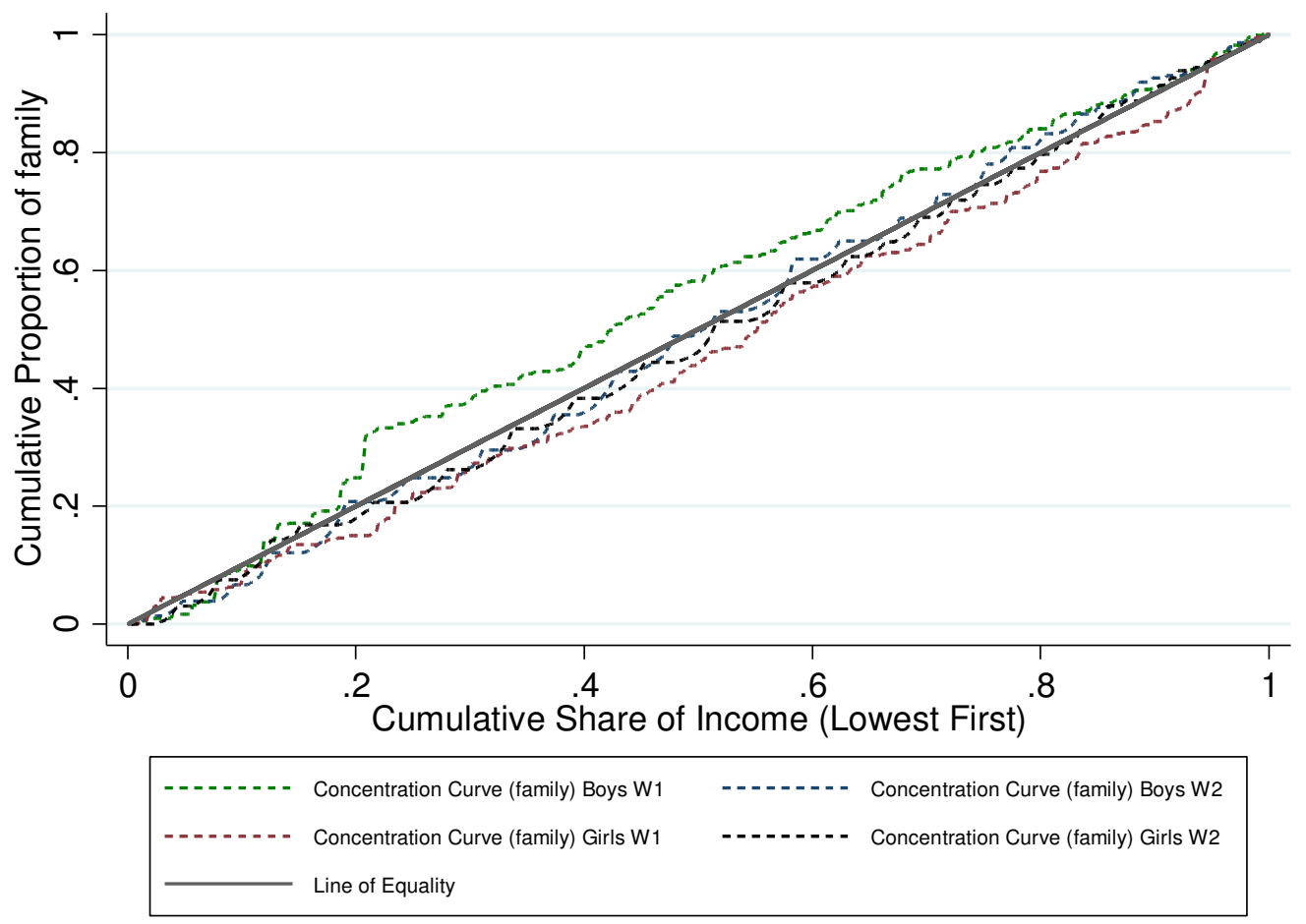

Figure A8. Concentration curves for family time for boys and girls at waves 1 and 2 


\section{Section A1. Theoretical Framework}

In this paper, we are concerned not only with describing SES differences in parental investments as measured by time use, but also establishing the extent to which these gaps widen over time. Here we present an outline of a theoretical framework that illustrates two key concepts, self-complementarity and cross-productivity, which provide a potential explanation for changes in average investments across families as children age. While these have specific economic interpretations, we argue below that these can be viewed as manifestations of concepts discussed in other literatures in, for example, psychology and sociology. This framework also provides insights into why time investments may vary across different types of activities.

Following Becker and Tomes (1986) and Cunha and Heckman (2007), consider a three period model where adult earnings at time $t,\left(Y_{t}\right)$, are a function of adult human capital $\left(H_{t}\right)$ :

$$
Y_{t}=f_{t}\left(H_{t}\right)
$$

Adult human capital depends on a vector of determinants formed in the childhood periods, as well as initial endowments $(\gamma)$. For the purposes of this analysis we focus on two childhood time periods $(t-$ $1, t-2)$ and parental investments as measured by time inputs $\left(\right.$ Time $_{t-1}$, Time $\left._{t-2}\right)$, while the vector $X$ includes family background and societal factors that are expected to influence skill acquisition, such as public expenditure and macroeconomic environment, some of which will be time invariant, others will vary as children age (for example family income).

$$
H_{t}=\theta_{t-1}=f_{t-1}\left(\theta_{\left.t-2, \text { Time }_{t-1}, \gamma, X\right)}\right.
$$

In Eq. (2), $H_{t}$ is given by the stock of skills in the second childhood period $\left(\theta_{t-1}\right)$, which depends on initial endowments, time inputs in both childhood periods, the stock of skills in the first childhood period $\left(\theta_{t-2,}=f_{t-2}\left(\right.\right.$ Time $\left.\left._{t-2}, \gamma, X\right)\right)$, and other determinants in $X$, with $\frac{\partial H_{t}}{\partial \theta_{t-2}}, \frac{\partial H_{t}}{\partial \text { Time }_{t-1}}$, and $\frac{\partial H_{t}}{\partial \gamma}$ all $>$ 0 . Time $_{t-1}$, Time $_{t-2}$ are vectors of time inputs associated with activities $j_{1}, j_{2}, \ldots j_{J}$.

As observations from psychological and biological literatures support the hypothesis that there are sensitive periods for development, we expect a non-linear relationship between the stock of skills in the first and second childhood periods. In particular, having greater skills in the first childhood period is likely to contribute to a higher stock of skills in the second childhood period:

$$
\frac{\partial f_{t-1}\left(\theta_{\left.t-2, \text { Time }_{t-1}, \text { Time }_{t-2}, \gamma, X\right)}\right.}{\partial \theta_{t-2}}>0
$$

In the economics literature this is referred to as self-productivity, and can arise, when, for example, better psycho-social skills acquired in preschool enable children to improve their academic ability in primary school. These effects can apply both within the same skill sets and across abilities.

The literature on sensitive periods also supports there being greater returns to earlier investments (Doyle et al. 2009), and this model can also incorporate this observation:

$$
\frac{\partial^{2} f_{t-1}\left(\theta_{\left.t-2, \text { Time }_{t-1}, \text { Time }_{t-2}, \gamma, X\right)}\right.}{\partial \theta_{t-2} \partial \text { Time }_{t-2}{ }^{\prime}}>0
$$

Combined with self-productivity, these cross-complementarities (Cunha and Heckman 2007) provide a basis for predicting why some SES gaps in time investments may widen. 
Returning to parental decisions about time investments, consider the case where utility depends solely and linearly on the adult earnings of their children in period $t$, with earnings also being a linear function of human capital. Then parents will choose investments in their children to maximise:

$$
\operatorname{Max} U_{t}=\theta_{t-1},
$$

subject to the relevant budget restriction. In the absence of credit constraints, we would expect parents to be able to borrow against future earnings of their children, and any intergenerational correlation between the human capital of parents and children would operate solely through transmission of initial endowments. Instead if households operate according to a budget constraint dependant on income at the time investment decisions are made (i.e. they are unable to borrow from the adult time period), then the sum of income in both childhood time periods must cover the total cost of the corresponding time investment decisions. 